\title{
In vivo imaging of the voltage-gated potassium channel Kv10.1 utilizing SPECT in combination with radiolabeled antibodies
}

\author{
Doctoral thesis \\ In partial fulfillment of the requirements for the degree \\ "Doctor rerum naturalium (Dr. rer. nat.)" \\ in the Molecular Medicine Study Program \\ at the Georg-August-University Göttingen
}

submitted by

Thomas Krüwel, M.Sc.

born in Hannover

Göttingen, September 2015 
Members of the Thesis Committee:

Supervisor

Prof. Dr. Frauke Alves

Department of Haematology and Medical Oncology

Institute of Interventional and Diagnostic Radiology

University Medical Center Göttingen, Göttingen

and

Department of Molecular Biology of Neuronal Systems

Max-Planck Institute for Experimental Medicine, Göttingen

Second member of the Thesis Committee

Prof. Dr. Walter Stühmer

Department of Molecular Biology of Neuronal Systems

Max-Planck Institute for Experimental Medicine, Göttingen

Third member of the Thesis Committee

Senior-Prof. Dr. Eberhard Fuchs

German Primate Center, Göttingen

Date of Disputation: 


\section{AFFIDAVIT}

Here I declare that my doctoral thesis entitled "In vivo imaging of the voltage-gated potassium channel Kv10.1 utilizing SPECT in combination with radiolabeled antibodies" has been written independently with no other sources and aids than quoted.

Thomas Krüwel

Göttingen, September 2015 


\section{Publications}

Krüwel T., Nevoltris D., Bode J., Dullin C., Baty D., Chames P., Alves F.: In vivo detection of small tumor lesions by multi-pinhole SPECT applying a 99mTc-labeled nanobody targeting the Epidermal Growth Factor Receptor. Scientific Reports (submitted).

Fasshauer M.*, Krüwel T.*, Zapf A., Stahnke V., Rave-Fränk M., Staab W., Sohns J. M., Steinmetz M., Unterberg-Buchwald C., Schuster A., Ritter C., Lotz J.: Absence of DNA double strand breaks in human peripheral blood mononuclear cells after magnetic resonance imaging assessed by $\mathrm{\gamma H} 2 \mathrm{AX}$ flow cytometry. Nature Communications (submitted). * equal contribution

Breckwoldt M., Bode J., Kurz F., Hoffmann A., Ochs K., Ott M., Krüwel T., Deumelandt K., Schwarz D., Fischer M., Helluy X., Milford D., Kirschbaum K., Solecki G., Chiblak S., Abdollahi A., Winkler F., Wick W., Platten M., Heiland S., Bendszus M., Tews B.: Correlated MR imaging and ultramicroscopy (MR-UM) is a tool kit to assess the dynamics of glioma angiogenesis. elife (under revision).

Menke J., Krüwel T., Dullin C. (2015): Fluorochrome-labeled monoclonal antibody with characteristic M-shaped spectral peak for optical imaging: Dual-labeling versus mixture of fluorochromes. Chinese Optics Letters 13 (5): 051701.

Kruewel T., Schenone S., Radi M., Maga G., Rohrbeck A., Botta M., Borlak J. (2010) Molecular Characterization of c-Abl/c-Src Kinase Inhibitors Targeted against Murine Tumour Progenitor Cells that Express Stem Cell Markers. PLoS ONE 5 (11): e14143. 


\section{Table of contents}

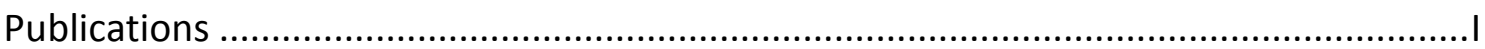

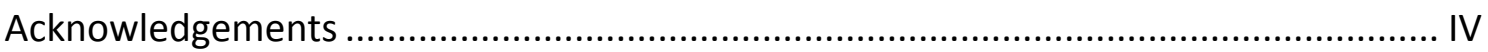

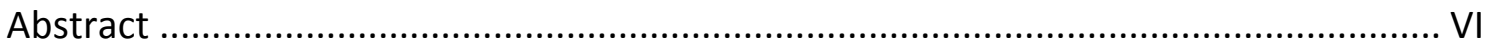

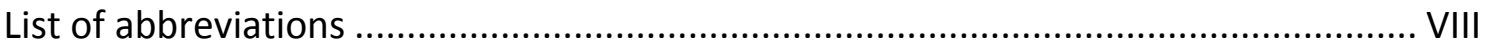

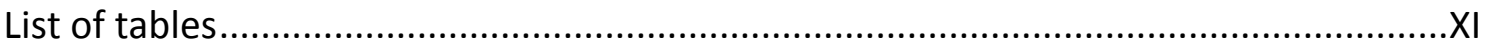

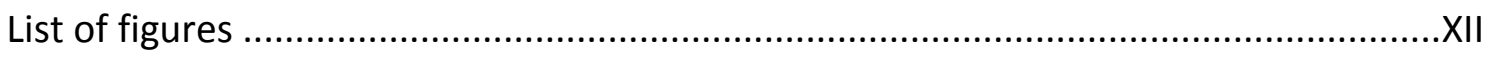

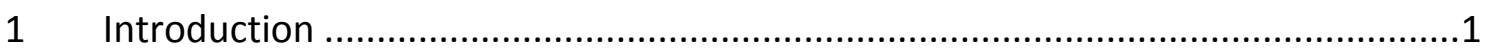

1.1 The voltage-gated potassium channel Kv10.1 .......................................2

$1.2 \quad$ Epidermal growth factor receptor (EGFR) .......................................

$1.3 \quad$ Nanobodies as imaging probes.........................................................6

1.4 Radiolabeling of biomolecules with technetium-99m.............................

1.5 Three dimensional nuclear imaging modalities PET and SPECT .............10

1.6 The orthotopic tumor mouse model ..................................................14

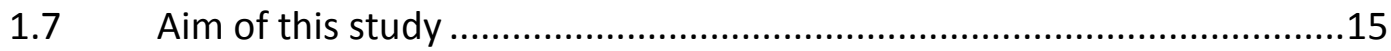

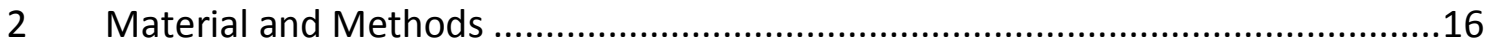

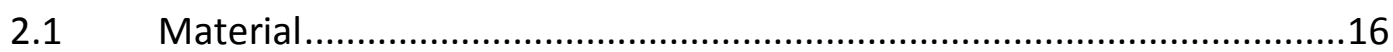

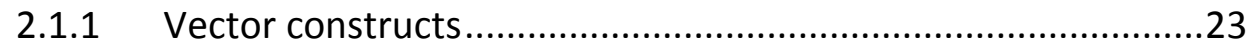

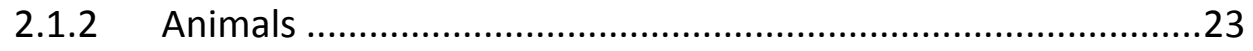

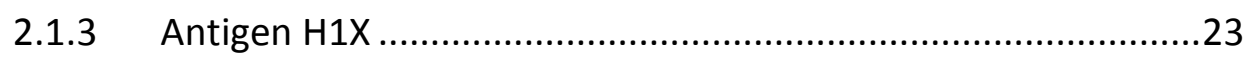

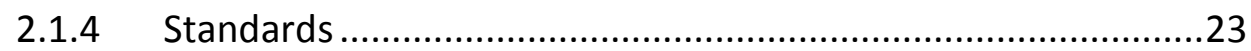

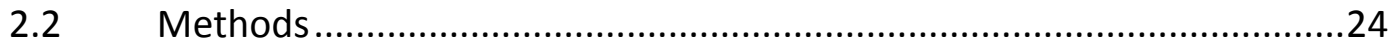

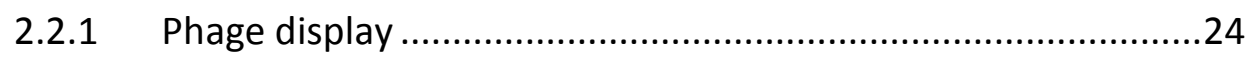

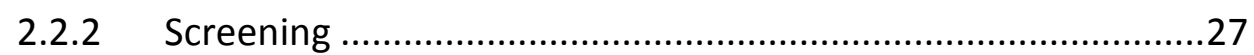

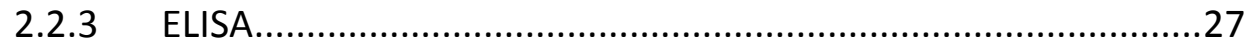

2.2.4 Production of chemically competent E. coli BL21(DE) cells ......27

2.2.5 Plasmid isolation .................................................................28

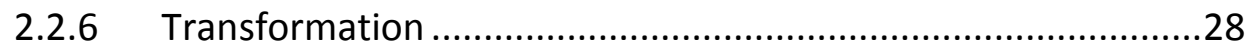

2.2.7 Protein expression and purification .......................................28

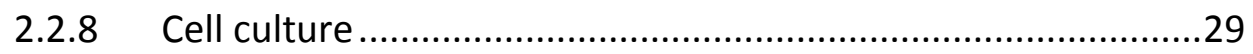

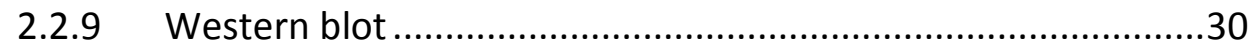

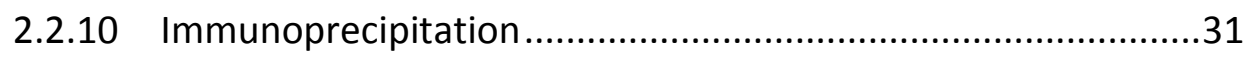

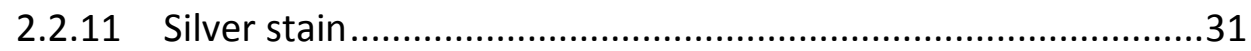

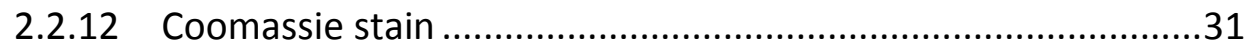

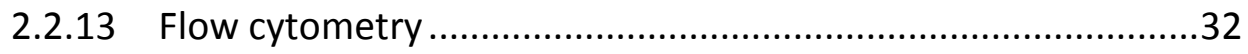


2.2.14 Surface plasmon resonance................................................32

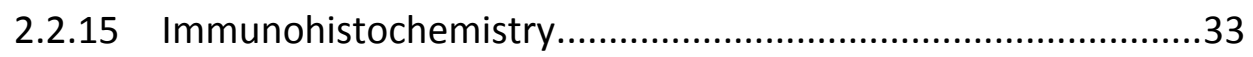

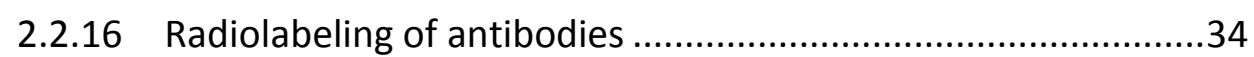

2.2.17 Radiolabeling of nanobodies ................................................34

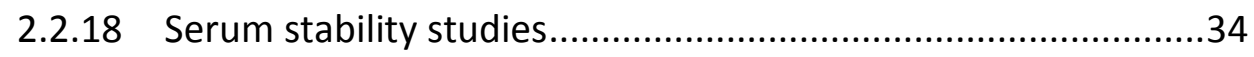

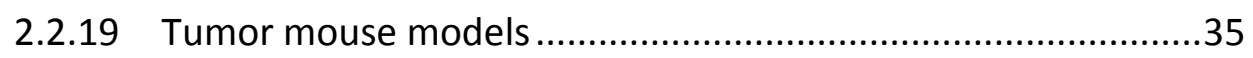

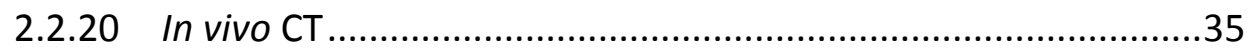

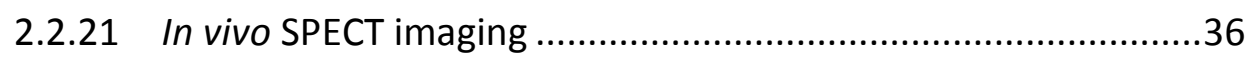

2.2.22 Ex vivo biodistribution analysis............................................36

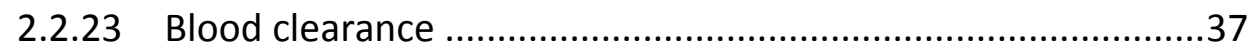

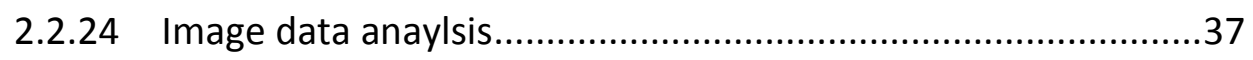

2.2.25 Statistical analyis.............................................................38

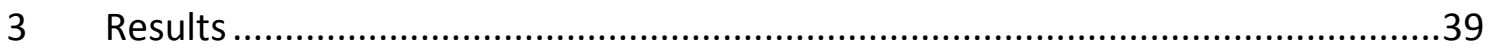

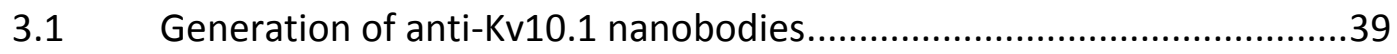

3.1.1 Selection of ten anti-Kv10.1 nanobodies................................39

3.1.2 The anti-Kv10.1 nanobodies detect Kv10.1 ............................45

3.2 Establishment of the SPECT infrastructure .........................................53

3.3 Validation of EGFR expressing tumor models for SPECT imaging ............55

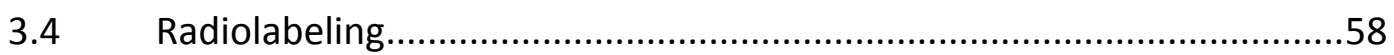

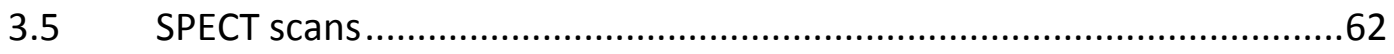

3.5.1 Initial SPECT scans.............................................................62

3.5.2 Tumor visualization with ${ }^{99 \mathrm{~m}}$ Tc-Cetuximab .............................65

3.5.3 Tumor visualization with anti EGFR nanobody ${ }^{99 \mathrm{~m}} \mathrm{Tc}-\mathrm{D} 10 \ldots \ldots . .73$

3.5.4 Visualization of very small tumor lesions ...............................77

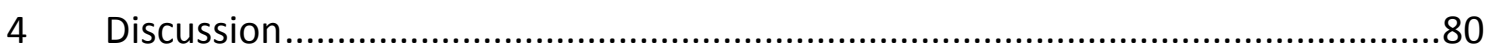

4.1 Generation of specific anti-Kv10.1 nanobodies C4 and D9 ..................80

4.2 Is Kv10.1 a suitable target for molecular imaging? ................................82

4.3 Nanobodies as versatile tools in biology and medicine ..........................83

4.4 Nanobodies and antibodies as tools for molecular imaging ...................84

4.4.1 Tumor visualization with ${ }^{99 \mathrm{~m}}$ Tc-Cetuximab by SPECT ...............84

4.4.2 Application of anti-EGFR nanobody ${ }^{99 \mathrm{~m}}$ Tc-D10 .....................87

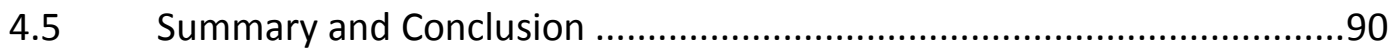

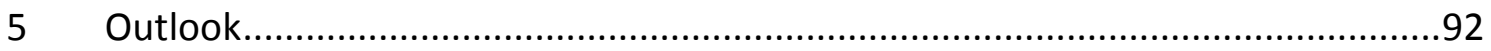

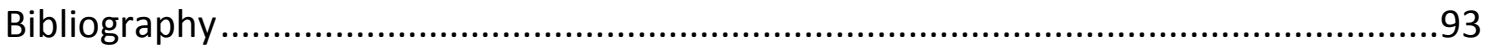

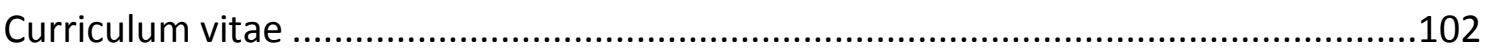




\section{Acknowledgements}

During the last four years I was accompanied by many people who supported me in the completion of my doctoral thesis.

Foremost, I want to express my gratitude to my supervisor and head of the Thesis Committee Prof. Frauke Alves for giving me the opportunity to work in the interdisciplinary and fast growing field of molecular imaging. I deeply acknowledge her for giving me the freedom of doing my work independently, for her inspiring and encouraging attitude and, of course, her critical corrections of my manuscripts accompanied with controversial discussions shaping not only my scientific but also my personal profile. Thank you Frauke.

I also want to thank my boss Prof. Joachim Lotz for providing me with long and worryfree contracts, a rarity in science. I greatly acknowledge him for giving me the opportunity to attend conferences and workshops all over the world, to travel to collaborators and thus to improve my professional profile.

Furthermore, I want to thank the members of my Thesis Committee Prof. Walter Stühmer and Prof. Eberhard Fuchs for the fruitful discussions during my Thesis Committee meetings and for their great support whenever needed. I am very grateful to Walter, for integrating me in his group at the MPI for Experimental Medicine, hosting me and funding a great part of my work.

I thank the members of my extended Thesis Committee, Prof. Luis Pardo, Prof. Hubertus Jarry and Prof. Dörthe Katschinski for their time to read my thesis and attend my defense.

I thank Prof. Johannes Meller and the staff of the Dept. of Nuclear Medicine, especially Jack Schmiereck for hosting me and providing radioactivity.

A big thanks to my collaborators Dr. Patrick Chames and Dr. Daniel Baty for hosting me during my research stay in Marseille in 2014 and providing me with the information to identify the anti-Kv10.1 nanobodies. The fruitful discussions and the supply of the antiEGFR nanobody led to a great outcome. 
I want to express my gratitude and my deepest respect to Christian Dullin, who initiated my position in Göttingen in 2011. Without his help regarding the construction and completion of the imaging infrastructure, necessary for starting my work, I would not be able to complete my project. Furthermore, he programmed and customized his software Scry to my needs to be able to analyze the SPECT and CT data. Again, thank you Christian, I could not get this far without your help.

The support of the workshop of the MPI, especially Robert Otremba was invaluable for my work. Coming with a blueprint and leaving with a $100 \%$ functioning tool, customized for my needs beats every 3D printer.

Also, I want to express my gratitude to the members of the MPI and UMG lab, especially Prof. Luis Pardo, Dr. Sabine Martin, Dr. Franziska Hartung, PD Dr. Olaf Jahn, Thomas Liepold, Lars van Werven, Bärbel Heidrich, Hanna Puchala, Sarah Greco, Mercedes Martin-Ortega, Roswitha Streich, Bettina Jeep, Sabine Wolfgramm and Julia Schirmer for fruitful discussions, support and assistance if needed.

Above all I need to thank my family and friends. Even though we are dispersed over the whole country, from Hannover to Berlin,. Brunswick to Heidelberg and Munich to Regensburg I am glad to have so many awesome people around me. Thank you so much for supporting me all the time and for giving me the opportunity to back away from work in order to load new energy and positive attitude.

Finally, I bow to Julia, who managed to keep my spirits up through the last years and the final stages of the thesis. I am grateful for all your support, time, help, understanding and simply everything. Thank you. 


\section{Abstract}

The detection of tumors in an early phase of tumor development is an important achievement to improve the overall prognosis of the patient. Besides accurate information of tumor load and spread, the retrieval of the expression of biomarkers on the tumor cell surface at the earliest time point is a prerequisite for a successful targeted therapeutic approach. In order to acquire information on expression of tumor associated proteins in vivo, functional imaging with specific probes targeting tumor biomarkers such as human voltage-gated potassium channel Kv10.1 or epidermal growth factor receptor 1 (EGFR) is a promising approach. In this study, 10 novel antiKv10.1 nanobodies were generated by phage display and characterized in vitro, in order to be able to visualize tumor lesions in vivo in future by applying multi-pinhole SPECT targeting Kv10.1. Molecular characterization of the binding properties of the anti-Kv10.1 nanobodies using ELISA, immunoprecipitation, Western blotting, flow cytometry and surface plasmon resonance identified two promising clones, C4 and D9, specifically detecting Kv10.1 transfected HEK cells and the fusion protein H1X.

Since the SPECT system was not used for imaging of small animals before, the system and the imaging procedure was first set up and validated by using the clinically

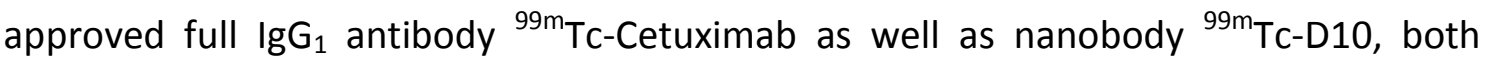
targeting EGFR in human MDA-MB-231 and MDA-MB-468 mammary carcinoma as well as in A431 epidermoid tumor bearing mice.

The anti-EGFR nanobody ${ }^{99 \mathrm{~m}} \mathrm{Tc}-\mathrm{D} 10$ was efficiently labeled with $\left[{ }^{99 \mathrm{~m}} \mathrm{Tc}(\mathrm{CO})_{3}\left(\mathrm{OH}_{2}\right)_{3}\right]^{+}$ yielding a specific activity of $183 \mathrm{MBq} / \mathrm{nmol} \pm 35$ and a radiochemical purity of $97.7 \% \pm 1.2$ whereas the Hynic-derivatized antibody ${ }^{99 \mathrm{~m}} \mathrm{Tc}$-Cetuximab yielded a specific activity of $1700 \mathrm{MBq} / \mathrm{nmol} \pm 105$ and a purity of $97.3 \% \pm 0.4$.

In vivo, MDA-MB-468 and MDA-MB-231 mammary tumors were visualized by SPECT applying 98 pmol of the anti-EGFR antibody ${ }^{99 \mathrm{~m}} \mathrm{Tc}$-Cetuximab with tumor uptakes of $5.49 \% \mathrm{ID} / \mathrm{cm}^{3} \pm 2.2$ and $2.13 \% \mathrm{ID} / \mathrm{cm}^{3} \pm 0.37$, respectively, $24 \mathrm{~h}$ post i.v. injection. Mice bearing MDA-MB-468 tumors that received the isotype control ${ }^{99 m} \mathrm{Tc}-\lg G_{1}$ antibody (98 pmol) showed a significant uptake of $2.1 \% \mathrm{ID} / \mathrm{cm}^{3} \pm 0.1$ to the tumor after $24 \mathrm{~h}$ 
post i.v. injection proving a high unspecific tumor uptake of the antibody. Biodistribution analysis of mice receiving ${ }^{99 \mathrm{~m}} \mathrm{Tc}$-Cetuximab revealed high tumor uptakes of $14.6 \% \mathrm{ID} / \mathrm{g} \pm 6.89$ and $6.19 \% \mathrm{ID} / \mathrm{g} \pm 2.71$ for MDA-MB-468 and MDA-MB231 tumors, respectively, compared to a remaining activity in the blood of approx. $5.5 \% \mathrm{ID} / \mathrm{g}$ in both cohorts resulting in tumor-to-blood ratios of 3.1 and 1.2, respectively. Since ${ }^{99 \mathrm{~m}} \mathrm{Tc}$-Cetuximab is cleared via hepatic excretion from the body, a high liver uptake of approx. 20\% ID/g was determined.

Visualization of very small EGFR positive epidermoid A431 and mammary MDA-MB468 tumors with nanobody ${ }^{99 \mathrm{~m}} \mathrm{TC}-\mathrm{D} 10$ by SPECT imaging already 45 min post i.v. administration resulted in tumor uptakes of $1.0 \% \mathrm{ID} / \mathrm{cm}^{3} \pm 0.6$ and $0.6 \% \mathrm{ID} / \mathrm{cm}^{3} \pm 0.2$, respectively, with excellent in vivo contrast and ex vivo tumor to blood and tissue ratios due to the fast blood clearance with a serum half-life of $4.9 \mathrm{~min}$. The use of control nanobody ${ }^{99 \mathrm{~m}} \mathrm{Tc}-\mathrm{F} 5$ showed no significant tumor uptake. No accumulation of ${ }^{99 m}$ Tc-D10 was observed in MDA-MB-231 tumors characterized by a very low EGFR expression. Biodistribution analysis of ${ }^{99 \mathrm{~m}} \mathrm{Tc}-\mathrm{D} 10$ revealed $\mathrm{A} 431$ and MDA-MB-468 tumor uptakes of $2.3 \% \mathrm{ID} / \mathrm{g} \pm 0.7$ and $1.3 \% \mathrm{ID} / \mathrm{g} \pm 0.3$, respectively, compared to a remaining activity in the blood of approx. $0.2 \% \mathrm{ID} / \mathrm{g}$. This resulted in tumor-to-blood ratios of 12.1 and 5.4 for A431 and MDA-MB-468 tumors, respectively. Since the nanobody ${ }^{99 \mathrm{~m}} \mathrm{Tc}-\mathrm{D} 10$ is cleared via renal excretion from the body, high uptake was determined in the kidneys and the urine $(214 \pm 30 \% \mathrm{ID} / \mathrm{g}$ and $69 \pm 31 \% \mathrm{ID} / \mathrm{g}$, respectively).

The here presented specific and high contrast in vivo visualization of small human tumors overexpressing EGFR by preclinical multi-pinhole SPECT already 45 min after administration of anti-EGFR nanobody ${ }^{99 \mathrm{~m}} \mathrm{Tc}-\mathrm{D} 10$ provides the basis for a possible future in vivo use of anti-Kv10.1 nanobodies for tumor visualization by SPECT. 


\section{List of abbreviations}

\begin{tabular}{|c|c|}
\hline $6 x \mathrm{His}$ & Hexahistidine tag \\
\hline$\beta^{+}$ & Positron decay; positron \\
\hline$\beta^{-}$ & Beta decay; electron \\
\hline$\gamma$ & Gamma radiation; photon \\
\hline a & annum (year) \\
\hline $\mathrm{Ab}$ & Antibody \\
\hline ABTS & 2,2'-azino-bis(3-ethylbenzothiazoline-6-sulphonic acid) \\
\hline AF488 & AlexaFluor 488 dye \\
\hline approx. & approximately \\
\hline BCA & Bicinchoninic acid \\
\hline BGO & Bismuth germanate \\
\hline BSA & Bovine serum albumin \\
\hline $\mathrm{Bq}$ & Becquerel \\
\hline cDNA & Complementary deoxyribonucleic acid \\
\hline CDR & Complementary determining region \\
\hline CEA & Carcinoembryonic antigen \\
\hline CFU & Colony forming unit \\
\hline $\mathrm{CHO}$ & Chinese hamster ovary \\
\hline CMV & Cytomegalovirus \\
\hline CT & Computed Tomography \\
\hline CTTN & Cortactin \\
\hline $\mathrm{Da}$ & Dalton \\
\hline DAB & 3,3'-Diaminobenzidine \\
\hline DMEM & Dulbecco's Modified Eagle Medium \\
\hline DMF & Dimethylformamide \\
\hline DMSO & Dimethy sulfoxide \\
\hline DNA & Deoxyribonucleic acid \\
\hline E. coli & Escherichia coli \\
\hline Eag1 & Ether à go-go voltage-gated channel (Kv10.1) \\
\hline ECL & Enhanced chemiluminescence \\
\hline EDDA & Ethylenediamine- $N, N$ '-diacetic acid \\
\hline EDTA & Ethylenediaminetetraacetic acid \\
\hline EGF & Epidermal growth factor \\
\hline EGFR & Epidermal growth factor receptor \\
\hline ELISA & Enzyme linked immunosorbent assay \\
\hline FAK & Focal adhesion kinase \\
\hline FcRn & Neonatal Fc receptor \\
\hline FCS & Fetal calf serum \\
\hline FDG & ${ }^{18}$ F-fluordeoxyglucose \\
\hline FOV & Field of view \\
\hline FR & Framework \\
\hline GFP & Green fluorescent protein \\
\hline $\mathrm{h}$ & Hour \\
\hline $\mathrm{HcAb}$ & Heavy-chain antibody \\
\hline
\end{tabular}




\begin{tabular}{|c|c|}
\hline HEK & Human embryonic kidney 239 cell line \\
\hline HIF-1 & Hypoxia-inducible factor \\
\hline HRP & Horseradish peroxidase \\
\hline HyNic & 6-hydrazinonicotinamide \\
\hline IMAC & Immobilized metal affinity chromatography \\
\hline IMGT & International immunogenetics information system \\
\hline lgG & Immunoglobulin G \\
\hline IHC & Immunohistochemistry \\
\hline IP & Immunoprecipitation \\
\hline IRES & Internal ribosomal entry site \\
\hline ITLC & Instant thin layer chromatography \\
\hline KCHN1 & Kv10.1 gene \\
\hline Kv10.1 & Voltage-gated potassium channel Kv10.1 (Eag1) \\
\hline l & Liter \\
\hline i.p. & Intraperitoneal \\
\hline i.v. & Intravenous \\
\hline LB & Luria broth \\
\hline LDS & Lithium dodecyl sulfate \\
\hline M & Molar \\
\hline $\mathrm{mAb}$ & Monoclonal antibody \\
\hline MALDI & Matrix-assisted Laser Desorption / Ionization \\
\hline MFI & Mean fluorescence intensity \\
\hline $\min$ & Minute \\
\hline MOPS & 3-(N-morpholino)propanesulfonic acid \\
\hline MS & Mass spectrometry \\
\hline mRNA & Messenger ribonucleic acid \\
\hline MWCO & Molecular weight cut off \\
\hline NOD & Non-obese diabetic \\
\hline OD & Optical density \\
\hline OSEM & Ordered subset expectation maximization \\
\hline $\mathrm{pAb}$ & Polyclonal antibody \\
\hline PAGE & Poly acrylamide gel electorphoresis \\
\hline PBS & Phosphate buffered saline \\
\hline PBST & Phosphate-buffered saline containing Tween 20 \\
\hline PE & R-Phycoerythrin \\
\hline PES & Polyethersulfone \\
\hline PET & Positron emission tomography \\
\hline PFA & Paraformaldehyde \\
\hline PSMA & Prostate specific membrane antigen \\
\hline Rab5 & Rabaptin-5 \\
\hline REF & Reference structure \\
\hline RIPA & Radioimmunoprecipitation assay \\
\hline rpm & Revolutions per minute \\
\hline RT & Room temperature \\
\hline s.c. & Subcutaneous \\
\hline SCID & Severe combined immune deficient \\
\hline SD & Standard deviation \\
\hline SDS & Sodium dodecyl sulfate \\
\hline
\end{tabular}




$\begin{array}{ll}\text { Sec } & \text { Second } \\ \text { SG } & \text { Silica gel } \\ \text { SHNH } & \text { Succinimidyl 6-hydraziniumnicotinate hydrochloride } \\ \text { SPECT } & \text { Single photon emission computed tomography } \\ \text { SPIM } & \text { Single plane illumination microscopy } \\ \text { SPR } & \text { Surface plasmon resonance } \\ \text { SV40 } & \text { Simian vacuolating virus 40 } \\ \text { TBS } & \text { Tris-buffered saline } \\ \text { TBST } & \text { Tris-buffered saline containing Tween20 } \\ \text { TGF } \alpha & \text { Transforming growth factor- } \alpha \\ \text { TKI } & \text { Tyrosine-kinase inhibitor } \\ \text { TOF } & \text { Time of flight } \\ \text { TRX } & \text { Thioredoxin } \\ \text { U } & \text { Unit } \\ \text { V/v } & \text { Volume by volume } \\ \text { VEGF } & \text { Vascular endothelia growth factor } \\ \text { VHH } & \text { Variable fragment of a heavy chain antibody } \\ \text { W/v } & \text { Weight by volume } \\ \text { WHO } & \text { World Health Organization } \\ \text { qRT-PCR } & \text { Quantitative Real-time polymerase chain reaction } \\ \varnothing & \text { Empty plasmid }\end{array}$




\section{List of tables}

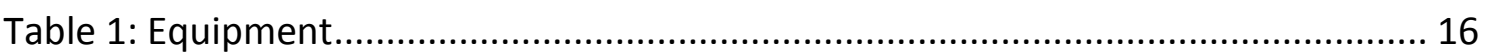

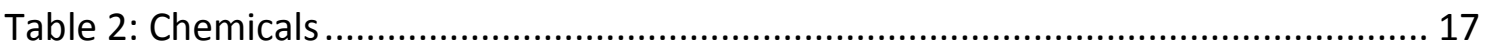

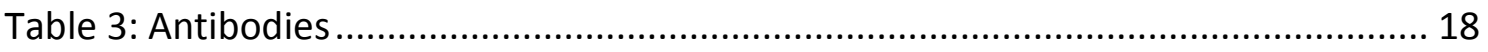

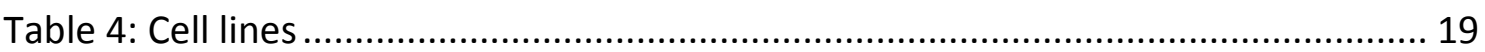

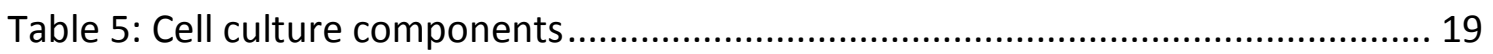

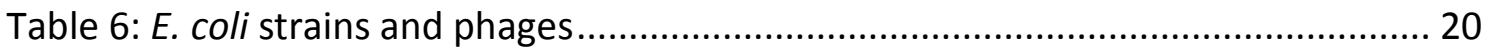

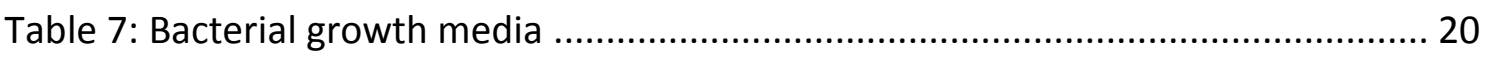

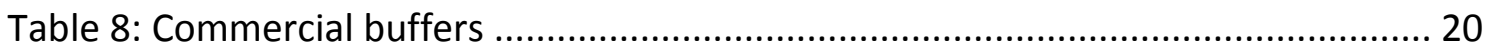

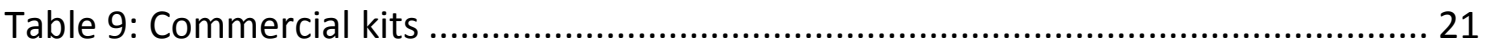

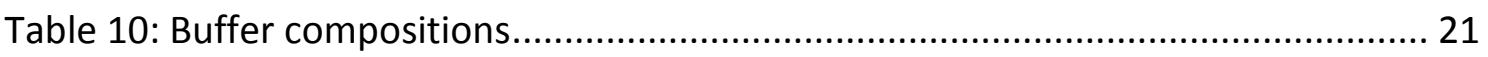

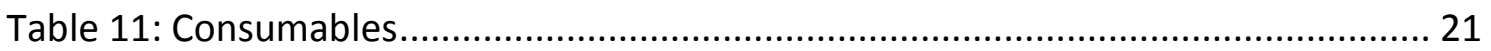

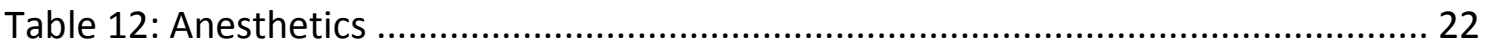

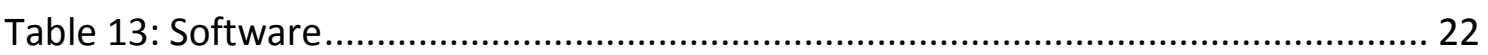

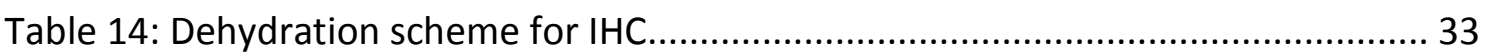

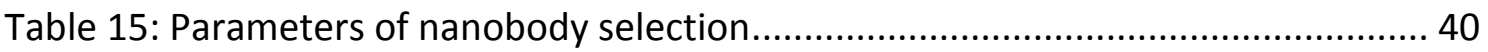

Table 16: Identification of nine unique anti-Kv10.1 nanobodies by sequencing........... 42

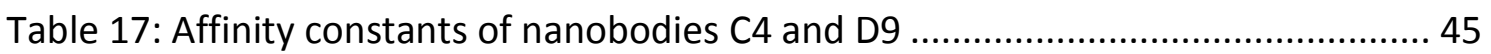

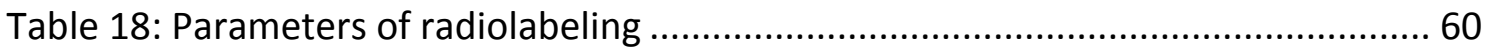

Table 19: In vivo determined tumor and tissue uptake of ${ }^{99 \mathrm{~m}} \mathrm{Tc}$-Cetuximab ................... 69

Table 20: Ex vivo determined tumor and tissue uptake of ${ }^{99 \mathrm{~m}}$ Tc-Cetuximab ................ 72

Table 21: Tumor and tissue uptake of ${ }^{99 \mathrm{~m}} \mathrm{Tc}-\mathrm{D} 10$ and ${ }^{99 \mathrm{~m}} \mathrm{Tc}$-Cetuximab........................ 76 


\section{List of figures}

Figure 1: Schematic structure of voltage-gated potassium channels ............................. 2

Figure 2: Schematic structure of a EGFR molecule in the cellular membrane................. 5

Figure 3: Schematic drawing of a heavy chain antibody and a nanobody..................... 7

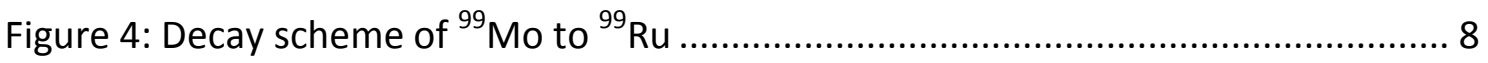

Figure 5: Molecular structure of a HyNic-modified biomolecule (R) ............................. 9

Figure 6: Monodentate and bidentate binding of HyNic to ${ }^{99 \mathrm{~m}} \mathrm{Tc}$............................... 9

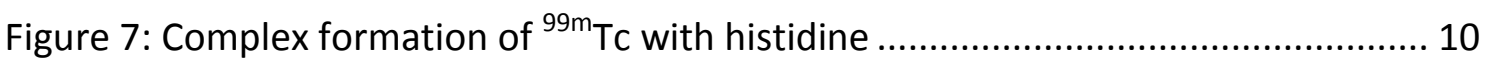

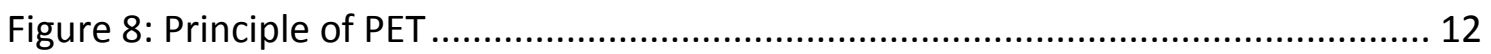

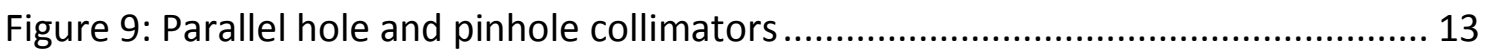

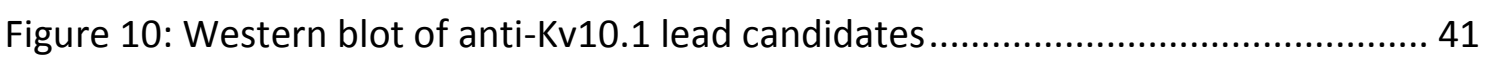

Figure 11: Binding capacities of anti-Kv10.1 nanobodies ........................................... 43

Figure 12: Sensograms of anti-Kv10.1 nanobodies C4 and D9.................................. 44

Figure 13: ELISA of anti-Kv10.1 nanobody lead candidates ........................................ 45

Figure 14: Specific detection of blotted H1X by nanobody C4 ...................................... 46

Figure 15: Detection of Kv10.1 by immunoprecipitation ........................................... 48

Figure 16: Silver stained enrichment of Kv10.1 by the use of nanobody D9 ................. 50

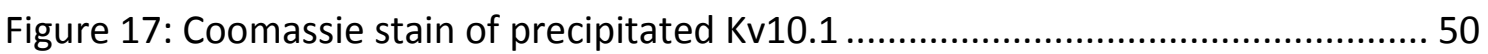

Figure 18: Flow cytometry of Kv10.1 transfected cells with nanobodies C4 and D9.... 52

Figure 19: Jaszczak-Phantom scans using different collimators .................................. 54

Figure 20: Validation of EGFR expression by Western blotting .................................... 55

Figure 21: Validation of EGFR expression by flow cytometry ..................................... 56

Figure 22: Validation of EGFR expression levels in tumors by IHC.............................. 58

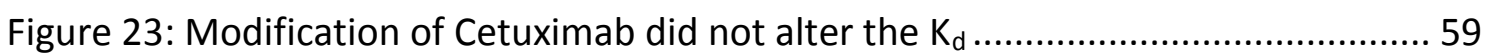

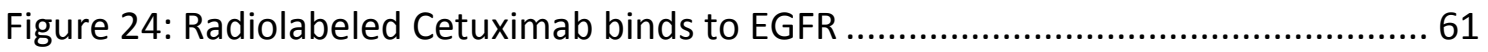

Figure 25: Assessment of binding capacities of radiolabeled nanobodies ..................... 62

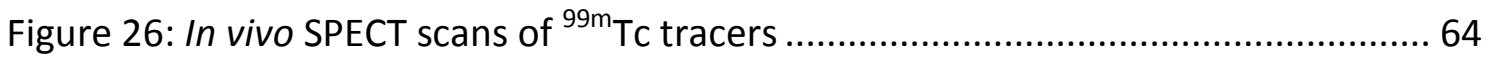

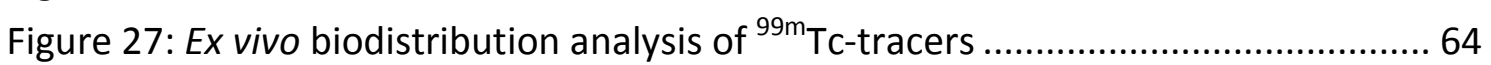

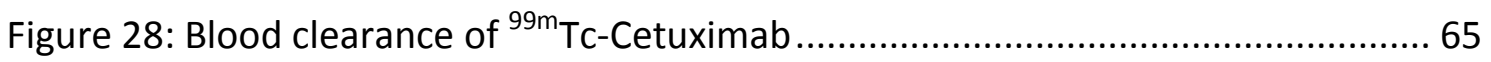

Figure 29: Uptake of ${ }^{99 \mathrm{~m}} \mathrm{Tc}$-Cetuximab to MDA-MB-468 tumors was specific.................67 67

Figure 30: Tumor uptake of ${ }^{99 \mathrm{~m}}$ Tc-Cetuximab was dependent on EGFR expression ..... 70

Figure 31: Ex vivo biodistribution analysis of ${ }^{99 \mathrm{~m}} \mathrm{Tc}$-Cetuximab in tumor bearing mice 71

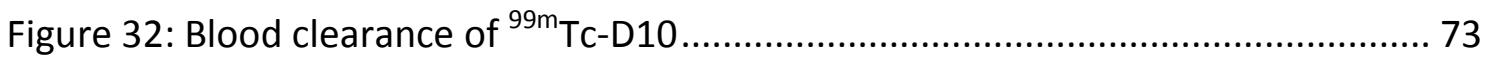

Figure 33: Tumor uptake of ${ }^{99 \mathrm{~m}} \mathrm{Tc}-\mathrm{D} 10$ was dependent on EGFR expression ................ 74

Figure 34: Tumor visualization of mammary MDA-MB-468 and MDA-MB-231 tumors 75

Figure 35: Uptake of anti-EGFR nanobody ${ }^{99 \mathrm{~m}} \mathrm{Tc}-\mathrm{D} 10$ in A431 tumors ............................ 78

Figure 36: In vivo tumor visualization of small human A431 tumors with ${ }^{99 \mathrm{~m}} \mathrm{TC}-\mathrm{D} 10$... 79 


\section{Introduction}

Cancers account for more than 8.2 million death cases and about 12 million new cases in 2012 being the leading cause of morbidity and mortality worldwide. Among women, breast cancer has the highest mortality with more than 500,000 death cases in 2012 [1]. According to the World Health Organization (WHO), the only way to reduce cancer mortality is an early detection and treatment of new cases [1]. Especially for breast cancer, clinical breast exams, ultrasound and mammography are provided as screening methods. If abnormalities were detected during the screening procedure that might indicate a hematological malignancy, biopsies are taken and histological analysis assesses morphological changes in tissue and expression of specific tumor markers. Once a tumor lesion was diagnosed, the stage of the disease has to be determined to plan a suitable treatment regimen involving surgical resection and chemotherapy. A widely used method for tumor staging is the application of ${ }^{18} \mathrm{~F}$-fluordeoxyglucose (FDG) with a combined positron emission tomography (PET) and computed tomography (CT) scan $[2,3]$. FDG is a glucose analog with a radioactive fluorine-18 substituted hydroxyl group at the 2' position in the glucose molecule. It is taken up by glucose transporters to the cells and phosphorylated by hexokinase to FDG-6-phosphate, that cannot be further metabolized. The reverse reaction in the cells to FDG is very slow, except in the cells of the liver, thus trapping and enriching radioactivity particularly in cells with a high metabolic rate such as tumor cells $[2,4]$. However, a high glucose uptake also present in the brain, at inflammatory sites or in brown fat tissue among many others, can lead to false positive signals [2]. Furthermore, the metabolic rate of a tumor is an important factor for tumor staging but reveals no information on the tumor markers expressed on the surface. Besides accurate information of tumor load and spread, the retrieval of the expression of biomarkers on the tumor cell surface at the earliest time point is a prerequisite for a successful targeted therapeutic approach. In order to acquire information on expression of tumor associated proteins in vivo, functional imaging with specific probes, labeled with a radionuclide, can be performed noninvasively by positron emission tomography (PET) or single photon emission computed tomography (SPECT) with high sensitivity. Targeting biomarkers such as human epidermal growth factor receptor 1 (EGFR) or 2 (HER2) that are highly overexpressed 
on tumor cells is a recently applied strategy [5-10]. However, new targets are in need for reliable tumor detection. Recently, ion channels have been discussed as promising targets not only for therapy but also for in vivo imaging approaches in oncology [11, 12].

\subsection{The voltage-gated potassium channel Kv10.1}

The voltage-gated potassium channel Kv10.1 or Ether à go-go voltage-gated channel (Eag1) is a transmembrane protein consisting of six membrane-spanning domains, three cytosolic loops, a pore region and complex intracellular $\mathrm{N}$ - and $\mathrm{C}$-termini (Figure 1A) [13]. The functional potassium channel consists of four $\alpha$-subunits that assemble the pore of the channel (Figure 1B).
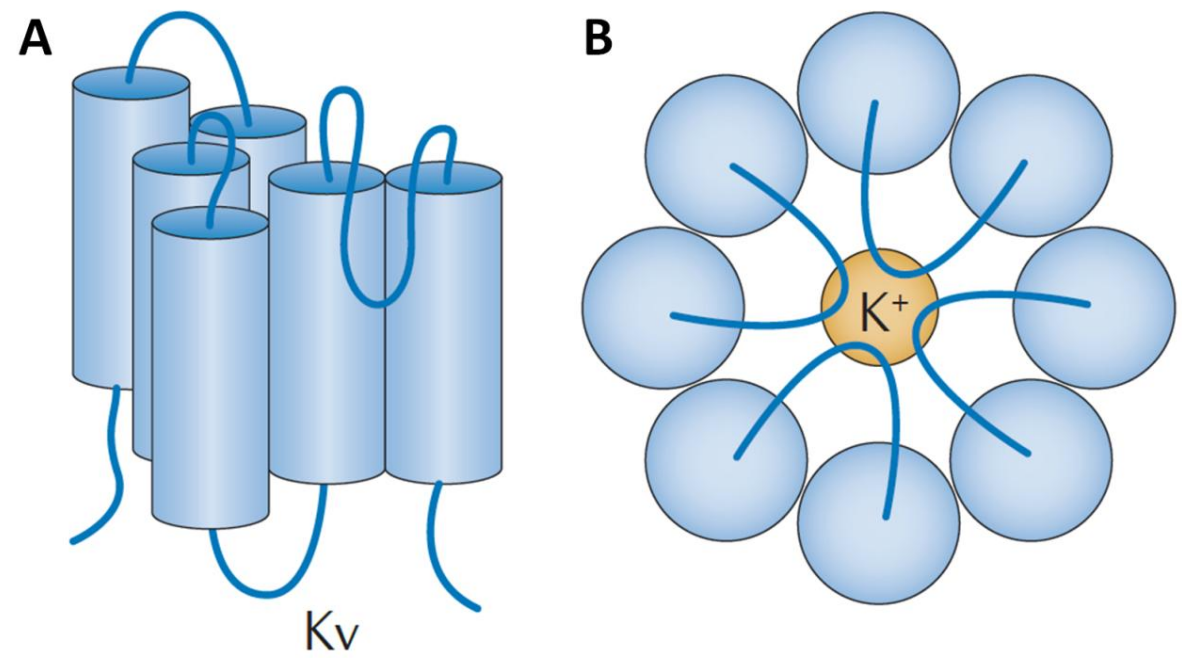

Figure 1: Schematic structure of voltage-gated potassium channels

(A) A lateral view of monomers of a voltage-gated potassium channel (Kv) and (B) a top view of a Kv channel showing the two transmembrane domains of each of the four $\alpha$-subunits and their corresponding pore-forming loops. Image was adapted from [11].

Kv10.1 is predominantly expressed in the brain of humans and rodents, mainly in the olfactory bulb, cerebral cortex, hippocampus, hypothalamus and cerebellum, however the impact for the cells is still unknown [14-17]. Interestingly, no Kv10.1 expression was detected in normal healthy tissue, but was reported for more than a decade, to be expressed on various human tumor entities $[13,18-20]$. More than $70 \%$ out of more 
than thousand investigated tumor samples of different origins were proven Kv10.1 positive, including sarcoma and lymphoma, as well as breast, lung and colon cancer $[13,20]$. In osteosarcoma, the ectopic expression of Kv10.1 is regulated by p38 MAPK/p53 pathway that is triggered by EGFR [21]. Altered expression of p53 interferes with the transcription factor E2F1, that increases Kv10.1 expression and decreases the expression of Kv10.1 suppressor micro RNA mir-34a, resulting in aberrant Kv10.1 expression [22]. The overexpression of Kv10.1 affects proliferation, vascularization and migration of tumor cells through functional interactions with proliferation-related proteins such as the upregulation of hypoxia-inducible factor (HIF-1) leading to an increased secretion of vascular endothelia growth factor (VEGF), thus promoting vascularization [23]. The binding of cortactin (CTTN) and focal adhesion kinase (FAK) to the C-terminal domain of Kv10.1 stabilizes the potassium channel in the membrane and effects adhesion and migration of the tumor cell [24]. These effects are also affected by an altered cell cycle influenced by interaction of Kv10.1 with Rabaptin- 5 (Rab5) or through calcium signaling $[25,26]$. The expression of Kv10.1 correlates with a poor prognosis in various tumor entities like colon, ovarian and head and neck cancer, as well as fibrosarcoma and acute myeloid leukemia [20, 27-30].

A blockade of Kv10.1 with astemizole and imipramine decreased tumor cell proliferation in vitro and in vivo and hold potential as treatment regimen for Kv10.1 positive tumors [20,31,32]. Unfortunately, astemizole and imipramine block Kv11.1 (Eag1-related, HERG) as well and can cause the longQT syndrome, a severe cardiac dysfunction, that led to a withdrawal from the market [12]. Up to now, no selective Kv10.1 blocker exists that could be used for a successful therapy of Kv10.1 positive tumors. 


\subsection{Epidermal growth factor receptor (EGFR)}

The receptor tyrosine kinase EGFR is a transmembrane receptor, that consists of an extracellular, a single transmembrane and an intracellular kinase domain (Figure 2). EGFR is expressed in most epithelial, mesenchymal and neuronal mammalian cells and mediates proliferation and differentiation via various canonical pathways e.g. MAPK (p38), Akt and JNK [33-35]. Binding of a ligand like epidermal growth factor (EGF) or transforming growth factor- $\alpha$ (TGF $\alpha$ ) to the ligand-binding cleft in the extracellular domain induces dimerization of two EGFR monomers and activation of the receptor [36]. The activation involves autophosphorylation of several tyrosine residues in the intracellular C-terminal domain and stimulates the intrinsic tyrosine kinase activity which triggers downstream activation and signaling by several other proteins $[36,37]$.

Due to mutations in many tumors of diverse origin like lung, colon, breast, liver or brain, EGFR is aberrantly expressed or permanently activated, promoting proliferation, survival, migration and angiogenesis leading to uncontrolled growth of tumor cells [38, 39]. The identification of EGFR as an oncogene fostered the development of EGFR targeting antitumor drugs. Two classes of substances are on the market for the treatment of EGFR, tyrosine-kinase inhibitors (TKI) and monoclonal antibodies (mAb).

TKIs are so-called small molecule drugs with a molecular weight below $500 \mathrm{~g} / \mathrm{mol}$ (Da), that bind to the adenosine triphosphate (ATP) binding site on the intracellular Cterminal domain of the EGFR. They prevent the phosphorylation and thereby the activation of the EGFR itself and of downstream signaling pathways [40]. First generation TKIs like Gefitinib, Erlotinib and Lapatinib bind reversibly to the ATP binding site resulting in limited therapeutic outcome as first line treatment [41-43]. A recently approved TKI, Afatinib, binds irreversibly to a thiol group of a cysteine and belongs to the so-called second generation TKIs $[41,44]$. However, the benefit of these drugs to the clinical outcome has not been fully evaluated yet [39]. 


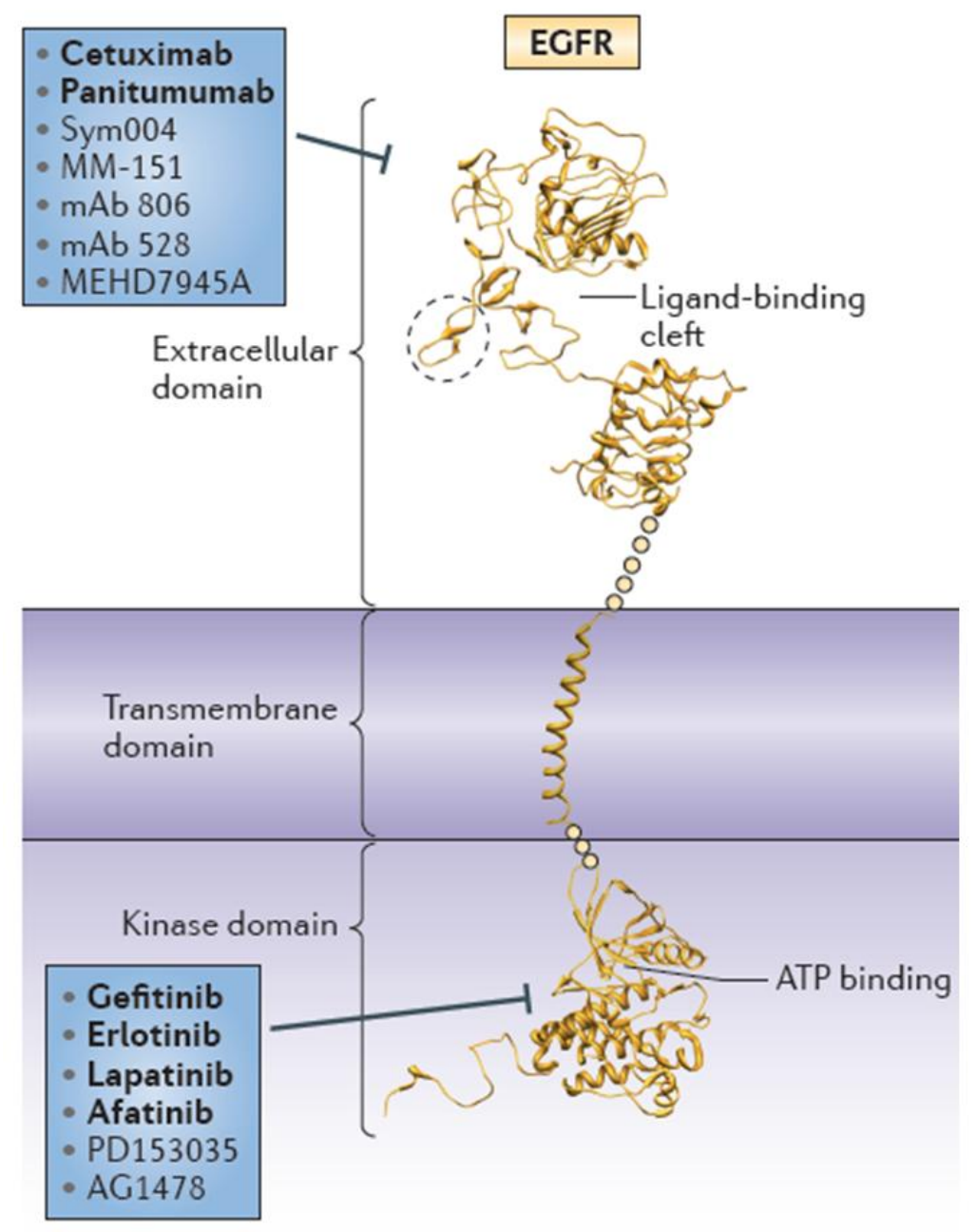

Figure 2: Schematic structure of a EGFR molecule in the cellular membrane

The EGFR consists of an extracellular, a single transmembrane and an intracellular kinase domain. The ligand-binding cleft in the extracellular domain interacts with a number of ligands, including EGF and TGF $\alpha$. In presence of a ligand, dimers form through interactions between the dimerization loop (dashed circle) on each monomer. Dimerization results in the activation of the kinase domain and the initiation of intracellular signaling pathways. Therapeutic antibodies and tyrosine kinase inhibitors (blue boxes) are directed against the ligand-binding cleft in the extracellular domain and to the intracellular kinase domain, respectively. Bold-written drugs have been FDA-approved. Image was adapted from [39].

Compared to TKIs, mAbs like Cetuximab or Panitumumab are huge molecules with a molecular weight of approx. $150 \mathrm{kDa}$, that bind to the ligand-binding cleft on the extracellular domain and prevent the ligands from binding and impede dimerization [45]. Binding of a mAb to EGFR results in a slow internalization by endocytosis of the EGFR-mAb complex to the cell. Following ubiquitination, the internalized vesicle is targeted to the lysosome for lysosomal degradation. Consequently, less EGFR molecules on the cellular surface result in decreased activation of downstream 
signaling [39]. The successful use of mAbs for chemotherapy accompanied with the knowledge of the biodistribution and binding characteristics fostered the assessment of mAbs as carrier molecules for cytotoxic payload or radioactive isotopes. Within the last decade, mAbs in general and Cetuximab in particular, labeled with fluorescent dyes or radioisotopes, were frequently used as probes for non-invasive imaging to detect tumors and to monitor treatment response.

\subsection{Nanobodies as imaging probes}

Targeting of tumor associated antigens for diagnostic approaches allows the assessment of the expression of tumor markers in vivo and is, due to intratumoral heterogeneity, important for clinicians to decide on the best treatment. A requirement for probes to be used for tumor imaging is their fast and specific accumulation in the tumor and as little as possible uptake in healthy tissue, thus generating a high contrast within the tumor shortly after probe administration. In order to achieve a fast removal from the blood pool the ideal imaging probe should be as small as possible [46]. Unlike conventional antibodies, nanobodies, also called single domain antibodies, derived from camelid heavy chain antibodies meet all these requirements with a molecular weight of only $15 \mathrm{kDa}$ and dimensions of $2.5 \times 4 \mathrm{~nm}$ [47] (Figure 3). Due to their small size nanobodies are removed quickly from the blood by renal clearance with half-lives in serum of less than 10 min $[7,8]$. Small-sized proteins are also known to extravasate more easily and show a better tissue penetration compared to larger molecules like full antibodies with a molecular weight of $150 \mathrm{kDa}$ [47]. Due to the lack of the Fc part of an intact immunoglobulin $\mathrm{G}(\operatorname{IgG})$, nanobodies are not suspected to interfere with the immune system $[48,49]$. Furthermore, nanobodies are produced in E.coli, that is considered to be an economic, fast and straightforward expression system with high yields. Nanobodies can easily be modified with various tags e.g. hexahistidine (6xHis), myc or a free cysteine that allow a site-specific labeling for biomedical imaging [50].

Nanobodies have been raised against various tumor markers like EGFR, Her2, carcinoembryonic antigen (CEA), as well as prostate specific membrane antigen (PSMA) and have been applied for preclinical in vivo tumor imaging with different radionuclides for PET and SPECT [7, 9, 51-53]. Recent developments were directed 
towards a therapeutic use of nanobodies either as monomers or conjugates of different nanobodies $[54,55]$. Today, bi- and trivalent as well as bi- and trispecific nanobodies are subjects of various clinical trials for cardiovascular, respiratory, hematological and autoimmune diseases [55].

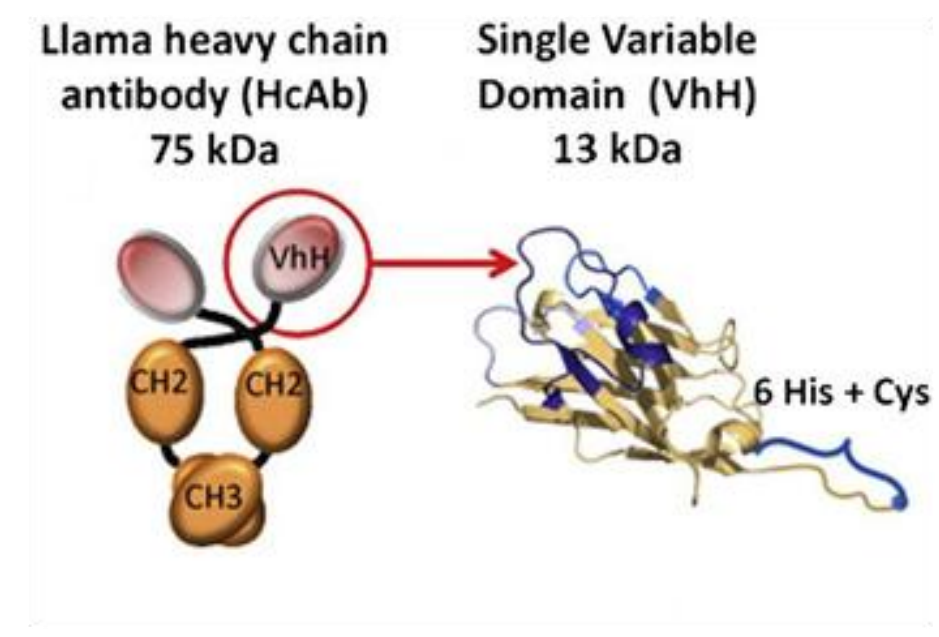

Figure 3: Schematic drawing of a heavy chain antibody and a nanobody

A llama heavy-chain antibody ( $\mathrm{HcAb}$ ) and a representation of a nanobody or single domain antibody (sdAb or $\mathrm{VhH}$ ), the smallest functional llama antibody fragment are shown. The nanobody is engineered with a C-terminal 6xHis tag and a free cysteine (Cys). HcAb and nanobody have a molecular weight of $75 \mathrm{kDa}$ and $15 \mathrm{kDa}$, respectively. Image was adapted from [56].

\subsection{Radiolabeling of biomolecules with technetium-99m}

The radionuclide technetium-99m ( ${ }^{99 m} \mathrm{Tc}, \mathrm{m}=$ metastable) is considered to be the workhorse of nuclear medicine and is applied in more than $80 \%$ of all SPECT imaging examinations, due to its ideal nuclear properties and easy and efficient production in a ${ }^{99} \mathrm{Mo} /{ }^{99 \mathrm{~m}} \mathrm{Tc}$-generator [57-59]. The energy of $140 \mathrm{keV}$ of the emitted gamma radiation is sufficient to penetrate human tissue and allows an external detection of the photons by SPECT scanners [60]. ${ }^{99 \mathrm{~m}} \mathrm{Tc}$ is the daughter nuclide of ${ }^{99} \mathrm{Mo}$ and remains in an excited transition or metastable state for $6.01 \mathrm{~h}$ before it decays to ground state ${ }^{99} \mathrm{Tc}$ and finally to the stable ${ }^{99} \mathrm{Ru}$ (Figure 4)[60,61]. The relatively long half-life is suitable for labeling of proteins like anti- or nanobodies, their application, accumulation to the target tissue and subsequently their detection by in vivo SPECT scans. 


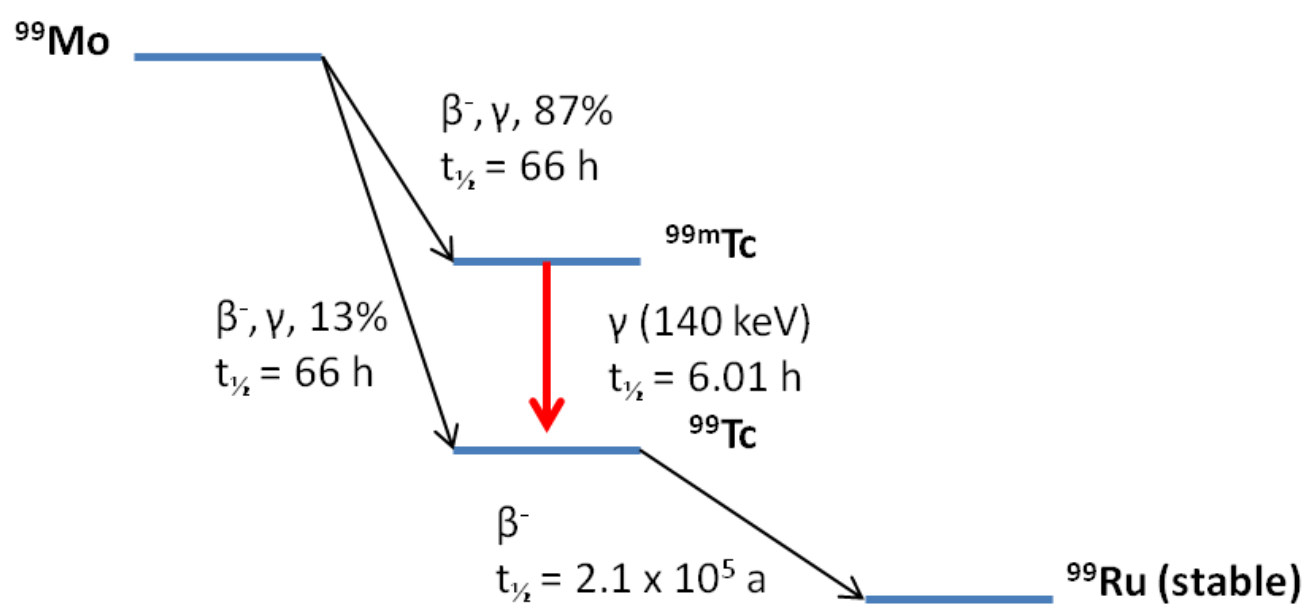

Figure 4: Decay scheme of ${ }^{99} \mathrm{Mo}$ to ${ }^{99} \mathrm{Ru}$

${ }^{99} \mathrm{Mo}$ is generated by neutron activation of ${ }^{98} \mathrm{Mo}$ ( $\mathrm{n}, \mathrm{p}$ reaction) in a high neutron flux reactor. ${ }^{99} \mathrm{Mo}$ is the mother nuclide of ${ }^{99 \mathrm{~m}} \mathrm{Tc}$ with a half-life of $66 \mathrm{~h}$ and decays to ${ }^{99 \mathrm{~m}} \mathrm{Tc}$ by beta $\left(\beta^{-}\right)$decay with a probability of $87 \%$. ${ }^{99 m} \mathrm{Tc}$ remains in an excited transition or metastable state and decays to ground state ${ }^{99} \mathrm{Tc}$ by isomeric transition and emission of gamma radiation (140 keV) with a half-life of $6.01 \mathrm{~h}$. Ground state ${ }^{99}$ Tc decays finally stable ${ }^{99}$ Ru by $\beta^{-}$decay with a half-life of $2.1 \times 10^{5}$ years.

The eluate from the generator, sodium pertechnetate $\left(\mathrm{Na}^{99 \mathrm{~m}} \mathrm{TcO}_{4}\right)$, cannot be used directly for the labeling of proteins because it is chemically inert and does not form stable complexes. The generator-derived $99 \mathrm{mTc}$ has an oxidation state of $+\mathrm{VII}$ and exhibits diverse redox chemistry with various oxidation states ranging from -I to +VII. Therefore, the radiolabeling of antibodies requires a previous modification with 6hydrazinonicotinamide (HyNic) [60-63].

\section{${ }^{99 m}$ Tc-HyNic core}

For the binding to the hydrazine group of HyNic ${ }^{99 \mathrm{~m}} \mathrm{Tc}$ has to be reduced to oxidation state $+l l$ by the use of stannous chloride for example (Figure 5). The condensation reaction forms a chemically robust metal-organohydrazine. HyNic occupies only one or two coordination sites of ${ }^{99 \mathrm{~m}} \mathrm{Tc}$ and therefore requires the use of a coligand like tricine, ethylenediamine- $N, N^{\prime}$-diacetic acid (EDDA) or glucoheptonate to complete a square pyramidal or octahedral complex [64-66]. 
<smiles>[R]NC(=O)c1ccc(NN)nc1</smiles>

Figure 5: Molecular structure of a HyNic-modified biomolecule (R)

High labeling efficiencies can be achieved using the ${ }^{99 \mathrm{~m}} \mathrm{Tc}-\mathrm{HyNic}$ core and pharmacokinetic or hydrophilic modifications can be influenced by the choice of the coligand. The use of tricine as coligand results in the best incorporation and in the highest in vivo stability of the complex $[67,68]$. However, the identity of the ${ }^{99 \mathrm{~m}} \mathrm{Tc}$ species remains unknown and therefore restricted to the use of ${ }^{99 \mathrm{~m}} \mathrm{Tc}-\mathrm{HyNic}$ in clinical applications [64, 66-68] (Figure 6).

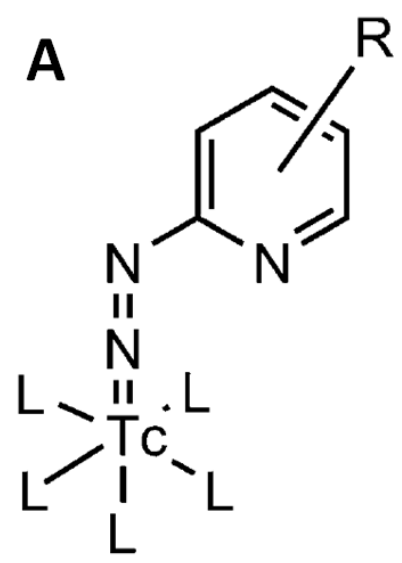

B

Figure 6: Monodentate and bidentate binding of HyNic to ${ }^{99 \mathrm{~m}} \mathrm{Tc}$

(A) Monodentate and (B) bidentate binding mode of HyNic to 99mTc. $\mathrm{R}=$ biomolecule, $\mathrm{L}=$ ligand, tricine. Image was adapted from [67].

$\left[{ }^{99 m} \mathrm{Tc}(\mathrm{CO})_{3}\right]^{+}$core

The discovery of the ${ }^{99 \mathrm{~m}} \mathrm{Tc}$ tricarbonyl $\left.{ }^{99 \mathrm{~m}} \mathrm{Tc}(\mathrm{CO})_{3}\left(\mathrm{OH}_{2}\right)_{3}\right]^{+}$core allowed the preparation of stable organometallic complexes in aqueous solutions, which is favorable for the labeling of biomolecules [69-71] (Figure 7). The complex is formed by reduction of the eluted pertechnetate with sodium borohydride under a carbon monoxide (CO) atmosphere, that is provided by hydrolysis of sodium boranocarbonate $\left(\mathrm{Na}_{2}\left(\mathrm{H}_{3} \mathrm{BCO}_{2}\right)\right)$. The use of this reaction became quite popular due to the supply of a convenient kit 
formulation (Isolink, Mallinckrodt), that was discontinued in 2011. One of the inventors, R. Schibli from Paul-Scherrer-Institute (Villingen, Switzerland) took over the production and supplies now the "CRS Kit for tricarbonyl".

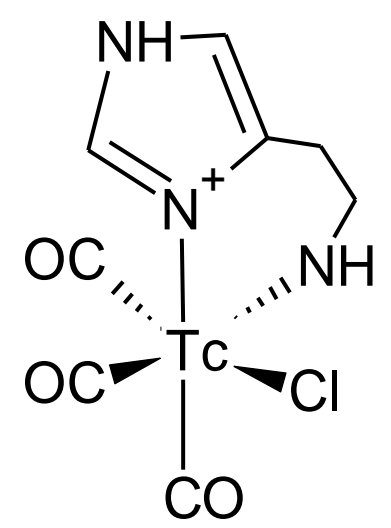

Figure 7: Complex formation of ${ }^{99 \mathrm{~m}} \mathrm{Tc}$ with histidine

For the labeling of biomolecules, the three labile water molecules are easily substituted with $\mathrm{S}, \mathrm{O}$ or $\mathrm{N}$ atoms of various chelating ligands. The amino acid histidine has ideal characteristics for an easy and stable complexation of the tricarbonyl core. Thus, a $6 \mathrm{xHis}$ tag as provided on nanobodies enables high labeling efficiencies and therefore the in vivo application and detection by SPECT [64, 66, 69-72].

\subsection{Three dimensional nuclear imaging modalities PET and SPECT}

PET and SPECT are two cross-sectional imaging modalities in the field of nuclear medicine and were developed in the 1960s and 1970s [73]. Both modalities belong to the area of functional imaging since molecular interactions can be visualized after application of a tracer labeled with a radionuclide. A tracer can be a protein, small molecules or even an unconjugated radionuclide that accumulates in malignant tissues after intravenous (i.v.) injection. The superior sensitivity of PET and SPECT allows a detection of these radionuclides in concentrations of $10^{-6}$ to $10^{-9} \mathrm{M}$ compared to $10^{-3} \mathrm{M}$ of contrast agents afforded for $\mathrm{CT}$ or $\mathrm{MRI}$ examinations $[61,62,66]$. The application of radioactive tracers to the body as well as the exposure to ionizing radiation should always be carefully considered to the expected outcome of the examination. However, due to the very small amounts of radioactivity, the acquired 
effective dose is approx. $7 \mathrm{mSv}$ in a standard FDG-PET scan with an activity of $300 \mathrm{MBq}$, that corresponds to the dose acquired with one chest CT [2]. Except from being tomographic modalities, PET and SPECT require different radionuclides and have distinct differences in the detection thereof.

PET

PET is the most widely applied modality in nuclear medicine for human use, since it provides a higher sensitivity compared to SPECT [74]. PET requires the application of proton rich positron $\left(\beta^{+}\right)$emitters like ${ }^{11} \mathrm{C},{ }^{18} \mathrm{~F},{ }^{64} \mathrm{Cu},{ }^{68} \mathrm{Ga}$ or ${ }^{89} \mathrm{Zr}$ that decay by a subtype of beta decay, in which the proton inside the nucleus of a radionuclide is converted to a neutron while releasing a positron and a neutrino $[74,75]$. The positron travels 1 to $2 \mathrm{~mm}$ in matter until it is annihilated by an electron $\left(\beta^{+}\right)$, resulting in two $511 \mathrm{keV}$ photons emitted in opposing directions. PET scanners consist of an array of detectors that register the counts by coincidence on opposing detector elements (Figure 8). A detector element contains an array of scintillation crystals that convert the incident photon to an electric signal that is summed up and reconstructed by a three dimensional ordered subset expectation maximization (OSEM) algorithm [76, 77]. State of the art systems for clinical and preclinical use provide a volume resolution of $95 \mathrm{~mm}^{3}$ to $0.5 \mathrm{~mm}^{3}$, respectively, but cannot overcome the uncertainty of the traveling path of the positron $[78,79]$. 


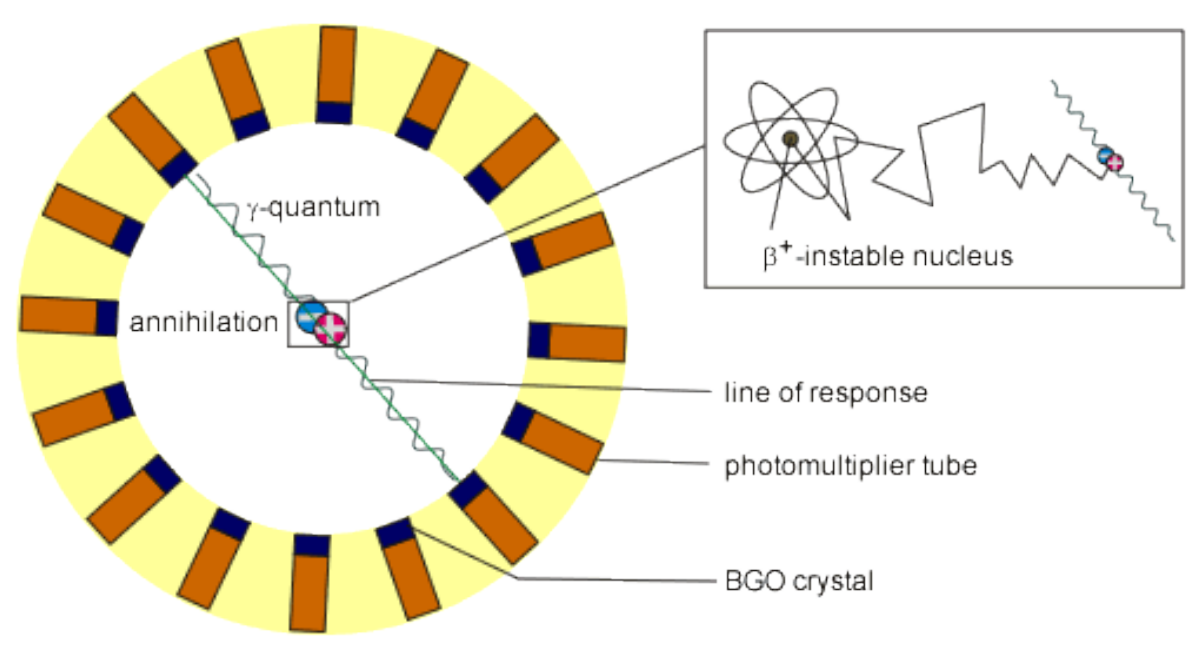

Figure 8: Principle of PET

PET scanners consist of an array of detectors that register $511 \mathrm{keV}$ photons ( $\gamma$-quantum) from annihilation of a positron $\left(\beta^{+}\right)$and an electron $\left(\beta^{-}\right)$by coincidence on opposing detector elements containing bismuth germanate (BGO) crystals. Image was adapted without modification from [80].

SPECT

In contrast to PET, SPECT detects photons of gamma emitting radionuclides that make SPECT very versatile in sense of applicable radionuclides, since gamma radiation, i.e. high energetic photons, is released to a certain amount with each decay. The higher the percentage of gamma emission of the decay, the more suitable is the use for SPECT. Typical SPECT tracers are ${ }^{99 \mathrm{~m}} \mathrm{Tc},{ }^{111} \mathrm{In},{ }^{123} \mathrm{I},{ }^{133} \mathrm{Xe}$ or ${ }^{201} \mathrm{TI}$. As already mentioned, ${ }^{99 \mathrm{~m}} \mathrm{Tc}$ is ideal for the application in SPECT, since it decays to ${ }^{99 \mathrm{~m}} \mathrm{Tc}$ by $100 \%$ (Figure 4)[64]. The SPECT system is based on the principle of scintigraphy, detecting emitted gamma radiation by gamma cameras [81]. The gamma camera or in particular the detector contains scintillation crystals similar to PET. In SPECT, one or more of the gamma cameras are rotated around the patient and detect emitted radiation from different directions in space. Spatial resolution is achieved by the use of a collimator [82]. These are thick sheets of lead with thousands of adjacent holes, ensuring only to detect photons that were emitted perpendicular to the detector (if a parallel hole collimator is used). Out of the acquisitions from different angles and the use of a collimator, the origin of the incident photon can be determined by reconstruction with a three dimensional OSEM algorithm. The major drawback of the collimators are the attenuation of more than $99 \%$ of incident photons, however impressively proving the 
sensitivity of this modality. The only opportunity to overcome this issue is to increase the applied activity to the patient. Up to $1110 \mathrm{MBq}$ of ${ }^{99 \mathrm{~m}} \mathrm{Tc}$-sestamibi are applied to patients undergoing a one day cardiac rest/stress examination acquiring an effective dose of $11 \mathrm{mSv}[83,84]$.

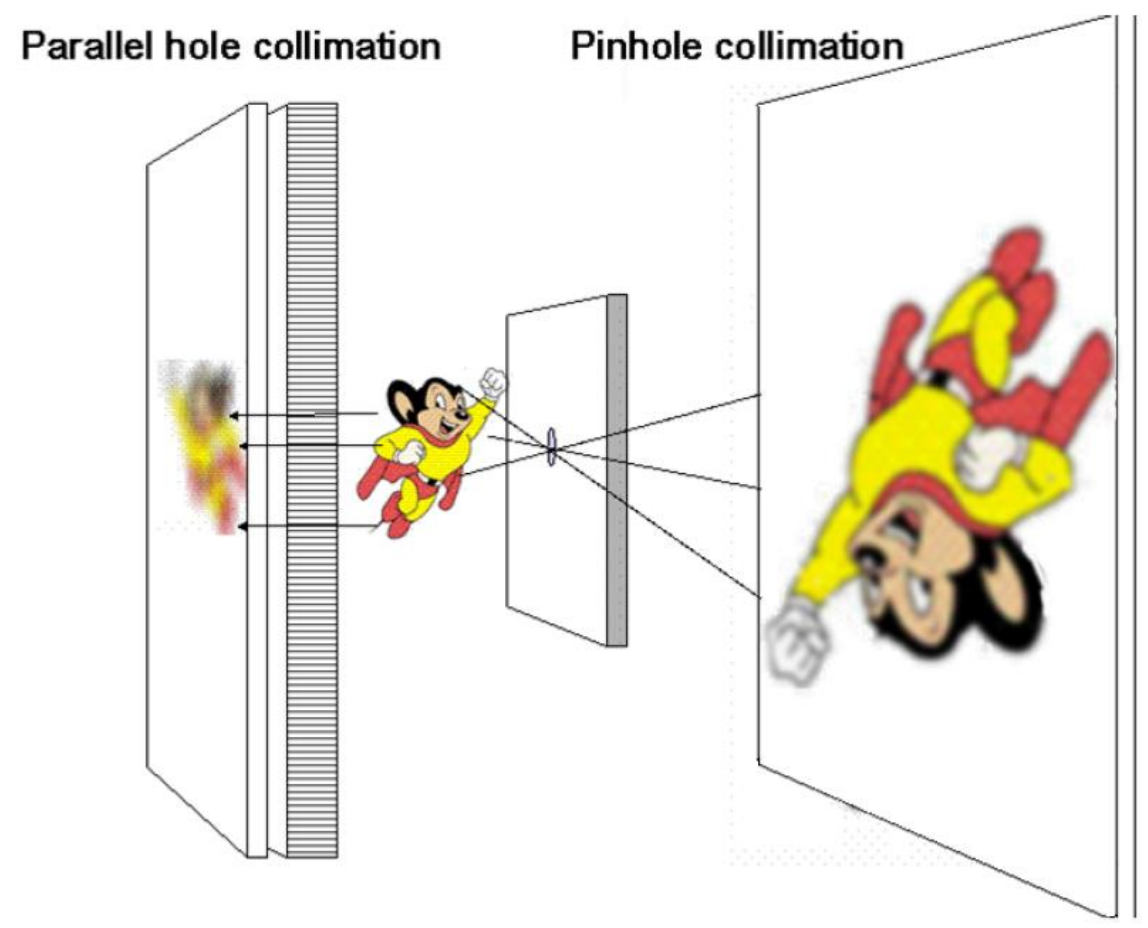

Figure 9: Parallel hole and pinhole collimators

Comparison of parallel hole and pinhole collimators. Image resolution can be degraded by the use of parallel hole collimators due to intrinsic camera blurring that is circumvented by the magnification resulting from the use of pinhole collimators. Image was adapted without modification from [82].

For the imaging of small animals like rodents, collimators based on the pinhole camera and intercept theorem were developed. The use of multi-pinhole collimators causes a magnification of the image on the detector and results in a higher resolution that is needed for preclinical imaging of small rodents $[82,85]$. The collimators have a multitude of pinholes that project a multitude of images on the detector decreasing the loss of sensitivity by the multitude of pinholes. A dedicated reconstruction algorithm based on the OSEM algorithm is used for reconstruction of the images. Latest developments in small animal SPECT imaging provide a spatial resolution of $0.25 \mathrm{~mm}[86]$. 


\subsection{The orthotopic tumor mouse model}

The development of novel imaging probes at preclinical stage for tumor visualization requires suitable animal models for a proper in vivo characterization in terms of tumor uptake and biodistribution. Since the imaging probes were intended for a later possible use in humans and the diagnosis of human diseases, xenograft mouse models have to be established, i.e. the implantation of human tumor cells or biopsies in immunodeficient mice [87]. The use of immunodeficient mice is mandatory to prevent repelling of human tumor cells by the host immune system. Several types of immunodeficient mice like athymic nude mice, severe combined immune deficient (SCID) mice or non-obese diabetic (NOD)-SCID mice can be used for the establishment of tumor xenograft models [87]. Athymic nude mice carry a deletion of the FOXN1gene, which is crucial for the development of the thymus and hair growth [88]. Since the maturation of thymocytes to T-lymphocytes (T-cells) takes place in the thymus, a lack thereof is connected with a lack of mature T-cells, however the number of B-cells remains unaffected [89]. Thus, athymic nude mice do not produce antibodies, have an impaired cell-mediated immune response, do not eliminate virulent or malignant cells and do not repel foreign tissue [89]. This makes athymic nude mice to an ideal host for human tumor cells. Since xenografted tumors for the development of solid tumors should ideally mimic the interaction of tumor cells with the stroma and extracellular matrix. The implantation of tumor cells in the organ of their origin, so-called orthotopic implantation, leads to a tumor growth and metastatic pattern comparable to the course of the disease in human organs [90-93]. 


\subsection{Aim of this study}

The aim of this work was the generation and characterization of novel nanobodies targeting the ion channel Kv10.1 as novel probes for in vivo tumor visualization in combination with multi-pinhole SPECT to enable the specific and non-invasive detection of tumor lesions early after probe administration. For this anti-Kv10.1 nanobodies had to be identified by phage display and characterized in vitro for specificity and binding properties. Since the SPECT system was not used for imaging of small animals before, the system and the imaging procedure including radiolabeling had to be validated using the clinically approved full $\operatorname{lgG}_{1}$ antibody Cetuximab targeting EGFR in tumor bearing mice. The suitability of tumor visualization with novel anti-EGFR nanobodies had to be assessed to generate an imaging approach with small-sized nanobodies for a successful in vivo application of anti-Kv10.1 nanobodies in future. 


\section{Material and Methods}

\subsection{Material}

Table 1: Equipment

\begin{tabular}{|c|c|}
\hline Instrument & Manufacturer \\
\hline Analytical balance & Sartorius, Göttingen, Germany \\
\hline Axiovert 200 & Zeiss, Jena, Germany \\
\hline Biacore 2000 & GE Healthcare (Biacore), Uppsala, Sweden \\
\hline ChemiDoc XRS system & Bio-Rad, München, Germany \\
\hline Cooling centrifuge 5403 and 5804 & Eppendorf, Hamburg, Germany \\
\hline Counting chamber for cells (Neubauer) & $\begin{array}{l}\text { Hecht Assistent, Sondheim v. d. Rhön, } \\
\text { Germany }\end{array}$ \\
\hline Dose calibrator & Biodex, Shirley, NY, US \\
\hline $\begin{array}{l}\text { Electrophoresis chamber system Xcell } \\
\text { SureLock Mini-Cell }\end{array}$ & $\begin{array}{l}\text { LifeTechnologies (Invitrogen), Darmstadt, } \\
\text { Germany }\end{array}$ \\
\hline Eppendorf Research pipettes & Eppendorf, Hamburg, Germany \\
\hline FACSaria & BD Bioscience, Heidelberg, Germany \\
\hline $\begin{array}{l}\text { Fladbed scanner with transparency unit } \\
\text { (Perfection V800) }\end{array}$ & Epson, Meerbusch, Germany \\
\hline In vivo small animal $\mathrm{CT}$ QuantumFX & Perkin Elmer, Waltham, MA, USA \\
\hline Incubator & Heraeus Instruments, Hanau, Germany \\
\hline Incubator for Bacteria innova 4340 & $\begin{array}{l}\text { Eppendorf (New Brunswick Scientific), } \\
\text { Hamburg, Germany }\end{array}$ \\
\hline Laboratory balance & Sartorius, Göttingen, Germany \\
\hline Magnetic stirrer IKAmag RET & IKA Werke, Staufen, Germany \\
\hline Mini Trans-Blot Cell & Bio-Rad, München, Germany \\
\hline Multi pinhole collimators HiSPECT & SciVis, Göttingen, Germany \\
\hline $\mathrm{pH}$ meter & Mettler-Toledo, Giessen, Germany \\
\hline PicoFuge & Agilent (Stratagene), Lake Forest, CA, US \\
\hline PipetBoy acu & Integra Bioscience, Biebertal, Germany \\
\hline Pipetman Classic Pipettes & Gilson, Villiers le Bel, France \\
\hline Powerpack P25 & Biometra, Göttingen, Germany \\
\hline SPECT gamma camera Prism XP3000 & $\begin{array}{l}\text { Philips (former Picker), Eindhoven, } \\
\text { Netherlands }\end{array}$ \\
\hline Spectrometer Ultrospec 1100 pro & $\begin{array}{l}\text { GE Healthcare (Amersham), Uppsala, } \\
\text { Sweden }\end{array}$ \\
\hline Tabletop centrifuge 5424 & Eppendorf, Hamburg, Germany \\
\hline Thermoblock TB1 & Biometra, Göttingen, Germany \\
\hline Thermomixer Compact 5350 & Eppendorf, Hamburg, Germany \\
\hline Tissue processor TP1020 & Leica, Wetzlar, Germany \\
\hline Vortex Genie 2 & Bender \& Hobein, Zurich, Switzerland \\
\hline Wallac Victor ${ }^{2}$ Multilabel counter 1420 & Perkin Elmer, Waltham, MA, USA \\
\hline
\end{tabular}




\begin{tabular}{ll}
\hline $\begin{array}{l}\text { Wallac Wizard 3" } 1480 \text { automatic gamma } \\
\text { counter }\end{array}$ & Perkin Elmer, Waltham, MA, USA \\
\hline Water Purification System arium 611 & Sartorius, Göttingen, Germany \\
\hline
\end{tabular}

Table 2: Chemicals

\begin{tabular}{|c|c|}
\hline Chemicals & Manufacturer \\
\hline ABTS-Solution & Roche, Mannheim, Germany \\
\hline Acetic acid & Merck, Darmstadt, Germany \\
\hline Agar & $\begin{array}{l}\text { LifeTechnologies (Gibco), Darmstadt, } \\
\text { Germany }\end{array}$ \\
\hline Agarose & $\begin{array}{l}\text { LifeTechnologies (Gibco), Darmstadt, } \\
\text { Germany }\end{array}$ \\
\hline Ammonium sulfate $\left(\left(\mathrm{NH}_{4}\right)_{2} \mathrm{SO}_{4}\right)$ & Sigma Aldrich, Schnelldorf, Germany \\
\hline Ampicillin & Roche, Mannheim, Germany \\
\hline Benzonase & $\begin{array}{l}\text { Merck Millipore (Novagen), Darmstadt, } \\
\text { Germany }\end{array}$ \\
\hline Bovine serum albumine (BSA) & Sigma Aldrich, Schnelldorf, Germany \\
\hline Calcium chloride $\left(\mathrm{CaCl}_{2}\right)$ & Sigma Aldrich, Schnelldorf, Germany \\
\hline Casein & Sigma Aldrich, Schnelldorf, Germany \\
\hline Coomassie Brilliant Blue G250 & Bio-Rad, München, Germany \\
\hline Cytoseal60 & Thermo Scientific, Darmstadt, Germany \\
\hline Dimethylformamide (DMF), anhydrous & SoluLink, San Diego, CA, US \\
\hline Dimethylsulfoxide (DMSO) & Sigma Aldrich, Schnelldorf, Germany \\
\hline Disodium phosphate $\left(\mathrm{Na}_{2} \mathrm{HPO}_{4}\right)$ & Sigma Aldrich, Schnelldorf, Germany \\
\hline Dithiothretiol (DTT) & Sigma Aldrich, Schnelldorf, Germany \\
\hline Ethanol abs. & Merck, Darmstadt, Germany \\
\hline Glucose & Merck, Darmstadt, Germany \\
\hline Glycerol & Merck, Darmstadt, Germany \\
\hline Glycine & Merck, Darmstadt, Germany \\
\hline Imidazole & Sigma Aldrich, Schnelldorf, Germany \\
\hline Isopropanol & Merck, Darmstadt, Germany \\
\hline $\begin{array}{l}\text { Isopropyl } \beta \text {-D-1-thiogalactopyranoside } \\
\text { (IPTG) }\end{array}$ & Roche, Mannheim, Germany \\
\hline Kanamycin & Roche, Mannheim, Germany \\
\hline Luria broth (LB) & $\begin{array}{l}\text { LifeTechnologies (Gibco), Darmstadt, } \\
\text { Germany }\end{array}$ \\
\hline Lysozyme & Roche, Mannheim, Germany \\
\hline Manganese chloride $\left(\mathrm{MnCl}_{2}\right)$ & Sigma Aldrich, Schnelldorf, Germany \\
\hline Methanol & Merck, Darmstadt, Germany \\
\hline MOPS & Sigma Aldrich, Schnelldorf, Germany \\
\hline Natrium chloride $(\mathrm{NaCl})$ & Sigma Aldrich, Schnelldorf, Germany \\
\hline Natriumdihydrogen phosphate $\left(\mathrm{NaH}_{2} \mathrm{PO}_{4}\right)$ & Merck, Darmstadt, Germany \\
\hline Nuclear fast red & Dako, Glostrup, Denmark \\
\hline Ortho-phosphoric acid & Merck, Darmstadt, Germany \\
\hline PEG8000 & Sigma Aldrich, Schnelldorf, Germany \\
\hline
\end{tabular}




\begin{tabular}{|c|c|}
\hline Peptone & $\begin{array}{l}\text { Life Technologies (Gibco), Darmstadt, } \\
\text { Germany }\end{array}$ \\
\hline Potassium acetate $\left(\mathrm{CH}_{3} \mathrm{CO}_{2} \mathrm{~K}\right)$ & Sigma Aldrich, Schnelldorf, Germany \\
\hline Potassium chloride (KCl) & Merck, Darmstadt, Germany \\
\hline Potassium phosphate $\left(\mathrm{KH}_{2} \mathrm{PO}_{4}\right)$ & Sigma Aldrich, Schnelldorf, Germany \\
\hline Rubidium chloride ( $\mathrm{RbCl}$ ) & Sigma Aldrich, Schnelldorf, Germany \\
\hline Silver nitrate $\left(\mathrm{AgNO}_{3}\right)$ & Merck, Darmstadt, Germany \\
\hline Skim milk powder & Bio-Rad, München, Germany \\
\hline Sodium acetate $\left(\mathrm{CH}_{3} \mathrm{COONa}\right)$ & Sigma Aldrich, Schnelldorf, Germany \\
\hline Sodium carbonate $\left(\mathrm{Na}_{2} \mathrm{CO}_{3}\right)$ & Sigma Aldrich, Schnelldorf, Germany \\
\hline Sodium dodecyl sulfate (SDS) & Sigma Aldrich, Schnelldorf, Germany \\
\hline Sodium thiosulfate $\left(\mathrm{Na}_{2} \mathrm{~S}_{2} \mathrm{O}_{3}\right)$ & Sigma Aldrich, Schnelldorf, Germany \\
\hline $\begin{array}{l}\text { Succinimidyl 6-hydraziniumnicotinate } \\
\text { hydrochloride (HyNic) }\end{array}$ & Solulink, San Diego, CA, US \\
\hline TALON Super Flow & GE Healthcare, Freiburg, Germany \\
\hline $\begin{array}{l}\text { Technetium-99m pertechnetate }\left({ }^{99 \mathrm{~m}} \mathrm{Tc}-\right. \\
\left.\mathrm{NaTcO}_{4}\right)\end{array}$ & UMG, Dept. Nulcear Medicine, Germany \\
\hline Tin chloride $\left(\mathrm{SnCl}_{2}\right)$ & Sigma Aldrich, Schnelldorf, Germany \\
\hline Tris-Base & Sigma Aldrich, Schnelldorf, Germany \\
\hline Tris-HCl & Merck, Darmstadt, Germany \\
\hline Trypan blue solution & Sigma Aldrich, Schnelldorf, Germany \\
\hline Tryptone & $\begin{array}{l}\text { LifeTechnologies (Gibco), Darmstadt, } \\
\text { Germany }\end{array}$ \\
\hline Tween 20 & Merck, Darmstadt, Germany \\
\hline Ultravist 370 & Bayer, Leverkusen, Germany \\
\hline Xylene & Sigma Aldrich, Schnelldorf, Germany \\
\hline Yeast extract & $\begin{array}{l}\text { LifeTechnologies (Gibco), Darmstadt, } \\
\text { Germany }\end{array}$ \\
\hline
\end{tabular}

Table 3: Antibodies

\begin{tabular}{|c|c|c|}
\hline Antibody & Application & Manufacturer \\
\hline $\begin{array}{l}\text { anti-Actin mouse mAb } \\
\text { \#MAB1501 }\end{array}$ & WB & $\begin{array}{l}\text { NEB (Cell Signaling), } \\
\text { Frankfurt a.M., Germany }\end{array}$ \\
\hline $\begin{array}{l}\text { anti-c-myc mouse mAb \#sc- } \\
40 \text {, clone } 9 \mathrm{E} 10\end{array}$ & FC, IP, WB & $\begin{array}{l}\text { Santa Cruz, Heidelberg, } \\
\text { Germany }\end{array}$ \\
\hline $\begin{array}{l}\text { anti-EGFR humanized anti- } \\
\text { human mAb Cetuximab }\end{array}$ & $\mathrm{FC}$, in vivo & Merck, Darmstadt, Germany \\
\hline anti-EGFR nanobody D10 & $\mathrm{FC}$, in vivo & in house \\
\hline anti-EGFR rabbit mAb \#2232 & WB & $\begin{array}{l}\text { NEB (Cell Signaling), } \\
\text { Frankfurt a.M., Germany }\end{array}$ \\
\hline $\begin{array}{l}\text { anti-EGFR rabbit mAb \#MA5- } \\
16359 \text {, clone SP9 }\end{array}$ & IHC & $\begin{array}{l}\text { Thermo Scientific, } \\
\text { Darmstadt, Germany }\end{array}$ \\
\hline anti-His mouse $\mathrm{mAb} \#$ & ELISA, FC, WB & $\begin{array}{l}\text { Merck Millipore (Novagen), } \\
\text { Darmstadt, Germany }\end{array}$ \\
\hline anti-Kv10.1 mouse mAb 33 & IP & in house \\
\hline anti-Kv10.1 nanobody C4 & IP, IHC, SPR, ELISA & in house \\
\hline
\end{tabular}




\begin{tabular}{|c|c|c|}
\hline anti-Kv10.1 nanobody D9 & IP, IHC, SPR, ELISA & in house \\
\hline anti-Kv10.1 rabbit pAb 9391 & WB & in house \\
\hline $\begin{array}{l}\text { goat anti-mouse mAb - HRP } \\
\text { \#NA931VS }\end{array}$ & ELISA, WB & $\begin{array}{l}\text { GE Healthcare (Amersham), } \\
\text { Freiburg, Germany }\end{array}$ \\
\hline goat anti-mouse mAb - PE & FC & $\begin{array}{l}\text { Santa Cruz, Heidelberg, } \\
\text { Germany }\end{array}$ \\
\hline $\begin{array}{l}\text { goat anti-rabbit mAb - HRP } \\
\text { \#NA934VS }\end{array}$ & ELISA, WB & $\begin{array}{l}\text { GE Healthcare (Amersham), } \\
\text { Freiburg, Germany }\end{array}$ \\
\hline $\begin{array}{l}\text { Histofine Simple Stain Max } \\
\text { PO-R (anti-rabbit-HRP) }\end{array}$ & IHC & $\begin{array}{l}\text { Nichirei Biosciences, Tsukiji, } \\
\text { Japan }\end{array}$ \\
\hline $\begin{array}{l}\text { human IgG1 isotype \#009- } \\
000-003\end{array}$ & $\mathrm{FC}$, in vivo & $\begin{array}{l}\text { Dianova (Jackson } \\
\text { Immunolabs), Hamburg, } \\
\text { Germany }\end{array}$ \\
\hline $\begin{array}{l}\text { irrelevant control nanobody } \\
\text { F5 }\end{array}$ & $\mathrm{FC}$, in vivo & in house \\
\hline $\begin{array}{l}\text { Protein G - AlexaFluor } 488 \\
\text { \#P11065 }\end{array}$ & FC & $\begin{array}{l}\text { LifeTechnologies, Darmstadt, } \\
\text { Germany }\end{array}$ \\
\hline
\end{tabular}

Table 4: Cell lines

\begin{tabular}{llll}
\hline Cell line & Description & DSMZ No & Medium \\
\hline A431 & human epidermoid carcinoma & ACC 91 & 90\% DMEM, 10\% FCS \\
HEK293 & human embryonic kidney & ACC 635 & $90 \%$ DMEM, 10\% FCS \\
MDA-MB-231 & human breast carcinoma & ACC 732 & $90 \%$ DMEM, 10\% FCS \\
MDA-MB-468 & human breast carcinoma & ACC 738 & $90 \%$ DMEM, 10\% FCS \\
\hline $\begin{array}{l}\text { Transfected cell } \\
\text { line }\end{array}$ & Containing plasmid & & \\
HEK293 & pcDNA3-Kv10.1-venus & & $90 \%$ DMEM, 10\% FCS \\
HEK293 & pcDNA3- $\varnothing$-venus (empty plasmid) & & $90 \%$ DMEM, 10\% FCS \\
HEK293 & pTracer-Kv10.1 & & $90 \%$ DMEM, 10\% FCS \\
HEK293 & pTracer- $\varnothing$ (empty plasmid) & & $90 \%$ DMEM, 10\% FCS \\
\hline
\end{tabular}

Table 5: Cell culture components

\begin{tabular}{ll}
\hline Cell culture components & Manufacturer \\
\hline DMEM high glucose (4.5 g/l) with GlutaMAX & $\begin{array}{l}\text { Life Technologies (Invitrogen), Darmstadt, } \\
\text { Germany }\end{array}$ \\
Fetal calf serum (FCS) & $\begin{array}{l}\text { GE Healthcare (PAA), Cölbe, Germany } \\
\text { Merck Millipore (Biochrom), Darmstadt, } \\
\text { EDTA (5 mM) }\end{array}$ \\
Germany \\
OptiMEM & $\begin{array}{l}\text { Life Technologies (Invitrogen), Darmstadt, } \\
\text { Germany }\end{array}$ \\
PBS (1x) without Ca ${ }^{2+}$ and $\mathrm{Mg}^{2+}$ & $\begin{array}{l}\text { Life Technologies (Invitrogen), Darmstadt, } \\
\text { Germany }\end{array}$ \\
Trypsin 0.05\% / EDTA (0.02\%) & $\begin{array}{l}\text { Merck Millipore (Biochrom), Darmstadt, } \\
\text { Germany }\end{array}$ \\
\hline
\end{tabular}


Table 6: E. coli strains and phages

\begin{tabular}{|c|c|}
\hline E. coli strain & Genotype \\
\hline BL21DE & E. coli $\mathrm{B}, \mathrm{F}-, d c m$, omp $\mathrm{T}$, $h s d \mathrm{~S}(\mathrm{rB}-\mathrm{mB}-)$, gald \\
\hline $\mathrm{TG} 1_{\mathrm{TR}}$ & K-12 supE thi-1 $\Delta$ (lac-proAB) $\Delta$ (mcrB-hsdSM)5, $\left(r_{K}^{-} m_{K}{ }^{-}\right)$ \\
\hline \multicolumn{2}{|l|}{ Phages } \\
\hline KM13 Helper Phage & NEB, Frankfurt a.M, Germany \\
\hline M13KO7 Helper Phage & NEB, Frankfurt a.M, Germany \\
\hline
\end{tabular}

Table 7: Bacterial growth media

\begin{tabular}{ll}
\hline Growth media & Composition \\
\hline 2 YT & $1.6 \%(\mathrm{w} / \mathrm{v})$ tryptone, $1 \%(\mathrm{w} / \mathrm{v})$ yeast extract, $0.5 \%(\mathrm{w} / \mathrm{v})$ \\
& $\mathrm{NaCl}, \mathrm{pH} 7.0$ \\
$2 \mathrm{YT}$-agar & $1.5 \%(\mathrm{w} / \mathrm{v})$ agar in $2 \mathrm{YT}$ medium \\
LB & $1 \%(\mathrm{w} / \mathrm{v})$ peptone, $0.5 \%(\mathrm{w} / \mathrm{v})$ yeast extract, $1 \%(\mathrm{w} / \mathrm{v}) \mathrm{NaCl}$, \\
& $\mathrm{pH} \mathrm{7.0}$ \\
LB-agar & $1.5 \%(\mathrm{w} / \mathrm{v})$ agar in LB medium \\
\hline
\end{tabular}

Table 8: Commercial buffers

\begin{tabular}{|c|c|c|}
\hline Buffer & Catalog number & Manufacturer \\
\hline Antibody diluent solution & S202230-2 & Dako, Glostrup, Denmark \\
\hline Antigen-retrieval buffer & S170084-2 & Dako, Glostrup, Denmark \\
\hline HBS-EP & BR100188 & $\begin{array}{l}\text { GE Healthcare (Biacore), Uppsala, } \\
\text { Sweden }\end{array}$ \\
\hline $\begin{array}{l}\text { ImmPACT DAB Peroxidase } \\
\text { substrate }\end{array}$ & SK4105 & Vector Laboratories, Peterborough, UK \\
\hline Peroxidase blocking solution & S202386-2 & Dako, Glostrup, Denmark \\
\hline RIPA Buffer & R0278 & Sigma Aldrich, Schnelldorf, Germany \\
\hline Seablock & 37527 & $\begin{array}{l}\text { Thermo Scientific, Darmstadt, } \\
\text { Germany }\end{array}$ \\
\hline
\end{tabular}


Table 9: Commercial kits

\begin{tabular}{lll}
\hline Kit & Application & Manufacturer \\
\hline BCA Protein Assay Kit & $\begin{array}{l}\text { Determination of protein } \\
\text { concentration }\end{array}$ & $\begin{array}{l}\text { Thermo Scientific, } \\
\text { Darmstadt, Germany }\end{array}$ \\
$\begin{array}{l}\text { Bug Buster Protein } \\
\text { Extraction Reagent }\end{array}$ & $\begin{array}{l}\text { Protein Extraction from } \\
\text { bacteria }\end{array}$ & $\begin{array}{l}\text { Merck Millipore (Novagen), } \\
\text { San Diego, CA, US }\end{array}$ \\
CRS Kit for tricarbonyl & Tricarbonyl labeling & $\begin{array}{l}\text { Paul Scherrer Institut, } \\
\text { Villingen, Switzerland } \\
\text { ECL Kit }\end{array}$ \\
NucleoSpin Plasmid Kit & Developing of WB & $\begin{array}{l}\text { Merck Millipore, Darmstadt, } \\
\text { Germany } \\
\text { Macherey Nagel, Düren, } \\
\text { Germany }\end{array}$ \\
\hline
\end{tabular}

Table 10: Buffer compositions

\begin{tabular}{|c|c|}
\hline Buffer & Composition \\
\hline Colloidal Coomassie Staining (stock) & $\begin{array}{l}0.1 \%(\mathrm{w} / \mathrm{v}) \text { Coomassie Brilliant Blue G250, } \\
2 \%(\mathrm{w} / \mathrm{v}) \text { ortho-phosphoric acid, } 10 \%(\mathrm{w} / \mathrm{v}) \\
\left(\mathrm{NH}_{4}\right)_{2} \mathrm{SO}_{4}\end{array}$ \\
\hline $\begin{array}{l}\text { Colloidal Coomassie Staining (working } \\
\text { solution) }\end{array}$ & $\begin{array}{l}80 \%(v / v) \text { Coomassie stock solution, } 20 \% \\
(v / v) \text { methanol }\end{array}$ \\
\hline Conjugation Buffer & $100 \mathrm{mM} \mathrm{Na}_{2} \mathrm{HPO}_{4}, 150 \mathrm{mM} \mathrm{NaCl}, \mathrm{pH} 6.0$ \\
\hline Detection buffer solution & $\begin{array}{l}100 \mathrm{mM} \text { Tris-Base, } 100 \mathrm{mM} \mathrm{NaCl}, 5 \mathrm{mM} \\
\mathrm{MgCl}_{2}, \mathrm{pH} 8.8\end{array}$ \\
\hline EP & $\begin{array}{l}50 \mathrm{mM} \mathrm{CH}_{3} \mathrm{COONa}, 0.1 \mathrm{M} \mathrm{NaCl}, 250 \mathrm{mM} \\
\text { Imidazole } \mathrm{pH} 7.8\end{array}$ \\
\hline Modification Buffer & $100 \mathrm{mM} \mathrm{Na}_{2} \mathrm{HPO}_{4}, 150 \mathrm{mM} \mathrm{NaCl}, \mathrm{pH} 7.4$ \\
\hline Phosphate-buffered saline (PBS) (10x) & $\begin{array}{l}\text { 1.4 M NaCl, } 25 \mathrm{mM} \mathrm{KCl}^{2} 20 \mathrm{mM} \mathrm{KH}_{2} \mathrm{PO}_{4} \\
100 \mathrm{mM} \mathrm{Na}_{2} \mathrm{HPO}_{4}, \mathrm{pH} 7.4\end{array}$ \\
\hline RF1 & $\begin{array}{l}100 \mathrm{mM} \mathrm{RbCl}, 50 \mathrm{mM} \mathrm{MnCl}_{2}, 30 \mathrm{mM} \\
\text { potassium acetate, } 10 \mathrm{mM} \mathrm{CaCl}_{2}, 15 \%(\mathrm{w} / \mathrm{v}) \\
\text { glycerin, pH } 5.8\end{array}$ \\
\hline RF2 & $\begin{array}{l}100 \mathrm{mM} \text { MOPS, } 10 \mathrm{mM} \mathrm{RbCl}, 75 \mathrm{mM} \mathrm{CaCl}_{2}, \\
15 \% \text { (w/v) glycerin, } \mathrm{pH} 6.8\end{array}$ \\
\hline Tris-buffered saline (TBS) (10x) & $1.4 \mathrm{M} \mathrm{NaCl}, 500$ mM Tris-HCl, pH 7.5 \\
\hline WP1 & $50 \mathrm{mM} \mathrm{CH}_{3} \mathrm{COONa}, 1 \mathrm{M} \mathrm{NaCl}$, pH 7.8 \\
\hline WP2 & $50 \mathrm{mM} \mathrm{CH} 3 \mathrm{COONa}, 0.1 \mathrm{M} \mathrm{NaCl}, \mathrm{pH} 7.8$ \\
\hline
\end{tabular}

Table 11: Consumables

\begin{tabular}{ll}
\hline Consumables & Manufacturer \\
\hline 96 well plates & Sarstedt, Nümbrecht, Germany \\
96 well plates, deep wells & Sarstedt, Nümbrecht, Germany \\
96 well plates, round bottom & Sarstedt, Nümbrecht, Germany \\
Amicon Ultracel Spin Filters 3 K and 10 K & Merck Millipore, Cork, Ireland \\
MWCO & \\
CM5 Chip for Biacore 2000 & GE Healthcare (Biacore), Uppsala, Sweden \\
\hline
\end{tabular}




\begin{tabular}{|c|c|}
\hline Counting tubes for biodistribution analysis & VWR, Hannover, Germany \\
\hline Cuvettes for photometry & Sarstedt, Nümbrecht, Germany \\
\hline Dynabeads M-450 Epoxy & Life Technologies, Darmstadt, Germany \\
\hline Eppendorf LoBind tubes & Eppendorf, Hamburg, Germany \\
\hline Eppendorf SafeLock tubes & Eppendorf, Hamburg, Germany \\
\hline $\begin{array}{l}\text { FACS tubes Falcon } 5 \mathrm{ml} \text { polystyrene round } \\
\text { bottom }\end{array}$ & Corning, New York, NY, US \\
\hline Falcon centrifugation tubes 15 and $50 \mathrm{ml}$ & Corning, New York, NY, US \\
\hline Filtered pipette tips (Art Tips) & Thermo Scientific, Darmstadt, Germany \\
\hline Hollow needles ( $25 \mathrm{G}-12 \mathrm{G}$ ) & BD Bioscience, Heidelberg, Germany \\
\hline Hybond Nitrocellulose Membrane & $\begin{array}{l}\text { GE Healthcare (Amersham), Freiburg, } \\
\text { Germany }\end{array}$ \\
\hline Insulin syringe (30 G) & B.Braun, Melsungen, Germany \\
\hline $\begin{array}{l}\text { ITLC SG Strips Glas microfiber } \\
\text { chromatography paper impregnated with } \\
\text { silica gel }\end{array}$ & Agilent, Lake Forest, CA, US \\
\hline Maxisorp plates (96 well) & $\begin{array}{l}\text { Thermo Scientific (Nunc), Darmstadt, } \\
\text { Germany }\end{array}$ \\
\hline $\begin{array}{l}\text { Novex Bis-Tris (4 - 12\%) and Tris-Acetate (3- } \\
8 \%) \text { gels }\end{array}$ & $\begin{array}{l}\text { Life Technologies (Invitrogen), Darmstadt, } \\
\text { Germany }\end{array}$ \\
\hline Pipette tips & Sarstedt, Nümbrecht, Germany \\
\hline Protein G magnetic beads & NEB, Frankfurt a.M., Germany \\
\hline Serological pipettes & Sarstedt, Nümbrecht, Germany \\
\hline Surgical sutures (4/0, absorbable) & B.Braun, Melsungen, Germany \\
\hline $\begin{array}{l}\text { Syringe filters } 0.2 \mu \mathrm{m} \text {, Polyethersulfone (PES) } \\
\text { membrane }\end{array}$ & Sartorius, Göttingen, Germany \\
\hline Syringes $(0.5-10 \mathrm{ml})$ & BD Bioscience, Heidelberg, Germany \\
\hline Tissue embedding cassettes (Rotilabo) & Roth, Karlsruhe, Germany \\
\hline Vaccum filter units & Thermo Scientific, Darmstadt, Germany \\
\hline Whatman filter paper & Bio-Rad, München, Germany \\
\hline
\end{tabular}

Table 12: Anesthetics

\begin{tabular}{ll}
\hline Anesthetic & Manufacturer \\
\hline Isoflurane & Abbvie, Ludwigshafen, Gemany \\
Ketamine & Medistar, Ascheberg, Germany \\
Rompun (Xylariem) & Ecuphar, Greifswald, Germany \\
\hline
\end{tabular}

Table 13: Software

\begin{tabular}{ll}
\hline Software & Manufacturer \\
\hline BIAevaluation v. 4.1.1 & GE Healthcare (Biacore), Uppsala, Sweden \\
\hline Excel 2007 & Microsoft, Redmond, WA, USA \\
\hline FlowJo v. 7.6.5 for Windows & TreeStar Inc., Ashland, OR, USA \\
GraphPad Prism v. 6.01 for Windows & GraphPad Software, La Jolla, CA, USA \\
Scry v.5.0 & Kuchl \& Sautter GbR, Bad Teinach, Germany \\
\hline
\end{tabular}




\subsubsection{Vector constructs}

For transfection in eukaryotic cells pTracer-Kv10.1 and pTracer- $\varnothing$ (empty plasmid) as well as pcDNA3-Kv10.1-venus and pcDNA3- $\varnothing$-venus (empty plasmid; Life Technologies, Darmstadt, Germany) were used. Nanobodies were presented on a pHEN vector.

\subsubsection{Animals}

Female and male athymic nude mice (NMRI-Foxn $1^{\text {nu/nu}}$ ) were initially obtained from Charles River (Sulzfeld, Germany) and bred in the Central Animal Facility, University Medical Center Göttingen.

\subsubsection{Antigen $\mathrm{H} 1 \mathrm{X}$}

The antigen $\mathrm{H} 1 \mathrm{X}$ is a $24 \mathrm{kDa}$ human $\mathrm{Kv} 10.1$ fusion protein consisting of thioredoxin (TRX), a 6xHis tag, a part of the Threonine-Serine-Glutamate-Lysine-Poredomain (AA 374 - 452), a linker and a part of the C-terminal assembly domain (CAD, AA 872 - 932).

Amino acid sequence of H1X:

MSDKIIHLTDDSFDTDVLKADGAILVDFWAEWCGPCKMIAPILDEIADEYQGKLTVAKLNIDQNPG TAPKYGIRGIPTLLLFKNGEVAATKVGALSKGQLKEFLDANLAGSGSGHMHHHHHHSSGLVPRGS GMKETAAAKFERQHMDSPDLGTDDDDKAMGDYEIFDEDTKTIPTTAGCPTSDGHWPPYQFNGS GSRKWEGGPSKNSVYISSLDFTMTSLTSV

\subsubsection{Standards}

For determination of molecular weights the broad range color prestained protein standard (\#7711 and 7712, NEB, Frankfurt a.M., Germany) was used. 


\subsection{Methods}

\subsubsection{Phage display}

The term "phage display" describes an iterative process to select and enrich single antibodies out of trillions $\left(10^{13}\right)$ of clones with help of bacteriophages. Phages can infect bacteria, replicate and express the protein coded on dedicated vectors of the host. The advantage of phages is that they express the intact protein on their surface and enable binding to protein of interest. One round of phage display consisted of the production of phages, selection on the target protein, infection of bacteria with the selected phages and production of the selected nanobodies with subsequent screening or another round of phage display starting with infection of the bacteria with phages.

Phages are extremely volatile, thus the work with phages required special safety precautions such as special laboratories with dedicated fume hoods for phage work, use of filtered tips and decontamination of all contaminated material in bleach for at least $24 \mathrm{~h}$.

\section{Preparation of phages}

$50 \mathrm{ml}$ 2YT medium supplemented with ampicillin $(100 \mu \mathrm{g} / \mathrm{ml})$ were inoculated with $25 \mu \mathrm{l}$ of the antibody library or the output culture after each round of selection. The optical density at $600 \mathrm{~nm}\left(\mathrm{OD}_{600}\right)$ of the culture was 0.1 at the time of inoculation. The culture was incubated $\left(37^{\circ} \mathrm{C}, 250 \mathrm{rpm}\right)$ until $\mathrm{OD}_{600}>0.4$ and $<0.6$. The $\mathrm{OD}_{600}$ must not exceed 0.6 because the culture had to remain in the exponential growth phase. The bacteria form pili during the exponential growth phase, which were needed by the phages for infection the bacteria. An $\mathrm{OD}_{600}$ of 1 represents $5 \times 10^{8}$ bacteria per $\mathrm{ml}$.

The culture was infected with an 20x excess of KM13 helper phage and incubated without shaking ( $30 \mathrm{~min}, 37^{\circ} \mathrm{C}$ ). The culture was spun down (15 min, $4000 \mathrm{rpm}, \mathrm{RT}$ ) and the pellet was resuspended in $250 \mathrm{ml}$ 2YT medium supplemented with ampicillin $(100 \mu \mathrm{g} / \mathrm{ml})$ and kanamycin $(50 \mu \mathrm{g} / \mathrm{ml})$ and incubated overnight $\left(30^{\circ} \mathrm{C}, 250 \mathrm{rpm}\right)$. The following day, the culture was split and transferred in five $50 \mathrm{ml}$ centrifugation tubes and spun down (20 min, $4000 \mathrm{rpm}, 4^{\circ} \mathrm{C}$ ). The supernatants were split in $25 \mathrm{ml}$ fractions and transferred in clean $50 \mathrm{ml}$ centrifugation tubes. Phages were precipitated by 
addition of $5 \mathrm{ml}$ ( $1 / 5$ of the volume) 20\% PEG8000, $\mathrm{NaCl} 2.5 \mathrm{mM}$ to each fraction and subsequent incubation on ice $(1 \mathrm{~h})$. The precipitated phages were spun down (15 min, $4000 \mathrm{rpm}, 4^{\circ} \mathrm{C}$ ), the supernatant was discarded and two pellets were pooled in $1 \mathrm{ml}$ PBS, transferred in $1.5 \mathrm{ml}$ Eppendorf tubes and spun (2 min, $14000 \mathrm{rpm}, 4^{\circ} \mathrm{C}$ ) to remove remaining bacteria. The supernatant was transferred in clean $1.5 \mathrm{ml}$ Eppendorf tubes and the phages were precipitated again by addition of $200 \mu \mathrm{l}(1 / 5$ of the volume) $20 \%$ PEG8000, $\mathrm{NaCl} 2.5 \mathrm{mM}$ to each tube. Following incubation on ice (30 min) and centrifugation $\left(5 \mathrm{~min}, 14000 \mathrm{rpm}, 4^{\circ} \mathrm{C}\right)$, the pellet was resuspended in $1 \mathrm{ml}$ PBS supplemented with $15 \%$ glycerol and stored at $-80^{\circ} \mathrm{C}$.

\section{Selection on immobilized protein H1X with previous depletion on TRX}

The process of selection is the actual step of phage display. The phages express the nanobody on the so-called head of the phage, which allows the binding, i.e. a selection on a protein of interest. A previous depletion step can lower the number of phages used for the actual selection and reduces unspecific binding, i.e. not on the target, thus resulting in more positive hits in subsequent screening.

The selection process should ideally be started in the morning with inoculation of a $20 \mathrm{ml}$ culture $E$. coli $\mathrm{TG} 1_{\mathrm{TR}}$ in $2 \mathrm{YT}$ minimal medium and with incubation for approximately 6 to $8 \mathrm{~h}\left(37^{\circ} \mathrm{C}, 250 \mathrm{rpm}\right)$ until an $\mathrm{OD}_{600}$ between 0.4 and 0.6 .

One $1 \mathrm{ml}$ aliquot of the frozen phages was thawed on ice and supplemented with $200 \mu \mathrm{l} 20 \%$ PEG8000, $2.5 \mathrm{mM} \mathrm{NaCl}$ to precipitate the phages (30 min, ice) and remove the cryo-preservative glycerol. The phages were spun $\left(5 \mathrm{~min}, 14000 \mathrm{rpm}, 4^{\circ} \mathrm{C}\right)$ and the pellet was resuspended in $1 \mathrm{ml}$ bovine serum albumin (BSA) (2\% in PBS) and incubated in an overhead rotator $\left(1 \mathrm{~h}, 4^{\circ} \mathrm{C}\right)$ for blocking. The immobilized TRX and H1X on magnetic beads (M450 Epoxy beads) were blocked in BSA (2\%) as well. For depletion on TRX the blocking solution was removed from the beads and the resuspended phages were incubated with the immobilized proteins $\left(2 \mathrm{~h}\right.$, overhead, $\left.4^{\circ} \mathrm{C}\right)$. A $5 \mu \mathrm{l}$ aliquot was recovered before incubation on TRX for later determination of the selection efficiency and referred as "input". After incubation, the depleted phages, i.e. the supernatant of the TRX slurry, were incubated $\left(2 \mathrm{~h}\right.$, overhead, $\left.4^{\circ} \mathrm{C}\right)$ on immobilized $\mathrm{H} 1 \mathrm{X}$ for selection. For elution of the bound phages, the beads were washed $9 \mathrm{x}$ with $1 \mathrm{ml}$ PBS Tween (0.1\%) and twice with $1 \mathrm{ml}$ PBS. Phages were eluted by incubation 
with $500 \mu$ trypsin $(1 \mathrm{mg} / \mathrm{ml}$ ) (30 min, overhead, RT), $500 \mu \mathrm{l}$ PBS were added and the supernatant was kept and referred as "output". An aliquot of $5 \mu \mathrm{l}$ was stored for subsequent determination of the selection efficiency. The remaining $995 \mu$ l output were diluted with $4 \mathrm{ml} 2 \mathrm{YT}$ medium and used for infection of $5 \mathrm{ml}$ of the $E$. coli TG1 $1_{\mathrm{TR}}$ culture $\left(0.4<\mathrm{OD}_{600}<0.6\right)$ inoculated in the morning with subsequent incubation without agitation $\left(30 \mathrm{~min}, 37^{\circ} \mathrm{C}\right)$. The infected bacteria were spun (10 min, $4000 \mathrm{rpm}$, RT) and the pellet was resuspended in $3 \mathrm{ml} 2 \mathrm{YT}$ medium. $500 \mu \mathrm{l}$ of the infected bacterial culture were plated out on square plates with 2YT-Agar supplemented with ampicillin $(100 \mu \mathrm{g} / \mathrm{ml})$ and glucose $(2 \%)$ and incubated overnight at $30^{\circ} \mathrm{C}$. The bacteria were harvested with $2 \times 3 \mathrm{ml} 2 \mathrm{YT}$ medium containing ampicillin (100 $\mu \mathrm{g})$ and glucose (2\%). The bacterial suspension was spun (10 min, $4000 \mathrm{rpm}, 4^{\circ} \mathrm{C}$ ) and the pellet was resuspended in the same volume $2 \mathrm{YT}$ medium containing ampicillin and glucose as the pellet resulted an $\mathrm{OD}_{600}$ of approx. 100. Glycerol was added to a final concentration of $15 \%(\mathrm{v} / \mathrm{v})$ and aliquots of $1 \mathrm{ml}$ were stored at $-80^{\circ} \mathrm{C}$ and used for the next round of phage display.

\section{Determination of selection efficiency}

For determination of the selection efficiency, the $5 \mu$ input and output aliquots were diluted (1:100) in 2YT medium and titrated in $10 \mathrm{x}$ dilution steps until $10^{-10}$ and $10^{-7}$ for input and output, respectively. The diluted phages were used for infection of the same volume of the previously inoculated $E$. coli TG1 $1_{\mathrm{TR}}$ culture $\left(0.4<\mathrm{OD}_{600}<0.6\right)$. The bacteria were incubated without agitation $\left(30 \mathrm{~min}, 37^{\circ} \mathrm{C}\right.$ ) for infection. $100 \mu \mathrm{l}$ of the infected bacteria were plated out on 2YT-Agar supplemented with ampicillin $(100 \mu \mathrm{g} / \mathrm{ml})$ and glucose $(2 \%)$ and incubated overnight at $37^{\circ} \mathrm{C}$. The following day, the colonies were counted to determine the selection efficiency.

\section{Conservation of the selected clones}

Two 96 deep well plates were prepared with $450 \mu \mathrm{l}$ 2YT medium containing ampicillin $(100 \mu \mathrm{g} / \mathrm{ml})$ and glucose $(2 \%)$ per well. 186 colonies of the output cultures (3 sterile controls) were picked and incubated overnight $\left(37^{\circ} \mathrm{C}, 250 \mathrm{rpm}\right)$. The next day, $150 \mu \mathrm{l}$ of the bacteria cultures were transferred to round bottom 96 well plates and $30 \mu \mathrm{l}$ glycerol (80\%) were administered per well as cryo-conservative before storing the plates at $-80^{\circ} \mathrm{C}$. 


\subsubsection{Screening}

For screening of the selected clones, the nanobodies had to be expressed first. Therefore, two 96 deep well plates were prepared with $435 \mu \mathrm{l} 2$ YT medium containing ampicillin $(100 \mu \mathrm{g} / \mathrm{ml})$ and glucose $(2 \%)$ per well and were incubated with $15 \mu \mathrm{l}$ of the fresh or cryoculture. The plates were incubated 1.5 (fresh culture) or $2.5 \mathrm{~h}$ (cryoculture) $\left(37^{\circ} \mathrm{C}, 250 \mathrm{rpm}\right) . \quad$ At $\mathrm{OD}_{600}=0.5,50 \mu \mathrm{l}$ Isopropyl $\beta-\mathrm{D}-1-$ thiogalactopyranoside (IPTG) (10x, diluted in 2YT medium) were added to each well to induce the nanobody expression. The plates were incubated overnight $\left(30^{\circ} \mathrm{C}, 250 \mathrm{rpm}\right)$ and spun $\left(10 \mathrm{~min}, 4000 \mathrm{rpm}, 4^{\circ} \mathrm{C}\right.$ ) the next morning. The supernatants were used for screening by the enzyme linked immunosorbent assay (ELISA).

\subsubsection{ELISA}

The ELISA is a colorimetric assay that was used to reveal nanobodies bound to their target protein. The assay was performed on Maxisorp Plates, so the proteins of interest, such as $\mathrm{H} 1 \mathrm{X}$ and $\mathrm{TRX}$ as negative control $(10 \mu \mathrm{g} / \mathrm{ml}, 50 \mu \mathrm{l} /$ well $)$, were immobilized by absorption on the plastic (overnight, $250 \mathrm{rpm}$, room temperature (RT)). For blocking, each well was incubated with $200 \mu \mathrm{l}$ BSA (2\%) (1 h, $250 \mathrm{rpm}, \mathrm{RT})$. For screening, $100 \mu \mathrm{l}$ of the supernatant were incubated $(1 \mathrm{~h}, 250 \mathrm{rpm}, \mathrm{RT})$ and subsequently washed with 3x $200 \mu$ PBS with Tween (0.1\%) (PBST) and 3x $200 \mu$ PBS. Binding of the nanobodies was revealed by consecutive incubation with an anti-His and an anti-mouse mAb coupled with a horseradish peroxidase (HRP) (each $5 \mu \mathrm{g} / \mathrm{ml}$ in BSA (2\%); $1 \mathrm{~h}, 250 \mathrm{rpm}, \mathrm{RT}$ ) with three PBST and PBS washing steps, each performed between the different incubation steps. The binding was visualized by addition of $100 \mu \mathrm{l}$ 2,2'-azino-bis(3-ethylbenzothiazoline-6-sulphonic acid) (ABTS) per well resulting in a blue staining that was measured at $405 \mathrm{~nm}$ in a multiwell counter (Wallac Victor ${ }^{2}$ Multilabel counter 1420).

\subsubsection{Production of chemically competent E. coli BL21(DE) cells}

Competent E. coli cells were produced by modification of the cell wall, which mediates DNA uptake. A $3 \mathrm{ml}$ starter culture (Luria broth (LB) medium) was inoculated with a 
single bacterial colony from a LB plate grown with colonies of chemically competent $E$. coli $\mathrm{BL} 21(\mathrm{DE} 3)$ cells and incubated overnight $\left(37^{\circ} \mathrm{C}, 250 \mathrm{rpm}\right) .100 \mathrm{ml} \mathrm{LB}$ medium were inoculated with the starter culture $\left(\mathrm{OD}_{600}=0.1\right)$ and incubated until $\mathrm{OD}_{600}=0.6\left(37^{\circ} \mathrm{C}\right.$, $250 \mathrm{rpm}$ ). Subsequently, the bacterial culture was cooled on ice and spun (15 min, $1500 \mathrm{rpm}, 4^{\circ} \mathrm{C}$ ). The pellet was resuspended in $34 \mathrm{ml} \mathrm{RF1}$ buffer, incubated (10 min, ice) and centrifuged ( $30 \mathrm{~min}, 1500 \mathrm{x} \mathrm{g}, 4^{\circ} \mathrm{C}$ ). The pellet was resuspended in $2 \mathrm{ml} \mathrm{RF} 2$ buffer and aliquots of $100 \mu \mathrm{l}$ were snap frozen in liquid nitrogen and stored at $-80^{\circ} \mathrm{C}$.

\subsubsection{Plasmid isolation}

The NucleoSpin Plasmid Kit was used for plasmid mini preparations. A single E. coli colony was inoculated in $3 \mathrm{ml} 2 \mathrm{YT}$ medium containing ampicillin $(100 \mu \mathrm{g} / \mathrm{ml})$ and incubated overnight $\left(37^{\circ} \mathrm{C}, 250 \mathrm{rpm}\right)$. The culture was spun down (5 min, $\left.5000 \mathrm{rpm}\right)$ and the pellet was treated according to the manufacturer's protocol. DNA was eluted with $50 \mu$ of sterile $\mathrm{H}_{2} \mathrm{O}$.

\subsubsection{Transformation}

Chemically competent bacteria are able to take up plasmids following a heat shock at $42^{\circ} \mathrm{C}$. An aliquot of competent $E$. coli cells was thawed on ice and incubated with $10 \mathrm{ng}$ plasmid DNA. Following incubation ( $45 \mathrm{~min}$, ice), a heat shock $\left(90 \mathrm{sec}, 42^{\circ} \mathrm{C}\right.$ ) was performed. After incubation on ice $(2 \mathrm{~min}$ ) the volume was adjusted to $500 \mu$ with LB medium $(399 \mu \mathrm{l})$ and incubated again $\left(2 \mathrm{~h}, 37^{\circ} \mathrm{C}\right)$. Finally, $50 \mu \mathrm{l}$ of the freshly transformed bacteria were plated out on LB agar plates containing ampicillin $(100 \mu \mathrm{g} / \mathrm{ml})$ and grown overnight at $37^{\circ} \mathrm{C}$.

\subsubsection{Protein expression and purification}

A single colony of freshly transformed E. coli BL21(DE3) was grown in a $3 \mathrm{ml}$ starter culture 2 YT medium containing ampicillin $(100 \mu \mathrm{g} / \mathrm{ml})\left(\mathrm{o} / \mathrm{n}, 37^{\circ} \mathrm{C}, 250 \mathrm{rpm}\right) .100 \mathrm{ml} 2 \mathrm{YT}$ antibiotic containing medium were inoculated with the starter culture $\left(\mathrm{OD}_{600}=0.1\right)$ and incubated until an $\mathrm{OD}_{600}$ of $0.6\left(37^{\circ} \mathrm{C}, 250 \mathrm{rpm}\right)$ was reached. Protein expression was induced by addition of IPTG (final concentration $100 \mu \mathrm{M}$ ). Following overnight 
incubation $\left(30^{\circ} \mathrm{C}, 250 \mathrm{rpm}\right)$, the culture was spun $\left(15 \mathrm{~min}, 4000 \mathrm{rpm}, 4^{\circ} \mathrm{C}\right)$ and the bacterial pellet was frozen at $-20^{\circ} \mathrm{C}$. Nanobodies were harvested by lysis of the bacteria with $5 \mathrm{ml}$ BugBugster extraction reagent supplemented with $10 \mu \mathrm{l}$ lysozyme $(10 \mathrm{mg} / \mathrm{ml})$ and $5 \mu \mathrm{l}$ benzonase $(250 \mathrm{U} / \mu \mathrm{l})$ and incubated on an overhead shaker (30 min, RT). The solution was spun (20 min, $4000 \mathrm{rpm}, 4^{\circ} \mathrm{C}$ ) and the supernatant was incubated on $500 \mu \mathrm{l}$ of a Cobalt-Resin (Talon SuperFlow; 30 min, overhead, RT). The slurry was washed twice with $5 \mathrm{ml}$ buffer WP1 and twice with $5 \mathrm{ml}$ WP2 by centrifugation $\left(2 \mathrm{~min}, 700 \times \mathrm{g}, 4^{\circ} \mathrm{C}\right.$ ) and eluted with $5 \mathrm{ml}$ EP-buffer (15 min, overhead, RT). The slurry was spun down $\left(2 \mathrm{~min}, 700 \times \mathrm{g}, 4^{\circ} \mathrm{C}\right)$ and the supernatant was transferred on a spin filter to remove the imidazole from the elution buffer and exchange the buffer to PBS. The protein in PBS was transferred in LoBind Eppendorf tubes, aliquoted and stored at $-20^{\circ} \mathrm{C}$.

\subsubsection{Cell culture}

\section{Thawing cells}

Thawing of frozen cells was performed as fast as possible to avoid the toxic side effects of dimethyl sulfoxide (DMSO) in the freezing medium. The cryo-tube was placed in the water bath at $37^{\circ} \mathrm{C}$ until a little piece of ice clot was still visible in the cryotube. The cell suspension was transferred to $10 \mathrm{ml}$ cool Dulbecco's modified eagle medium (DMEM) containing fetal calf serum (FCS; 10\%). Following centrifugation ( $2 \mathrm{~min}, 1200 \mathrm{rpm}, \mathrm{RT}$ ) the supernatant was discarded and pellet resuspended in prewarmed fresh DMEM containing $10 \%$ FCS and transferred to a $25 \mathrm{~cm}^{2}$ cell culture flask. Cells were allowed to attach overnight $\left(37^{\circ} \mathrm{C}, 5 \% \mathrm{CO}_{2}, 95 \%\right.$ humidity) and medium was changed the next day.

\section{Maintaining a cell culture}

Human mammary carcinoma cell lines MDA-MB-231 and MDA-MB-468, human epidermoid carcinoma cell line A431 and human embryonic kidney cell line HEK239 were cultured in high glucose (4.5 g/l) DMEM with Glutamax supplemented with FCS (10\%). All cells were grown in monolayer at $37^{\circ} \mathrm{C}, 5 \% \mathrm{CO}_{2}$ in a humidified atmosphere and detached with $0.05 \%$ trypsin/EDTA at a subconfluent stage. 
Cryo-storage of cells

Cell lines were frozen in liquid nitrogen for long-time storage. Cells were detached with 0.05\% trypsin/EDTA, spun down ( $2 \mathrm{~min}, 1200 \mathrm{rpm}, \mathrm{RT})$, resuspended in freezing medium and frozen as $1 \mathrm{ml}$ aliquots of $1 \times 10^{6}$ cells in cryo-tubes. They were immediately stored at $-20^{\circ} \mathrm{C}$ for $2 \mathrm{~h}$ and relocated to $-80^{\circ} \mathrm{C}$ overnight before the next day, storing them in liquid nitrogen.

\section{Transfection of eukaryotic cells}

For transfection, $6.5 \times 10^{5}$ HEK293 cells were seeded per well on a 6-well plate and allowed to attach overnight. $3 \mu \mathrm{g}$ plasmid DNA were diluted in $150 \mu \mathrm{l}$ OptiMEM and added to $150 \mu$ l Lipofectamine 2000. After incubation ( $5 \mathrm{~min}, \mathrm{RT}$ ), $250 \mu \mathrm{l}$ of the DNAlipid complex were dispersed on the cells. Protein expression was investigated after 48 to $72 \mathrm{~h}$ by flow cytometry or Western blotting.

\subsubsection{Western blot}

Cells were seeded on $6 \mathrm{~cm}$ petri dishes and allowed to grow to a subconfluent stage, washed with cold PBS and lysed with radioimmunoprecipitation assay (RIPA) buffer and scraped of the petri dish. Cell lysates were transferred to $1.5 \mathrm{ml}$ Eppendorf tubes, kept on ice for $10 \mathrm{~min}$ and spun $\left(10 \mathrm{~min}, 14000 \mathrm{rpm}, 4^{\circ} \mathrm{C}\right) .25 \mu \mathrm{g}$ of the supernatant (total lysate) were separated by SDS-PAGE either on a $3-8 \%$ or a $4-12 \%$ gel and blotted on a nitrocellulose membrane $(2 \mathrm{~h}, 50 \mathrm{~V})$. The membrane was blocked with casein $(0.1 \%)$ in TBS. Primary antibodies were diluted in casein $(0.1 \%)$ in TBS and incubated on the membrane overnight at $4^{\circ} \mathrm{C}$ with gentle agitation. The membrane was washed 3x with TBS containing Tween20 (0.05\%) for 10 min and incubated with either the secondary anti-mouse or secondary anti-rabbit antibody conjugated to a horseradish peroxidase (HRP). Secondary antibodies were diluted 1:10000 in 0.1\% casein in TBS and incubated for $1 \mathrm{~h}$ at RT with gentle agitation. The membrane was washed again and proteins were detected by brief incubation with the enhanced chemiluminescence (ECL) detection kit and by acquisition on a ChemiDoc XRS system. 


\subsubsection{Immunoprecipitation}

For immunoprecipitaion (IP), $100 \mu \mathrm{g}$ of cell lysates were diluted with $500 \mu \mathrm{P}$ PBS and incubated with $10-50 \mu \mathrm{g}$ antibodies (overnight, overhead, $4^{\circ} \mathrm{C}$ ). For IP with nanobodies, a "capture antibody", anti-c-myc was used to detect the nanobody. $15 \mu \mathrm{g}$ of anti-c-myc antibody were bound to $75 \mu$ protein $\mathrm{G}$ magnetic beads and incubated overnight. IP with full IgG antibodies did not require a capture antibody, since full IgGs bind to protein $G$. The beads were washed $3 x$ with PBS, incubated with the lysate antibody solution $\left(1 \mathrm{~h}\right.$, overhead, $\left.4^{\circ} \mathrm{C}\right)$ and washed $3 \mathrm{x}$ with PBS again. Bound proteins were eluted with $10 \mu \mathrm{l} 1 \mathrm{x}$ lithium dodecyl sulfate (LDS) sample buffer ( $10 \mathrm{~min}, 70^{\circ} \mathrm{C}$ ) and analyzed by Western blotting.

\subsubsection{Silver stain}

Silver staining is a method to visualize proteins and was performed on proteins that were separated on SDS-PAGE gels. Proteins in SDS gels were fixed by incubation in (40\% (v/v) ethanol, $10 \%(\mathrm{v} / \mathrm{v})$ acetic acid) for at least $1 \mathrm{~h}$ followed by two washing steps in $30 \%$ ethanol for $20 \mathrm{~min}$ and one washing step in deionized water for $20 \mathrm{~min}$. Gels were sanitized in $0.012 \%(\mathrm{w} / \mathrm{v})$ sodium thiosulfate $\left(\mathrm{Na}_{2} \mathrm{~S}_{2} \mathrm{O}_{3}\right)$ for $1 \mathrm{~min}$, subsequently rinsed with deionized water $(3 \mathrm{x}, 20 \mathrm{sec})$ and impregnated for $20 \mathrm{~min}$ with $0.2 \%(\mathrm{w} / \mathrm{v})$ silver nitrate $\left(\mathrm{AgNO}_{3}\right)$ and $0.05 \%(\mathrm{v} / \mathrm{v})$ paraformaldehyde (PFA) solution (37\%), that corresponds to a final concentration of $0.02 \%$. Gels were rinsed with deionized water $(3 \mathrm{x}, 20 \mathrm{sec})$ and developed for $2-10 \mathrm{~min}$ in a $3 \%(\mathrm{w} / \mathrm{v})$ sodium carbonate $\left(\mathrm{Na}_{2} \mathrm{CO}_{3}\right)$ and $0.05 \%(w / v)$ formaldehyde solution (37\%) under visual inspection. The developmental reaction was terminated by rinsing the gels in deionized water $(20 \mathrm{sec})$ and incubation in $5 \%(v / v)$ acetic acid for $10 \mathrm{~min}$. Finally, the gels were washed 3 times for $10 \mathrm{~min}$ in deionized water and scanned on a flatbed scanner (Perfection V800).

\subsubsection{Coomassie stain}

Colloidal coomassie staining is a procedure to visualize proteins in a gel. Proteins in SDS gels were fixed with $40 \%(\mathrm{v} / \mathrm{v})$ ethanol and $10 \%(\mathrm{v} / \mathrm{v})$ acetic acid for at least $60 \mathrm{~min}$ and washed twice in deionized water for $10 \mathrm{~min}$. Gels were stained overnight in the 
colloidal Coomassie staining solution and washed with $1 \%(\mathrm{v} / \mathrm{v})$ acetic acid until all Coomassie particles were removed and the background appeared clear. Finally, the gels were scanned on a flatbed scanner (Perfection V800).

\subsubsection{Flow cytometry}

Binding capacities of antibodies and nanobodies were assessed by flow cytometry. Cells were detached by brief incubation with trypsin/EDTA, washed and counted. $1 \times 10^{6}$ cells were blocked in BSA (2\%; w/v) in PBS and incubated with $100 \mu$ nanobody or antibody diluted in BSA $(2 \% ; 10 \mu \mathrm{g} / \mathrm{ml})$ for $30 \mathrm{~min}$ on ice. The nanobodies were detected by consecutive incubation with an anti-myc antibody $(4 \mu \mathrm{g} / \mathrm{ml}$; BSA $2 \%)$ (clone 9E10) and goat-anti-mouse coupled to R-Phycoerythrin (PE; $4 \mu \mathrm{g} / \mathrm{ml}$; BSA 2\%), each for $30 \mathrm{~min}$ on ice. Primary antibodies were detected by incubation with Protein $\mathrm{G}$ coupled to AlexaFluor-488 (4 $\mathrm{\mu g} / \mathrm{ml} ; 2 \% \mathrm{BSA})$ for $30 \mathrm{~min}$. Fluorescence intensities were measured by flow cytometry on a FACSAria cell sorter with FACSDiva software. Data were analyzed using FlowJo software (v. 7.6.5).

\subsubsection{Surface plasmon resonance}

The affinity and the association and dissociation constants of the anti-Kv10.1 nanobodies to $\mathrm{H} 1 \mathrm{X}$ were determined by surface plasmon resonance (SPR) in a Biacore2000 machine. In a first step, the ligand H1X was labeled on a CM5 chip according to manufacturer's guide at pH 4.5 on flow cell 2 . Nanobodies were diluted in buffer HBS-EP to concentrations ranging from $3.1 \mathrm{nM}$ to $400 \mathrm{nM}$ and injected on the flow cell for 2 min with a flow rate of $30 \mu$ per min. Binding or rather association ( $2 \mathrm{~min}$ ) of the nanobodies and dissociation $(4 \mathrm{~min}$ ) was recorded for flow cell 1 and 2 followed by two regeneration cycles with glycine $(10 \mathrm{mM}, \mathrm{pH} 2.5)$ to remove remaining nanobodies from the surface of the CM5 chip. The BIAevaluation software (v. 4.1.1) was used for analysis. Sensograms of flow cell 1 (background) were subtracted from flow cell 2, normalized and fitted to a 1:1 Langmuir model for determination of affinity, association and dissociation constants. 


\subsubsection{Immunohistochemistry}

Dissected tumors or organs were fixed in a 4\% PFA / PBS solution (>24 h, RT) and transferred to tissue embedding cassettes. The tissues were dehydrated in an ethanol series (Table 14) in a benchtop tissue processor (TP1020) and embedded in paraffin.

Table 14: Dehydration scheme for IHC

\begin{tabular}{cc}
\hline Time & Solvent \\
\hline $3 \times 60 \mathrm{~min}$ & $75 \%$ ethanol \\
$2 \times 90 \mathrm{~min}$ & $96 \%$ ethanol \\
$3 \times 75 \mathrm{~min}$ & $100 \%$ ethanol \\
$2 \times 120 \mathrm{~min}$ & $100 \% \times y l o l$ \\
\hline
\end{tabular}

Paraffin tissue sections ( 2 or $5 \mu \mathrm{m}$ thick) were deparaffinized twice in xylene for $20 \mathrm{~min}$ each, rehydrated in an ethanol series (100\%, 95\%, 70\%, 50\%, 30\%) for 5 min each and washed twice in TBS for $5 \mathrm{~min}$. Antigen retrieval was performed by incubation in Antigen-retrieval buffer at $90^{\circ} \mathrm{C}$ in a steamer for $10 \mathrm{~min}$. Slides were allowed to cool down to RT in the buffer for $20 \mathrm{~min}$ and washed for $5 \mathrm{~min}$ in TBS. The endogenous peroxidase was inhibited by incubation in peroxidase blocking solution for $10 \mathrm{~min}$ and subsequent washing (2x $5 \mathrm{~min}$ ) in TBS. Non-specific binding sites were blocked by incubation in Seablock buffer (containing steelhead salmon serum) in TBS for 20 min at RT. For detection of human EGFR, the primary anti-human EGFR antibody (clone SP9) was diluted 1:400 in antibody diluent and incubated on the slides overnight at $4^{\circ} \mathrm{C}$. The slides were washed twice in TBS for 5 min before one drop of Histofine Simple Stain Max PO, containing an anti-rabbit-HRP antibody, was added at RT for $1 \mathrm{~h}$. Slides were washed with TBS for 5 min and antibodies were detected by incubation with ImmPACT 3,3'-Diaminobenzidine (DAB) Peroxidase substrate for $30 \mathrm{~min}$. Slides were washed again for $5 \mathrm{~min}$ in TBS, counterstained with Hematoxylin for $20 \mathrm{sec}$ and washed twice with deionized water for 2 min.

Following counterstaining the sections were dehydrated in the ethanol series in reversed order $(30 \%, 50 \%, 70 \%, 90 \%, 100 \%)$ for $5 \mathrm{~min}$ each and cleared in xylene twice for $5 \mathrm{~min}$. The sections were mounted with Cytoseal60 and dried overnight at $4^{\circ} \mathrm{C}$. 


\subsubsection{Radiolabeling of antibodies}

For radiolabeling of the full $\operatorname{lgG}_{1}$ antibodies, the free $\varepsilon$-amino groups were modified with 6-hydrazinonicotinamide (HyNic). $10 \mathrm{nmol}$ antibody were incubated with an 30 molar excess of succinimidyl 6-hydraziniumnicotinate hydrochlorate (SHNH) for $2 \mathrm{~h}$ at ambient temperature in modification buffer. $20 \mu \mathrm{l}$ of the antibody-HYNIC conjugate $\left(2 \mathrm{mg} / \mathrm{ml}\right.$ ) in conjugation buffer were incubated with $500 \mu \mathrm{l}^{99 \mathrm{~m}} \mathrm{Tc}$ pertechnetate (1000$2000 \mathrm{MBq})$, tin chloride $\left(\mathrm{SnCl}_{2} ; 5 \mu \mathrm{l} ; 1 \mathrm{mg} / \mathrm{ml}\right)$ and tricine $(50 \mu \mathrm{l} ; 100 \mathrm{mg} / \mathrm{ml})$ as coligand for $20 \mathrm{~min}$ at ambient temperature. The radiolabeled antibodies were purified from unbound activity on Amicon Ultra centrifugal filters. The radiochemical yield and purity of the radiolabeling procedures were determined by instant thin layer chromatography (ITLC) on silica gel (ITLC-SG) strips and $1 \% \mathrm{HCl}$ in methanol as mobile phase.

\subsubsection{Radiolabeling of nanobodies}

The nanobodies were labeled with technetium-99m tricarbonyl $\left(\left[{ }^{99 m} \mathrm{Tc}(\mathrm{CO})_{3}\right]^{+}\right)$species via their C-terminal hexahistidine tags (6xHis). The CRS Kit (Paul Scherrer Institut) was used to produce the $\left[{ }^{99 \mathrm{~m}} \mathrm{Tc}(\mathrm{CO})_{3}\right]^{+}$intermediate out of $2400-4000 \mathrm{MBq}$ of ${ }^{99 \mathrm{~m}} \mathrm{Tc}$ pertechnetate in $1 \mathrm{ml}$ saline. After boiling for $20 \mathrm{~min}$ at $100^{\circ} \mathrm{C}, 500 \mu \mathrm{l}$ of $\left[{ }^{99 \mathrm{~m}} \mathrm{Tc}(\mathrm{CO})_{3}\right]^{+}$ were neutralized with $1 \mathrm{M} \mathrm{HCl}$ to $\mathrm{pH} 7.5$ and incubated with $50 \mu \mathrm{g}$ nanobody $(1 \mathrm{mg} / \mathrm{ml})$ for $90 \mathrm{~min}$ at $56^{\circ} \mathrm{C}$. The radiolabeled nanobodies were purified from unbound activity on Amicon Ultra centrifugal filters. The radiochemical yield and purity were determined as described above.

\subsubsection{Serum stability studies}

Nanobodies ${ }^{99 \mathrm{~m}} \mathrm{Tc}$-D10 and ${ }^{99 \mathrm{~m}} \mathrm{Tc}-\mathrm{F} 5$ and $\operatorname{lgG}_{1}{ }^{99 \mathrm{~m}} \mathrm{Tc}$-Cetuximab were labeled with ${ }^{99 \mathrm{~m}} \mathrm{Tc}$ as described above. 2 - $3 \mu \mathrm{g}$ of the radiolabeled proteins were mixed with mouse serum and incubated at $37^{\circ} \mathrm{C}$. Samples were taken at 0, 30, 60, 120 and $240 \mathrm{~min}$ and analyzed by thin layer chromatography using ITLC-SG strips and $1 \% \mathrm{HCl}$ in methanol as mobile phase. 


\subsubsection{Tumor mouse models}

All animal experiments were carried out according to the German animal welfare law and were approved by local authorities (33.9-42502-04-12/0701). Cells in exponential growth phase (before reaching a subconfluent stage) that were intended for implantation were rinsed with PBS, detached with EDTA (5 mM) and collected with $10 \mathrm{ml}$ warm culture medium. The cell suspension was spun ( $2 \mathrm{~min}, 1200, \mathrm{rpm}, \mathrm{RT}$ ) and the pellet was washed twice and resuspended in PBS. Cells were counted in a counting chamber using trypan blue.

Cells of the human epidermoid carcinoma cell line A431 (1.5 x 10 in $100 \mu \mathrm{lPBS})$ were injected with an insulin syringe $(30 \mathrm{G})$ subcutaneously in the right flank of 8 - 12 weeks old male athymic nude mice under control of $2 \%$ isoflurane. Cells of the human mammary carcinoma cell lines MDA-MB-231 ( 1 × 10 in $20 \mu \mathrm{IPBS})$ and MDA-MB-468 (2 $\times 10^{6}$ in $20 \mu$ PBS) were implanted orthotopically in the fat pad of the right abdominal mammary gland of $8-12$ weeks old female athymic nude mice under the control of a general Ketamine/Rompun anesthesia (intraperitoneal (i.p.) injection; Ketamine $10 \%$ (90 $\mu \mathrm{g} / \mathrm{g}$ body weight) and Rompun 2\% (7.5\%/g body weight). A small incision was made approximately $1 \mathrm{~cm}$ underneath the right abdominal mammary gland. The fad pad was pulled out carefully and the cells were injected in a volume of $20 \mu \mathrm{l}$ PBS with an insulin syringe (30G). The incision was closed by $2-3$ surgical stitches with surgical sutures (4/0). Mice were kept on a heating plate at $37^{\circ} \mathrm{C}$ and monitored until recovery from anesthesia. After recovery, the mice were placed in their cages and put back on the housing racks. Mice were housed in individually ventilated cages in the central animal facility of the University Medical Center Göttingen. Food and water were given ad libitum. Tumor growth and health conditions were monitored twice a week by palpation and visual inspection, respectively.

\subsubsection{In vivo $\mathrm{CT}$}

Most tumor volumes were determined in vivo by contrast-enhanced computed tomography (CT) scans under inhalation anesthesia (1\% isoflurane). $100 \mu$ l Ultravist370 were injected i.v. and two $360^{\circ}$ scans were carried out on a low-dose in vivo small animal CT (Quantum FX) with the following parameter: $90 \mathrm{kV}, 200 \mu \mathrm{A}$ and a field of 
view of 40 ( 2 min total scan time; 1 min post injection) and $73 \mathrm{~mm}$ (17 sec total scan time; 5 min post injection). Image reconstruction was performed using a standard filtered backprojection algorithm implemented in the vendors software resulting in data sets with a voxel size of either $\sim 80 \times 80 \times 80 \mu \mathrm{m}^{3}$ or $140 \times 140 \times 140 \mu^{3}$.

\subsubsection{In vivo SPECT imaging}

SPECT was performed on a triple-head clinical gamma camera equipped with multipinhole collimators with 6 pinholes and a field of view of $50 \times 60 \mathrm{~mm}$ (HiSPECT) on tumor bearing mice under $1 \%$ isoflurane anesthesia. SPECT scans of mice that received radiolabeled nanobodies were performed 45 min post i.v. injection of ${ }^{99 \mathrm{~m}} \mathrm{Tc}-\mathrm{F} 5$ and ${ }^{99 \mathrm{~m}} \mathrm{Tc}$-D10 in the tail vein. SPECT scans of mice, that received radiolabeled ${ }^{99 \mathrm{~m}} \mathrm{Tc}$ Cetuximab, were started $45 \mathrm{~min}$ and/or $24 \mathrm{~h}$ post i.v. injection. Images were acquired over $360^{\circ}$ in 10 projections of 300 sec each into $256 \times 256$ matrices, resulting in a total scan time of $50.6 \mathrm{~min}$. SPECT scans with the anti-EGFR nanobody ${ }^{99 \mathrm{~m}} \mathrm{Tc}-\mathrm{D} 10$ and the control nanobody ${ }^{99 \mathrm{~m}} \mathrm{Tc}-\mathrm{F} 5$ were performed in the same animals using identical scan protocols. ${ }^{99 \mathrm{~m}} \mathrm{Tc}-\mathrm{D} 10$ was injected i.v. at least $72 \mathrm{~h}$ after administration of the control nanobody ${ }^{99 m}$ Tc-F5. In analogy to the nanobodies SPECT scans with the anti-EGFR antibody ${ }^{99 m} \mathrm{Tc}$-Cetuximab and the isotype ${ }^{99 \mathrm{~m}} \mathrm{Tc}-\lg G_{1}$ were performed in the same animals using identical scan protocols as well. ${ }^{99 \mathrm{~m}} \mathrm{Tc}$-Cetuximab was injected i.v. at least $48 \mathrm{~h}$ after administration of the isotype ${ }^{99 \mathrm{~m}} \mathrm{Tc}-\operatorname{lgG}_{1}$

Image reconstruction was performed using an ordered-subset expectation maximization algorithm implemented in the HiSPECT software (SciVis) resulting in data sets with a voxel size of $600 \times 600 \times 600 \mu^{3}$. Since CT and SPECT scans were performed on separate systems on different days, each mouse was imaged in the same animal holders, which included 6 holes each filled with ${ }^{99 m}$ Tc-pertechnetate (<30 kBq) that were used for manual alignment.

\subsubsection{Ex vivo biodistribution analysis}

Following SPECT scans, mice were sacrificed by cervical dislocation under isoflurane anesthesia. Different organs and the tumor were dissected, blotted dry, weighed and 
transferred to counting tubes. Remaining activities in organs and tumors were measured in a gamma counter (Wallac Wizard 3" 1480 automatic gamma counter), decay corrected and expressed as percent injected dose per gram (\% ID/g). The total injected dose of each mouse was determined by measuring the activity in the syringes before and after injection. The tumors were stored in a PFA (4\%; v/v)) / PBS solution after measurement of the remaining activity and kept for subsequent IHC in the Department of Nuclear Medicine (University Medical Center Göttingen) until radioactivity was cooled-down.

\subsubsection{Blood clearance}

The blood clearance and serum half-life of radiolabeled imaging probes were investigated in non-tumor bearing mice at various time points $(1,5,10,20,40,60,90$, $120,240,1200,1440$ and $1560 \mathrm{~min}$ ) post i.v. probe injection by retrobulbar blood withdrawal. Approx. 2 - 3 drops of blood were sampled, weighed and measured in a gamma counter (Wallac Wizard 3" 1480 automatic gamma counter), decay corrected and expressed as percent injected dose per gram (\% ID/g). Serum half-life and plateau were determined by non-linear regression applying a of a one phase exponential decay fitting.

\subsubsection{Image data anaylsis}

Reconstructed CT and SPECT data sets were displayed, quantified and analyzed for tumor volume as well as for tumor and unspecific tissue uptake of radiolabeled probes using Scry v. 5.0. SPECT data were decay corrected and normalized. All tumor volumes were segmented from contrast enhanced CT and SPECT images by a region growing algorithm. For reference a region with the same size like the tumor containing tissue only was segmented on the contra lateral side and was used for determination of unspecific tissue uptake and finally for determination of in vivo tumor to tissue ratio. Tumor and tissue uptake were expressed as percentage of injected dose per cubic centimeter $\left(\% \mathrm{ID} / \mathrm{cm}^{3}\right)$. 


\subsubsection{Statistical analyis}

Statistical analysis was performed using GraphPad Prism v. 6.01 for Windows. Paired or unpaired two-tailed t-Tests (indicated in the analysis) were used to determine the significant difference between the groups in ex vivo and in vivo comparison. Unless otherwise stated all values were calculated as mean \pm standard deviation (SD). 


\section{Results}

\subsection{Generation of anti-Kv10.1 nanobodies}

To enable specific tumor visualization with anti-Kv10.1 nanobodies using in vivo SPECT imaging, anti-Kv10.1 nanobodies had to be generated first. Therefore, a llama was immunized twice with the antigen $\mathrm{H} 1 \mathrm{X}$, a fusion protein consisting of thioredoxin and two parts of the E3 loop of Kv10.1 ${ }^{1}$. The resulting antibody library with a diversity of $1.3 \times 10^{7}$ clones was used for selection and screening of the anti-Kv10.1 nanobodies and the lead candidates were characterized for specific binding to the ion channel Kv10.1.

\subsubsection{Selection of ten anti-Kv10.1 nanobodies}

Phage display

The selection of anti-Kv10.1 nanobodies was performed by phage display. In order to generate an initial phage library, the antigen library was infected with the helper phage KM13. Depending on the selection strategy of each round, phages were depleted on immobilized TRX and incubated with different concentrations of immobilized H1X before washing thoroughly to remove unbound phages (Table 15). To determine the selection efficiency, the number of colony formation units (CFU) of E. coli infected with an aliquot of the phages was determined before (input) and after selection (output) (Table 15). In total, 9 rounds of selection were performed yielding a reduction of approximately three orders of magnitude per selection round resulting in an enrichment of binding phages in each selection.

\footnotetext{
${ }^{1}$ Immunization and establishment of the antibody library was performed from the lab staff of Daniel Baty and Patrick Chames at INSERM, Marseille, France.
} 
Table 15: Parameters of nanobody selection

\begin{tabular}{|c|c|c|c|c|}
\hline $\begin{array}{l}\text { Round of } \\
\text { Selection }\end{array}$ & $\begin{array}{l}\text { Phages of } \\
\text { selection }\end{array}$ & $\begin{array}{c}\text { Target } \\
\text { selection }\end{array}$ & Input (CFU) & Output (CFU) \\
\hline 1 & Phage library & $20 \mu \mathrm{M} \mathrm{H} 1 \mathrm{X}$ & $6.0 \times 10^{11}$ & $3.0 \times 10^{8}$ \\
\hline 2 & 1 & $20 \mu \mathrm{M} \mathrm{H} 1 \mathrm{X}$ & $2.0 \times 10^{10}$ & $3.5 \times 10^{8}$ \\
\hline 3 & 2 & $\begin{array}{l}\text { depletion on } \\
20 \mu \mathrm{M} \text { TRX; } \\
20 \mu \mathrm{M} \text { H1X }\end{array}$ & $4.0 \times 10^{12}$ & $4.0 \times 10^{8}$ \\
\hline 4 & 3 & $\begin{array}{l}\text { depletion on } \\
20 \mu \mathrm{M} \text { TRX; } \\
20 \mu \mathrm{M} \text { H1X }\end{array}$ & $6.0 \times 10^{12}$ & $3.0 \times 10^{9}$ \\
\hline 5 & $1+2$ & 100 nM H1X & $3.4 \times 10^{12}$ & $1.4 \times 10^{10}$ \\
\hline 6 & Phage library & $\begin{array}{l}\text { depletion on } \\
1.8 \mu \mathrm{M} \text { TRX; } \\
1.1 \mu \mathrm{M} \mathrm{H} 1 \mathrm{X}\end{array}$ & $7.0 \times 10^{11}$ & $7.0 \times 10^{7}$ \\
\hline 7 & 6 & $\begin{array}{l}\text { depletion on } \\
1.8 \mu \mathrm{M} \text { TRX; } \\
1.1 \mu \mathrm{M} \mathrm{H} 1 \mathrm{X}\end{array}$ & $1.0 \times 10^{13}$ & $1.0 \times 10^{11}$ \\
\hline 8 & 7 & $\begin{array}{l}\text { depletion on } \\
1.8 \mu \mathrm{M} \text { TRX; } \\
1.1 \mu \mathrm{M} \mathrm{H} 1 \mathrm{X}\end{array}$ & $9.0 \times 10^{12}$ & $2.0 \times 10^{11}$ \\
\hline 9 & 8 & $\begin{array}{l}\text { depletion on } \\
1.8 \mu \mathrm{M} \text { TRX; } \\
5.5 \mathrm{nM} \mathrm{H} 1 \mathrm{X}\end{array}$ & $6.0 \times 10^{11}$ & $3.0 \times 10^{8}$ \\
\hline
\end{tabular}

\section{Screening}

For screening, 186 clones per selection were picked from the output plates and induced for nanobody production by addition of IPTG. The resulting nanobodies were screened on the recombinant proteins $\mathrm{H} 1 \mathrm{X}$ as a positive and TRX as a negative control by ELISA yielding more than 30 hits in total. Ten clones showing the highest signals were chosen as lead candidates and produced in higher quantity in $100 \mathrm{ml}$ cultures for further investigation. The produced anti-Kv10.1 nanobodies were isolated, blotted on a nitrocellulose membrane and detected with an anti-His antibody (Figure 10). Nanobodies were expressed mainly as monomers with a molecular weight ranging from 14 - $17 \mathrm{kDa}$. Little portions of each nanobody were expressed as multimers (faint bands) characterized by multiple molecular weights of the monomers. 


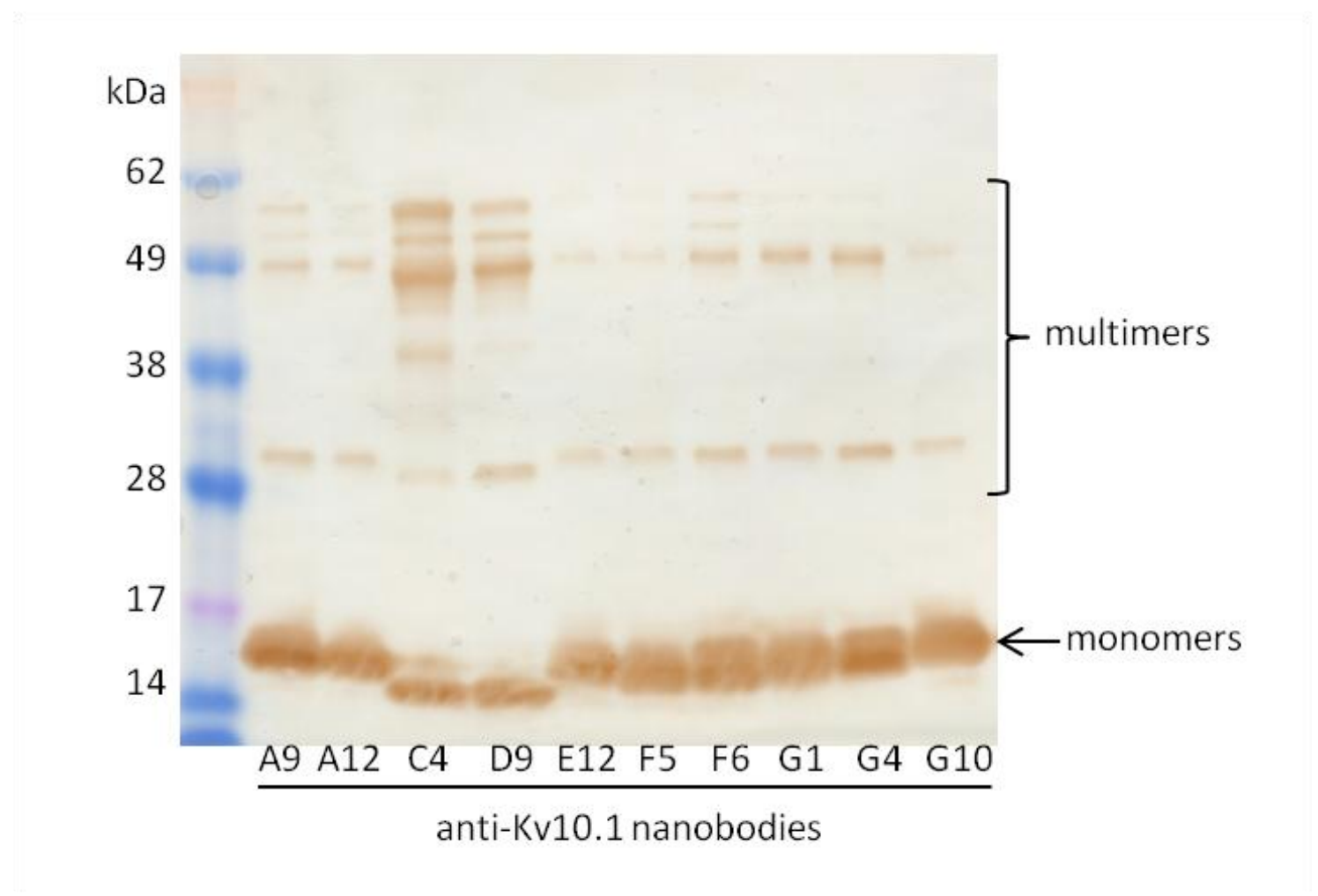

Figure 10: Western blot of anti-Kv10.1 lead candidates

Purity and size of selected anti-Kv10.1 nanobodies lead candidates were validated by Western blot. Nanobodies were expressed in $E$. coli $\mathrm{TG}_{\mathrm{TR}}$. Three $\mu \mathrm{g}$ of the purified nanobodies were separated by SDS-PAGE. The blotted proteins were revealed with a HRP conjugated anti-His antibody. The nanobodies were expressed mainly as monomers with a molecular weight ranging from 14 - $17 \mathrm{kDa}$ (arrow). To a minor extend, each nanobody was expressed as di-, tri or tetramer shown as faint bands with multiple molecular weights above $28 \mathrm{kDa}$ of the monomers. Notably, nanobodies C4 and D9 had a lower molecular weight compared to all other nanobodies.

\section{Sequencing}

E. coli clones bearing the DNA of the lead candidate nanobodies were sent to GATC Biotech (Konstanz, Germany) for sequencing. The DNA sequence was translated into amino acid code and transferred to the international immunogenetics information system (IMGT) file format. Sequencing identified 9 unique nanobodies separated in two families with pronounced differences in complementary determining regions (CDR) 2 and 3. Nanobodies C4 and D9 shared a short CDR 3 and are characterized by a lower molecular weight compared to all other tested clones (Table 16). The framework (FR) regions were conserved throughout both families. 
Table 16: Identification of nine unique anti-Kv10.1 nanobodies by sequencing

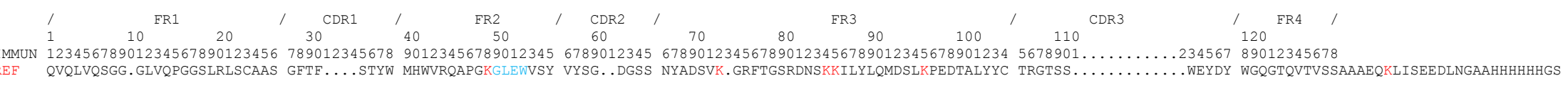

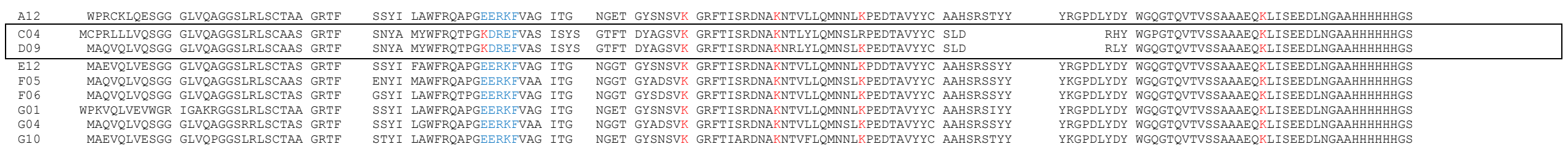

Protein sequences of nine anti-Kv10.1 nanobodies are shown in the IGMT file format. Nine unique clones were identified belonging to two different families with pronounced

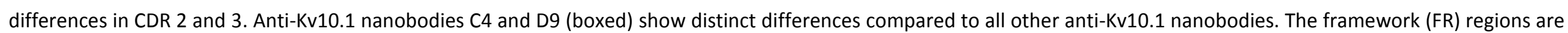

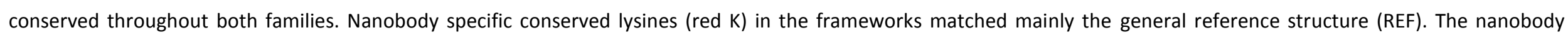
specific KGLEW pattern (light blue) in FR2 is not conserved throughout the anti-Kv10.1 nanobodies (blue). 


\section{Determination of the equilibrium binding constant $K_{d}$}

In order to estimate the binding quality of eight anti-Kv10.1 nanobodies (A9, A12, C4, D9, F5, F6, G1, G4), an ELISA plate was coated with H1X and incubated with decreasing nanobody concentrations ranging from $30 \mu \mathrm{M}$ to $0.5 \mathrm{nM}$ (Figure 11A). By applying nonlinear regression with a one-site total binding model the equlibrium binding constant $K_{d}$ was determined, i.e. the concentration needed to achieve half-maximum binding at equilibrium. Figure 11B depicts the fitting of the applied model and revealed $K_{d}$ values ranging between 10 and $942 \mathrm{nM}$. Interestingly, nanobodies C4 and D9, having a different CDR compared to all other nanobodies, had the best $K_{d}$ of $86 \mathrm{nM}$ and $10 \mathrm{nM}$, respectively compared to $K_{d}$ values up to $942 \mathrm{nM}$ of $\mathrm{G} 4$ that lies one order of magnitude higher.

A

$\mathrm{Kd}$

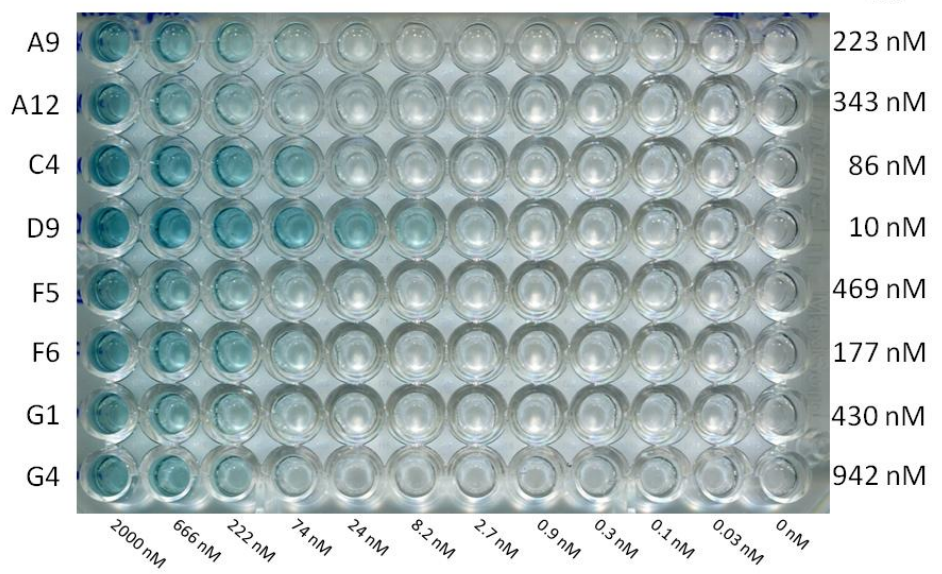

B

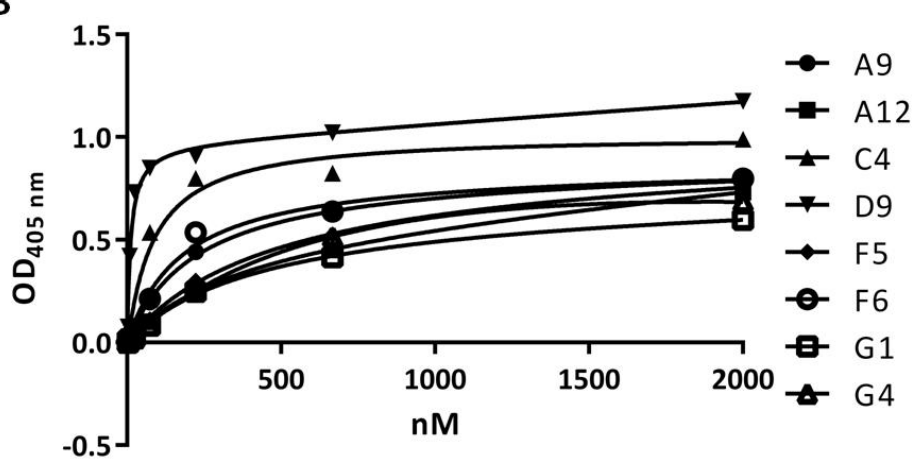

Figure 11: Binding capacities of anti-Kv10.1 nanobodies

The equilibrium binding constants $K_{d}$ of anti-Kv10.1 nanobodies were determined by ELISA. (A) Different anti-Kv10.1 nanobodies were titrated from $30 \mu \mathrm{M}$ to $0.5 \mathrm{nM}$ and incubated on immobilized H1X on ELISA plates. (B) A nonlinear regression analysis with a one-site total binding model was applied to determine $K_{d}$ values that ranged between 10 and $942 \mathrm{nM}$. Interestingly, nanobodies $C 4$ and D9 had the lowest and best $K_{d}$ values compared to all other anti-Kv10.1 nanobodies tested. 
Determination of the affinity $K_{D}$ by surface plasmon resonance analysis

Since nanobodies C4 and D9 showed the best $K_{d}$ values, they were further analyzed by in-source-decay (ICD) MS for determination of their actual molecular weights². Molecular weights of $15117 \mathrm{Da}$ and 15167 Da were identified for nanobodies C4 and D9, respectively.

The nanobodies were further analyzed for their affinity $\left(K_{D}\right)$, association $\left(k_{a}\right)$ and their dissociation $\left(k_{d}\right)$ constants by SPR. In contrast to the $K_{d}$ value, which represents the binding only in equilibrium, the affinity $K_{D}$ is the ratio of dissociation to association and depicts the actual binding. Therefore, nanobodies D9 and C4 were diluted to concentrations ranging from $3.12 \mathrm{nM}$ to $200 \mathrm{nM}$ and $25 \mathrm{nM}$ to $400 \mathrm{nM}$, respectively. Sensograms were recorded for binding of the nanobodies on immobilized H1X (Figure 12). Affinities of $1.7 \mu \mathrm{M}$ and $77 \mathrm{nM}$ for $\mathrm{C} 4$ and $\mathrm{D} 9$, respectively, were determined by using a 1:1 Langmuir model (Table 17) resulting in comparable $K_{d}$ values as previously determined for D9 (Figure 11A). Interestingly, C4 is characterized by a slower association and dissociation constant compared to D9.
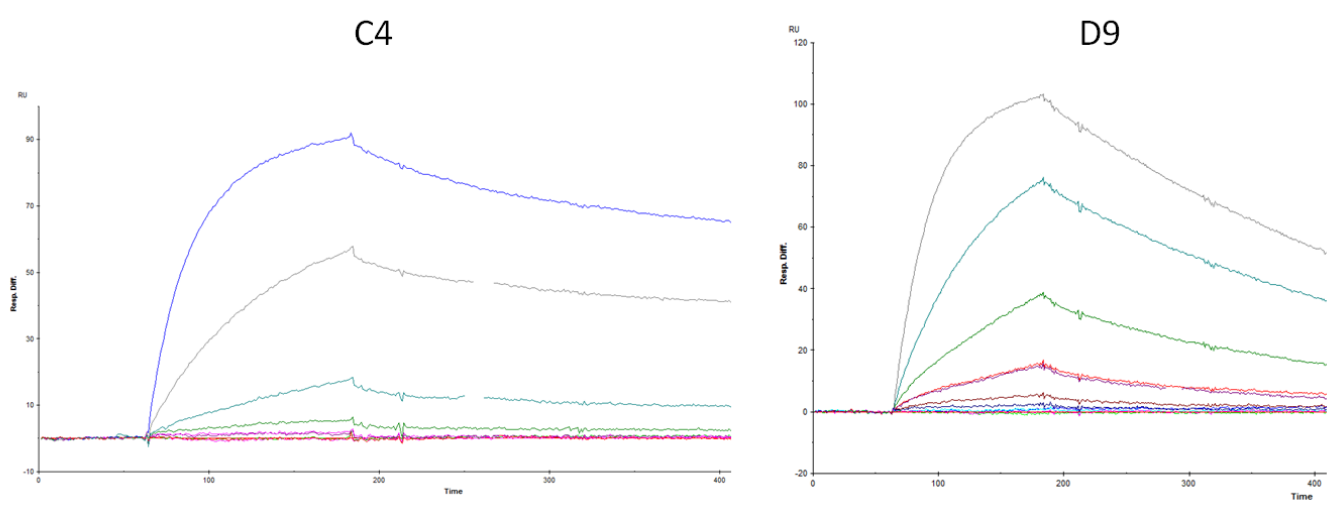

Figure 12: Sensograms of anti-Kv10.1 nanobodies C4 and D9

Affinity $\left(K_{D}\right)$, association $\left(k_{a}\right)$ and dissociation $\left(k_{d}\right)$ constants were determined for anti-Kv10.1 nanobodies $C 4$ and D9 by SPR. Nanobodies were diluted to concentrations ranging from $3.12 \mathrm{nM}$ (light blue) to $200 \mathrm{nM}$ (gray line) and from $25 \mathrm{nM}$ (purple and red line) to $400 \mathrm{nM}$ (blue line) for D9 and C4, respectively. Sensograms were recorded for binding of nanobodies D9 and C4 on immobilized H1X. Affinities of $1.7 \mu \mathrm{M}$ and $77 \mathrm{nM}$ for $\mathrm{C} 4$ and $\mathrm{D}$, respectively were determined by applying a 1:1 Langmuir model.

\footnotetext{
${ }^{2}$ ICD MS was performed by Thomas Liepold and PD. Dr. Olaf Jahn.
} 
Table 17: Affinity constants of nanobodies C4 and D9

\begin{tabular}{lccc}
\hline & $\mathbf{k}_{\mathrm{a}}(\mathbf{1} / \mathbf{M s})$ & $\mathbf{k}_{\mathrm{d}}(\mathbf{1} / \mathbf{s})$ & $\mathrm{K}_{\mathrm{D}}(\mathbf{M})$ \\
\hline C4 & 561 & $9.99 \times 10^{-4}$ & $1.78 \times 10^{-6}$ \\
D9 & $3.83 \times 10^{4}$ & $2.98 \times 10^{-3}$ & $7.78 \times 10^{-8}$ \\
\hline
\end{tabular}

\subsubsection{The anti-Kv10.1 nanobodies detect Kv10.1}

\section{Specific detection of fusion protein H1X by ELISA and Western blot}

The specificities towards Kv10.1 of each of the expressed anti-Kv10.1 nanobody lead candidates were assessed by ELISA on immobilized recombinant proteins H1X and TRX. The capacity of all produced anti-Kv10.1 nanobodies to bind the fusion protein H1X but not to the negative control TRX proved the specificity of all nanobodies towards KV10.1 (Figure 13). Staining with the substrate ABTS showed the different amounts of converted substrate. Since the same protein concentrations of $\mathrm{H} 1 \mathrm{X}(10 \mu \mathrm{g} / \mathrm{ml})$ and anti-Kv10.1 nanobodies $(10 \mu \mathrm{g} / \mathrm{ml})$ were used, a more intense staining as obtained with nanobodies C4 and D9 suggests a higher affinity towards H1X compared to all other tested nanobodies that only yielded a light blue staining.

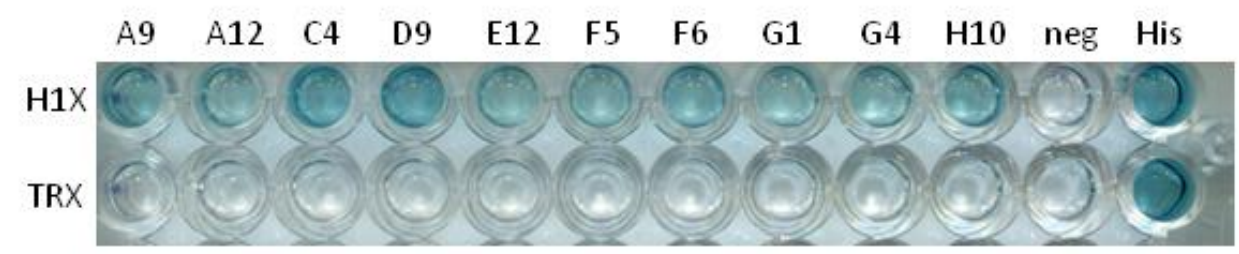

Figure 13: ELISA of anti-Kv10.1 nanobody lead candidates

The specificities towards Kv10.1 of each of the expressed anti-Kv10.1 nanobodies were validated by ELISA. All investigated nanobodies detected the fusion protein H1X but not the negative control TRX. Nanobodies were revealed by consecutive incubation with anti-myc and anti-mouse-HRP antibodies. Negative controls (neg) consisted of immobilized H1X or TRX without incubation of a nanobody. The loading control (His) proved an even coating of the wells with H1X and TRX and was revealed with the anti-His-HRP antibody. 
Western blot experiments were carried out in order to evaluate the ability of Kv10.1 nanobodies (A12, C4, D9 and G1) to bind Kv10.1 in different conformations, using the purified recombinant fusion protein $\mathrm{H} 1 \mathrm{X}$ and pCDNA-3-Kv10.1-venus transfected HEK cell lysates as well as TRX and pCDNA-3- $\varnothing$-venus transfected HEK cell lysates as negative controls (Figure 14). All tested anti-Kv10.1 nanobodies detected H1X with a molecular weight of approximately $25 \mathrm{kDa}$ on the membrane but not TRX (approx. $20 \mathrm{kDa}$ ). Kv10.1 could not be detected in HEK transfected cell lysates at approx. $110 \mathrm{kDa}$ with any of the tested anti-Kv10.1 nanobodies and anti-Kv10.1 antibody 9391 suggesting a poor transfection efficiency and low Kv10.1 expression in these cells (data not shown).

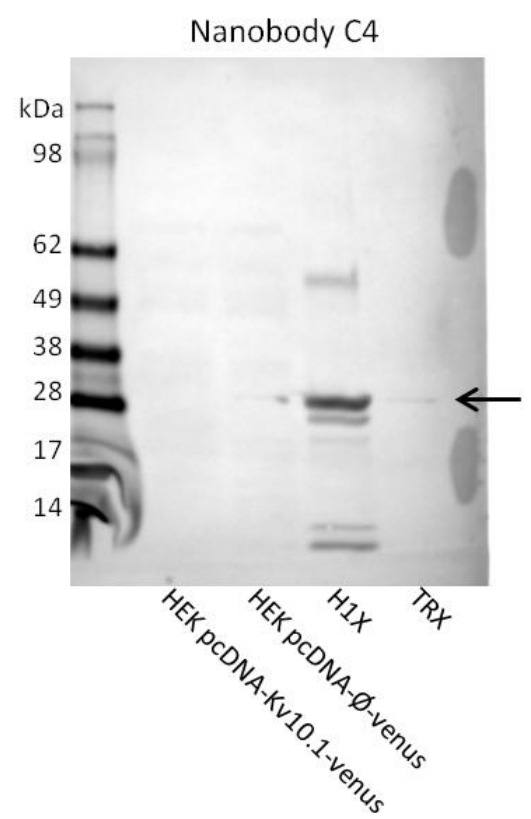

\section{Figure 14: Specific detection of blotted H1X by nanobody C4} Representative Western blot of the detection of Kv10.1 by anti-Kv10.1 nanobody C4. Whole cell lysates of pcDNA3Kv10.1-venus and pcDNA3- $\varnothing$-venus transfected HEK cells $(30 \mu \mathrm{g})$ as well as recombinant protein $\mathrm{H} 1 \mathrm{X}$ and TRX $(5 \mu \mathrm{g})$ were transferred on a nitrocellulose membrane. Incubation with anti-Kv10.1 nanobody $\mathrm{C} 4$ and detection with consecutive incubation of anti-myc and anti-mouse HRP antibodies revealed a specific protein band of approx. $28 \mathrm{kDa}$ of H1X (indicated by arrow) but not of TRX (approx. $20 \mathrm{kDa}$ ). A detection of Kv10.1 by nanobody C4 in pcDNA3-Kv10.1venus transfected HEK cell lysates was not possible. 


\section{Detection of the ion channel Kv10.1 by immunoprecipitation}

The anti-Kv10.1 nanobodies are able to detect the fusion protein $\mathrm{H} 1 \mathrm{X}$ as shown in various experiments (Figure 13 and Figure 14). However, the intention of generating a novel anti-Kv10.1 nanobody was the feasibility to detect the potassium channel Kv10.1 in cells as tumor associated protein for tumor monitoring. As a next step, the ability of binding of anti-Kv10.1 nanobodies to the native Kv10.1 potassium channel was assessed by immunoprecipitation (IP). Thus, the anti-Kv10.1 nanobodies were immobilized on magnetic beads and incubated with pTracer-Kv10.1 transfected HEK cell lysates (Figure 15). For control, pTracer- $\varnothing$ transfected HEK cells were used. For reference, in house produced and validated mouse monoclonal anti-Kv10.1 antibody 33 was applied for IP. Following separation by SDS-PAGE and Western blotting, the precipitated Kv10.1 was detected by incubation with the rabbit polyclonal anti-Kv10.1 antibody 9391. IP using either nanobody C4 or D9 resulted in a clear band at approx. $110 \mathrm{kDa}$ in the pTracer-Kv10.1 transfected HEK lysate but not in the negative control pTracer- $\varnothing$ transfected HEK lysate with both nanobodies (Figure 15). Reference antiKv10.1 antibody 33 showed bands of the same size as obtained with the nanobodies C4 (Figure 15) and D9 (data not shown) assuming a successful enrichment of Kv10.1. Compared to anti-Kv10.1 nanobodies C4 and D9, nanobody A12 was not capable to precipitate Kv10.1. For illustration of the overall transfection efficiency, the cell lysates of pTracer-Kv10.1 and pTracer- $\varnothing$ transfected HEK cells as well as the lysates of wild type (wt) HEK cells were loaded on the same gel and were detected with the polyclonal Kv10.1 antibody 9391. Only a faint band at the same size as with antibody 33 and nanobody C4 could be identified in the pTracer-Kv10.1 but not in the pTracer- $\varnothing$ transfected HEK cell lysate or in wt lysates showing again a low transfection efficiency of Kv10.1-transfected HEK cells. 


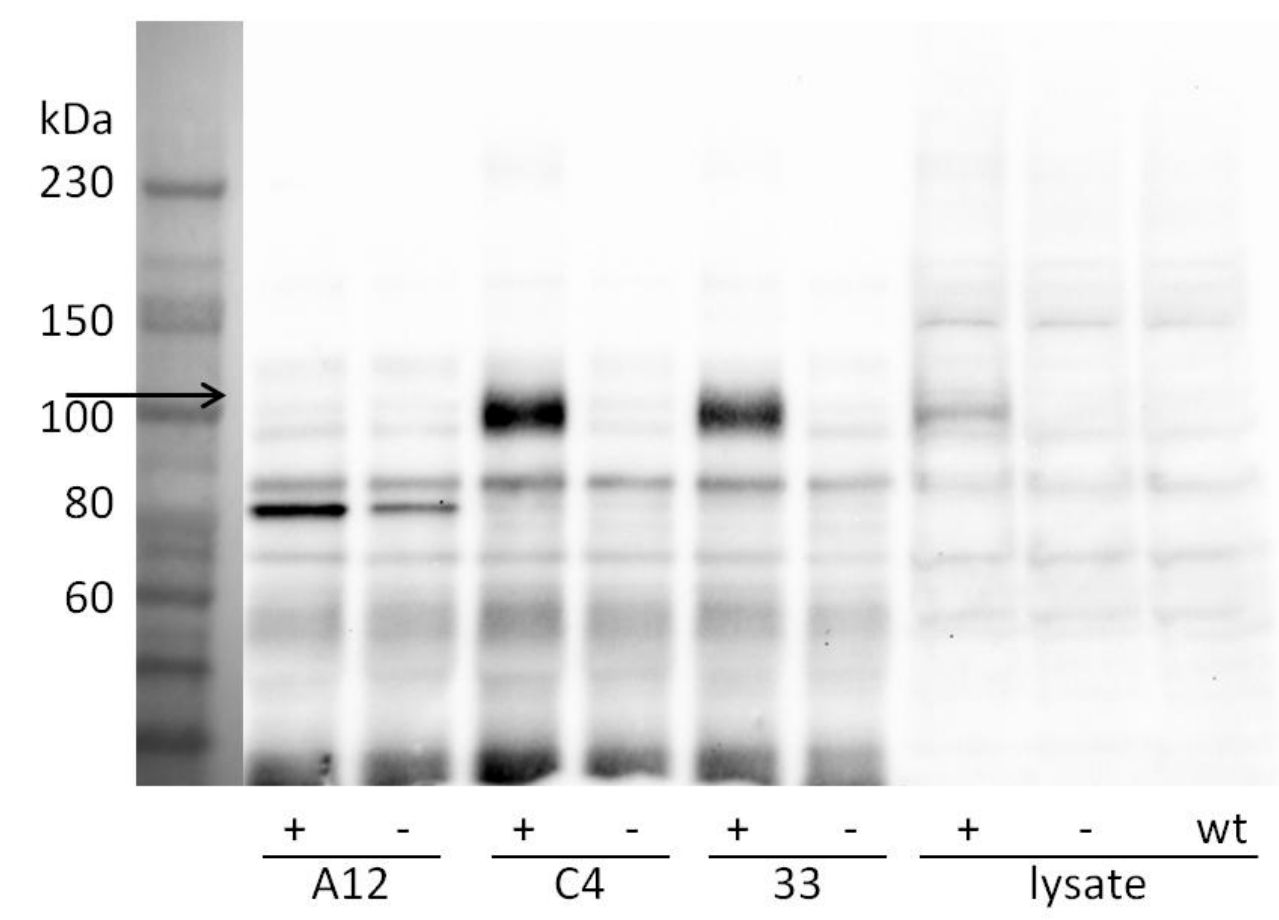

Figure 15: Detection of Kv10.1 by immunoprecipitation

Representative Western blot of immunoprecipitated Kv10.1 is shown. Anti-Kv10.1 nanobodies A12 and C4 as well as monoclonal anti-Kv10.1 antibody 33 were incubated with either pTracer-Kv10.1 (+) or pTracer- $\varnothing(-)$ transfected HEK cell lysates. Nanobodies were captured with an immobilized anti-myc antibody. Blotted precipitates were detected with polyclonal anti-Kv10.1 antibody 9391 resulting in clear bands at approx. $110 \mathrm{kDa}$ (indicated by arrow) in the pTracer-Kv10.1 transfected cell lysates but not in the pTracer- $\varnothing$ lysates with nanobody $C 4$ and antibody 33 as well as in the whole cell lysates (lysate). No band of 110 kDa could be detected with nanobody A12 or observed in wt HEK lysates.

\section{Enrichment of Kv10.1 for MALDI analysis}

Since a successful detection and enrichment of Kv10.1 was shown by IP for the nanobodies C4 and D9, the immunoprecipitates were further analyzed by matrix assisted laser desorption/ionization (MALDI) time of flight (TOF) mass spectrometry $(M S)^{3}$. A prerequisite for MS is a visible band in a Coomassie stained gel of the putative precipitated Kv10.1. To achieve this, the experimental approach had to be scaled up. As a first attempt, gels with the separated precipitate of nanobody D9 were silver stained because of the higher sensitivity of this assay compared to Coomassie stain. Using $1 \mathrm{mg}$ of pTracer-Kv10.1 and pTracer- $\varnothing$ transfected HEK whole cell lysate as well as wt HEK lysate an enrichment of a protein with a molecular weight of approximately $110 \mathrm{kDa}$ could be achieved by precipitation with $7.5 \mu \mathrm{g} / 0.5 \mathrm{nmol}$ nanobody D9,

\footnotetext{
${ }^{3}$ Mass spectrometry was performed by Thomas Liepold and PD Dr. Olaf Jahn.
} 
representing most likely Kv10.1 (Figure 16). This distinct band was not visible in the IP of the pTracer- $\varnothing$ transfected HEK cell lysate or wt cell lysate.

For MS analysis, the silver staining approach was adapted to the Coomassie staining approach because the silver ions and the fixative in the staining procedure interfere with the ionization during MS. Figure 17 shows the Coomassie stained gel after precipitation of putative Kv10.1 with nanobody D9. The bands in the range between 100 and $135 \mathrm{kDa}$ within the lane loaded with D9 precipitated pTracer-Kv10.1 transfected HEK cell lysates appeared a bit more intense compared to the HEK wild type cell lysates. Two spots out of each of the four bands were picked and loaded on a MALDI target for MS analysis. The detection of the precipitated Kv10.1 was performed by peptide mass fingerprinting, a MS technique using MALDI-TOF. This technique required four major steps. First, the protein was cleaved by tryptic digestion and loaded on a MALDI target. In a second step, the immobilized protein became ablated and desorbed by the laser and ionized by the hot plume of the ablated gases. The ions were accelerated in an electrical field and analyzed by the time of flight mass spectrometer in the third step. Finally, the masses of the detected ions were checked against a protein database. This validation failed because the amount of detected ions was not sufficient to generate a hit on the database. The next approach has to be scaled up to enrich more Kv10.1, the protein of interest. Analysis of the ionized fragments resulted in no hit assuming that the amount of ionized fragments was too low for MALDI analysis. 

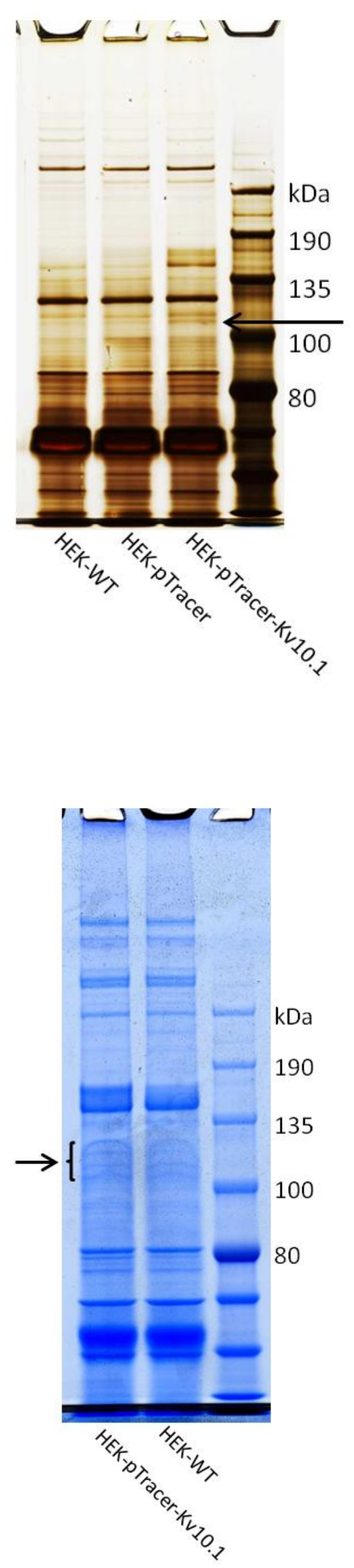

Figure 16: Silver stained enrichment of Kv10.1 by the use of nanobody D9

Anti-Kv10.1 nanobody D9 was incubated with either pTracerKv10.1 or pTracer- $\varnothing$ transfected HEK cell lysates or HEK-WT cell lysate. Nanobody D9 was captured with an immobilized anti-myc antibody. Precipitates were separated by SDS-PAGE and silver stained resulting in a clear band at approx. $110 \mathrm{kDa}$ (indicated by arrow) in the pTracer-Kv10.1 transfected lysate but not in the pTracer- $\varnothing$ transfected lysate or in HEK-WT cell lysate.

\section{Figure 17: Coomassie stain of precipitated Kv10.1}

Anti-Kv10.1 nanobody D9 was incubated with pTracer-Kv10.1 or pTracer- $\varnothing$ transfected HEK cell lysates. Nanobody D9 was captured with an immobilized anti-myc antibody. Immunoprecipitates were separated by SDS-PAGE and analyzed by Coomassie staining. A quartet of faint bands were observed in the range between 100 and $135 \mathrm{kDa}$ (indicated by bracket and arrow) in the pTracer-Kv10.1 transfected HEK cell lysate (left) compared to three faint bands in the HEK-WT lysate (right). 
Detection of the native Kv10.1 ion channel by flow cytometry

Flow cytometry experiments were performed to validate the detection of the native potassium channel Kv10.1 expressed on the surface on cells with anti-Kv10.1 nanobodies C4 and D9. As negative control, pTracer- $\varnothing$ transfected HEK cells were used. The pTracer plasmid expresses a cycle 3 green fluorescent protein (GFP) under control of a cytomegalovirus (CMV) promotor and Kv10.1 as protein of interest under control of a Simian vacuolating virus 40 (SV40) promotor. Thus, flow cytometry data can be gated retrospectively using the acquired GFP fluorescence, however the expression of GFP indicates the successful transfection but not the expression of the protein. Nanobodies C4 and D9 were revealed with a consecutive incubation with anti-myc and anti-mouse PE antibodies. At least 20,000 cells were counted for each experiment and debris was excluded. The mean fluorescence intensities (MFI) of all cells were assessed (Figure $18 \mathrm{~A}$ and B). Only a minimal increase of approximately 30 and $100 \mathrm{MFI}$ for nanobody D9 and C4, respectively, compared to baseline (second and third antibody only) could be determined. An increase in the range of orders of magnitude would indicate a high expression of Kv10.1. Assuming a poor expression of a functional Kv10.1 potassium channel on the surface of the pTracer-Kv10.1 transfected HEK cells, flow cytometry data were gated by wtGFP signals. Among the initially at least 20,000 gated cells only 4 - 30 cells per sample were positive for GFP and PE i.e. nanobody derived signal (Figure $18 \mathrm{C}$ and D). The MFI of the nanobodies doubled compared to baseline, but this result is statistically irrelevant due to the low number of cells (Figure $18 \mathrm{E}$ and $\mathrm{F}$ ), indicating a poor transfection efficiency. Therefore, it remains unclear if the nanobodies C4 and D9 detect the native ion channel Kv10.1 on cells. The experiment has to be repeated with better Kv10.1-transfected cells and more suitable reporter systems like the use of plasmids containing an internal ribosomal entry site entry site (IRES). 

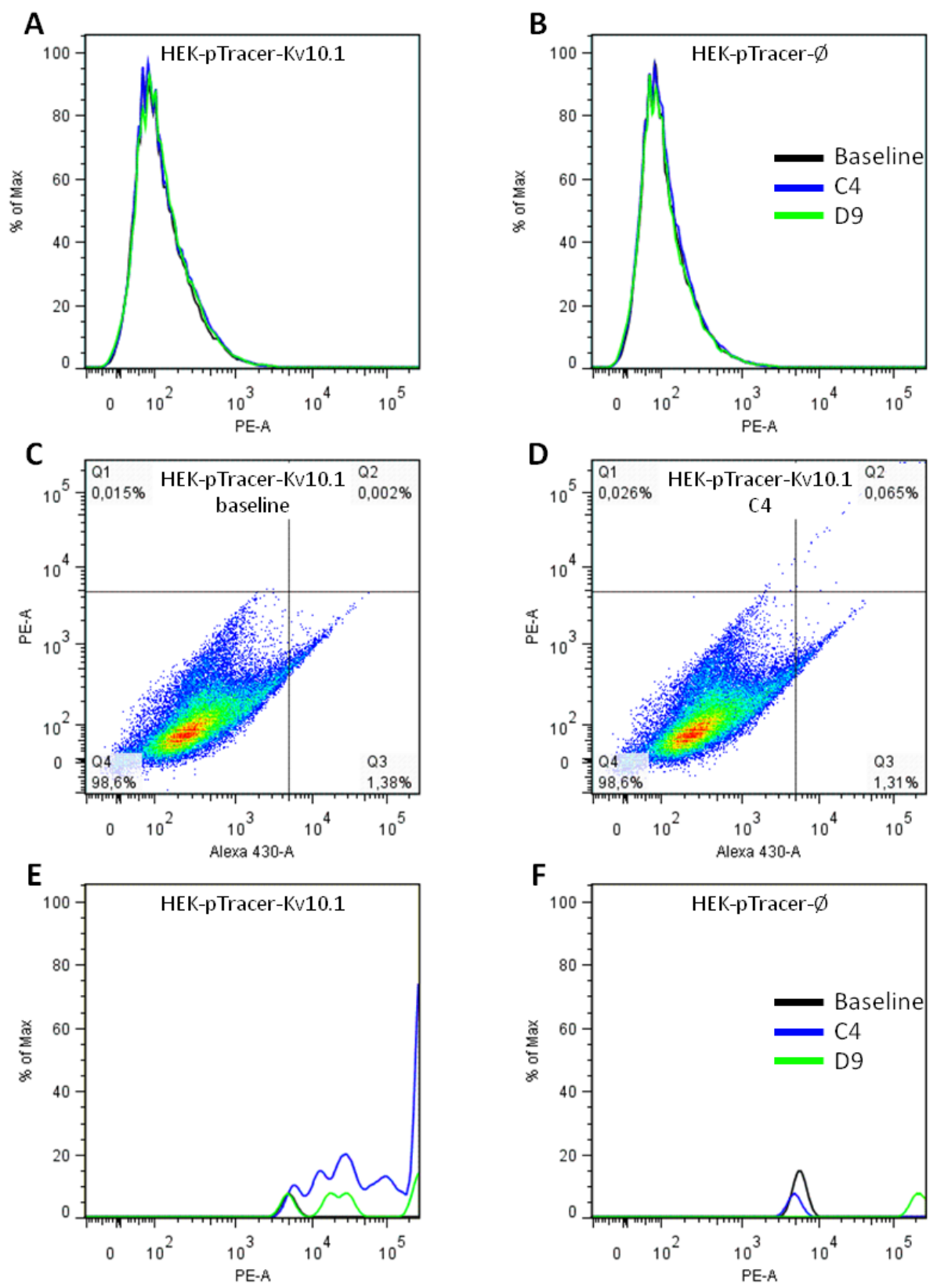

Figure 18: Flow cytometry of Kv10.1 transfected cells with nanobodies C4 and D9

Anti-Kv10.1 nanobodies C4 and D9 were incubated with (A) pTracer-Kv10.1 or (B) pTracer- $\varnothing$ transfected HEK cells. Nanobodies were revealed by consecutive incubation with an anti-myc and an anti-mouse PE antibody. Compared to baseline (black line), i.e. incubation with second and third antibody only, a minimal increase of approximately $100 \mathrm{MFI}$ could be determined for C4 in (A) pTracer-Kv10.1 transfected cells, but not in the (B) empty vector control. Due to the absence of pronounced signals after incubation with C4 and D9, the recorded cells were gated retrospectively for GFP since the pTracer plasmid expressed a cycle 3 GFP as reporter gene. The relation between GFP and PE fluorescence was displayed as scatter plot for (C) pTracer-Kv10.1 transfected HEK cells at (baseline) and (D) incubated with C4. Among initially 20,000 gated cells, only 4 to 30 were positive for GFP and PE. The MFI of the nanobodies in (E) pTracer-Kv10.1 transfected HEK cells doubled compared to baseline and (F) pTracer- $\varnothing$ transfected HEK cells, however this result is statistically irrelevant due to the low number of cells. 
The anti-Kv10.1 nanobodies were generated for the detection of Kv10.1 expressing tumors by in vivo SPECT. So far, the anti-Kv10.1 nanobodies C4 and D9 were identified as most promising clones out of 10 lead candidates. Nanobodies C4 and D9 were proven to specifically detect the native Kv10.1 on cells by IP. However, MALDI-TOF analysis to identify Kv10.1 still remains to be done. Furthermore, affinities of $1.7 \mu \mathrm{M}$ and $77 \mathrm{nM}$ towards the fusion protein $\mathrm{H} 1 \mathrm{X}$ were determined for nanobodies $\mathrm{C} 4$ and D9, respectively. For in vivo tumor visualization, a specific binding of the nanobodies C4 and D9 to the native ion channel on the surface of cells and/or in tissue slices remains to be elucidated.

Prior to in vivo application of anti-Kv10.1 nanobodies, the SPECT system had to be setup first. For validation of the SPECT system and for the development of the imaging procedure, involving radiolabeling, time of injection and time of the scan, duration of the scan, etc. well established tumor mouse models expressing EGFR were used. To achieve this, the clinically approved anti-EGFR antibody Cetuximab was applied first for tumor visualization as proof of principle followed by tumor visualization using an antiEGFR nanobody.

\subsection{Establishment of the SPECT infrastructure}

The work and transportation of radioactive substances is strictly regulated in Germany and rigorously controlled by local authorities. Hence all steps of the planned work were approved (Tgb.-Nr.: Ra 22/12-Gs/Wi) by local authorities prior start. Since no infrastructure for radiation protected mouse housing was on-site, a normal room was adapted and a self-made lead-shielded scantainer for mouse housing was installed.

The multi pinhole SPECT system was not used with technetium-99m before and had to be calibrated for the use of this radionuclide. For a quantification of the acquired images, the detected photons on the detector had to be related to the actual counts of

the sample. Calibration factors of $8.8 \times 10^{-4}$ and $8.3 \times 10^{-4}$ were determined for apertures 3 and 5, respectively.

The detectors of the clinical SPECT were tested every day for contamination and once a week for homogeneity. To assess the image quality and resolution of the multi- 
pinhole collimators and the reconstruction algorithm implemented in the HiSPECT software, scans with a Jaszczak phantom were carried out. The Jaszczak phantom contains rods with diameters of 1 to $2 \mathrm{~mm}$ and was filled with $2 \mathrm{ml}$ (approx. $6 \mathrm{GBq}$ ) ${ }^{99 \mathrm{~m}}$ Tc-pertechnetate and scanned with standard settings $\left(360^{\circ}\right.$ in 10 projections, $300 \mathrm{sec}$ acquisition per frame, $\mathbf{5 0 . 6}$ min total scan time) in SPECT using both apertures 3 and 5 with a FOV of $33 \times 35 \mathrm{~mm}$ and $50 \times 60 \mathrm{~mm}$, respectively. Figure 19 depicts the scan of the Jaszczak phantom with aperture 3 and 5 showing the different resolutions of the collimators. Single dots with a size of $1.2 \mathrm{~mm}$ and $2.0 \mathrm{~mm}$ in diameter were discriminated with aperture 3 and 5 , respectively.

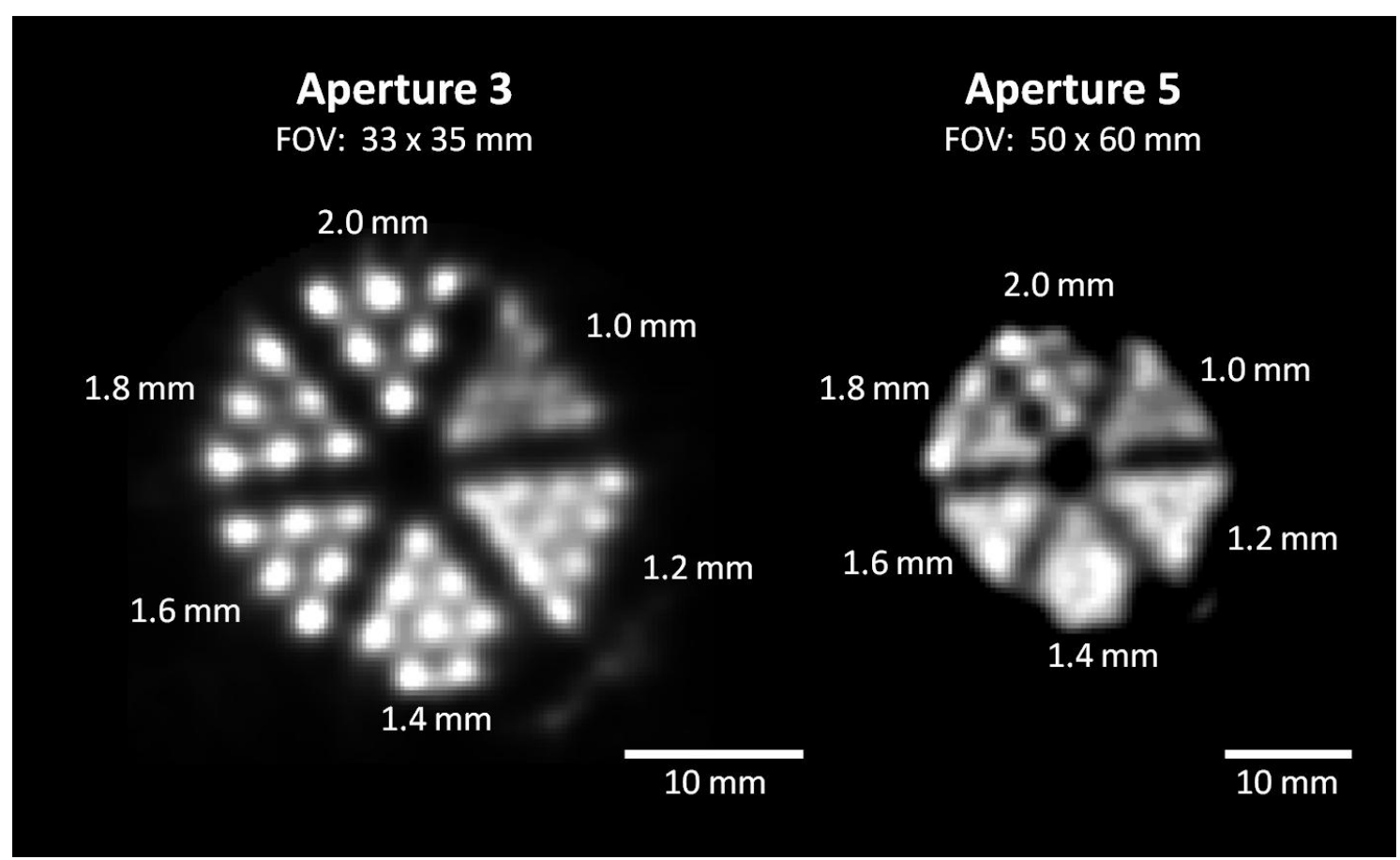

Figure 19: Jaszczak-Phantom scans using different collimators

The resolution of the multi pinhole SPECT system was determined by scans with a Jaszczak phantom. The phantom was filled with $2 \mathrm{ml}$ (approx. $6 \mathrm{GBq}$ ) ${ }^{99 \mathrm{~m}} \mathrm{Tc}-\mathrm{NaTCO}_{4}$ and scanned with standard settings $\left(360^{\circ}\right.$ in 10 projections, $300 \mathrm{sec}$ acquisition per frame, $50.6 \mathrm{~min}$ total scan time) in SPECT using both apertures 3 and 5 with a FOV of $33 \times 35 \mathrm{~mm}$ and $50 \times 60 \mathrm{~mm}$, respectively. Single dots with a size of $1.2 \mathrm{~mm}$ and $2.0 \mathrm{~mm}$ in diameter were discriminated with aperture 3 and 5 , respectively. 


\subsection{Validation of EGFR expressing tumor models for SPECT imaging}

Antibodies and nanobodies targeting the tumor marker EGFR, which is expressed on the surface of a variety of tumor cells, were used for setup of the SPECT system, for validation of the radiolabeling procedure and for proof of principal of tumor visualization of EGFR expressing tumors. Three well characterized cell lines with different published EGFR densities of $1.33 \times 10^{5} \mathrm{EGF}$ receptors/cell and $1.28 \times 10^{6} \mathrm{EGF}$ receptors/cells for the human mammary carcinoma cell lines MDA-MB-231 and MDAMB-468 [94], respectively, and $2 \times 10^{6}$ EGF receptors/cell for the human epidermoid cancer cell line A431 [95] were used for tumor xenograft mouse models.

\section{Validation of EGFR expression by Western blotting}

First, protein expression of EGFR was investigated in human tumor cells by Western blotting analysis (Figure 20). A low EGFR expression level for MDA-MB-231 and high EGFR expression levels for MDA-MB-468 and A431 cells were determined.

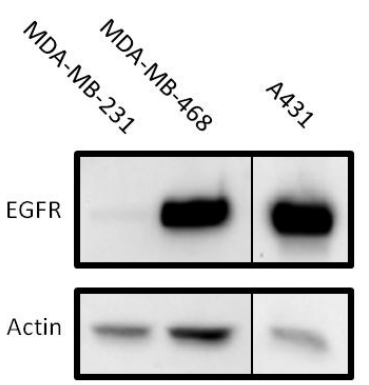

\footnotetext{
Figure 20: Validation of EGFR expression by Western blotting

Western blot analysis of EGFR expression levels of cell lysates of human mammary carcinoma MDA-MB-231 and MDA-MB-468 cells as well as of human epidermoid carcinoma A431 cells are shown. High levels of EGFR (band at approx. $170 \mathrm{kDa}$ ) expression were found in MDA-MB468 and $A 431$ cells compared to low EGFR expression in MDA-MB-231 cells. Actin (band at approx. $40 \mathrm{kDa}$ ) was used as loading control to monitor differences in the amount of loaded protein.
}

\section{Detection of the native EGFR by flow cytometry}

EGFR expression on the surface of the used cell lines was validated by flow cytometry using the clinically approved anti-EGFR antibody Cetuximab. This experiment was performed to validate the accessible EGFR expressed on tumor cells and to assess the suitability of Cetuximab as imaging probe. Therefore, the human tumor cells were incubated with Cetuximab (blue line) and revealed with Protein $\mathrm{G}$ coupled to AlexaFluor 488 (Figure 21). Unspecific binding was ruled out by incubation of the human tumor cells with a human IgG1 isotype antibody (gray line) that remained at 
baseline levels (black line) (Figure 21A-C). The ratios of the obtained Cetuximab MFI and baseline MFI (Figure 21D) confirmed low EGFR expression for MDA-MB-231 and high expression for MDA-MB-468 and A431 cells. The ability to bind EGFR on the surface of the cells indicates Cetuximab as a possibly useful imaging probe for in vivo SPECT targeting EGFR.

A

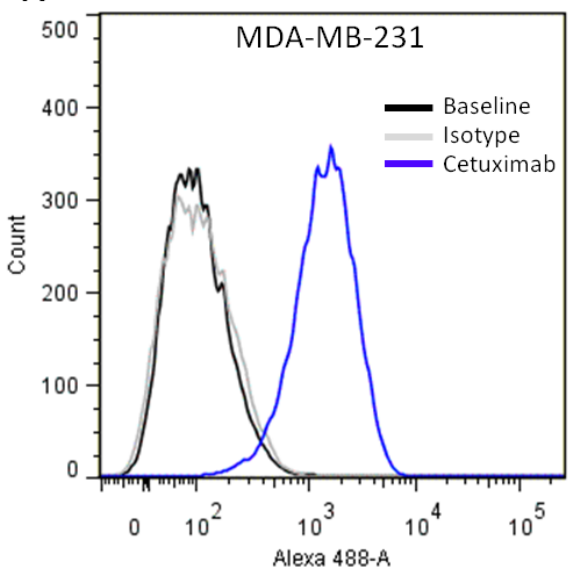

C

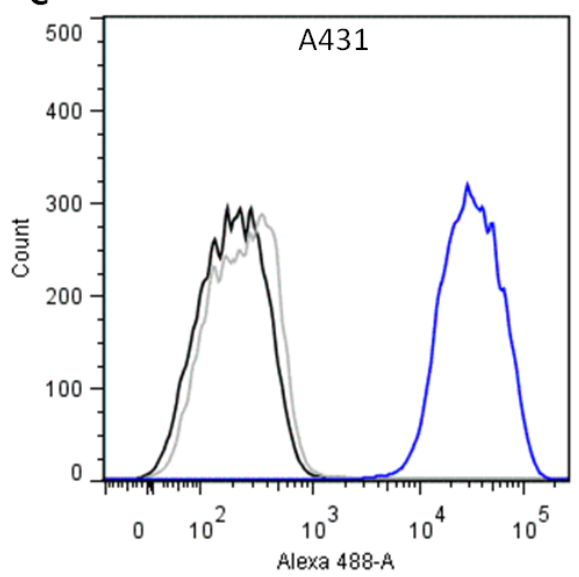

B

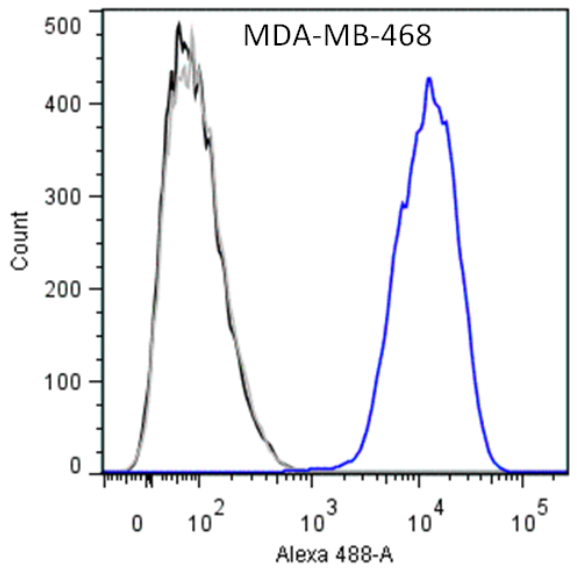

D

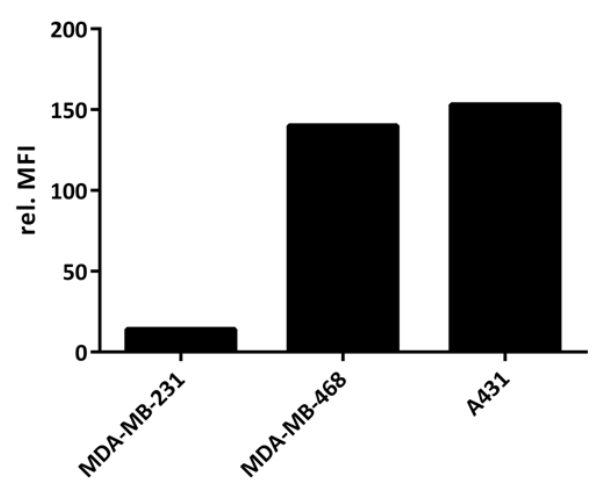

Figure 21: Validation of EGFR expression by flow cytometry

The expression of EGFR was assessed for human mammary carcinoma cells (A) MDA-MB-231 and (B) MDA-MB-468 as well as (C) epidermoid carcinoma cells A431 with Cetuximab (blue line) and compared to the $\operatorname{lgG}_{1}$ isotype control (grey line) as well as baseline (black line) by flow cytometry. High EGFR expression levels were proven for MDA-MB-468 and A431 and a low EGFR expression level for MDAMB-231 cells. All antibodies were revealed by incubation with Protein G coupled to AlexaFluor 488. MFI of isotype antibody matched the baseline MFI values (i.e. incubation with Protein G-AF488 only), ruling out any unspecific binding to the tumor cells. (D) EGFR expression was displayed as ratio of Cetuximab MFI to baseline MFI values. $2 \times 10^{4}$ cells per sample were recorded and the MFIs were displayed as histograms. 
Validation of EGFR expression on tumor tissue slices of xenografts by IHC

Following the determination of EGFR expression levels in vitro, the EGFR expression was assessed ex vivo on tumor tissue slices by IHC. Mammary carcinoma cells were injected orthotopically in the fat pad of the right abdominal mammary gland whereas the epidermoid tumor cell line was implanted subcutaneously on the right flank. The tumors were allowed to grow for 4 to 8 weeks to a diameter of 3 to $5 \mathrm{~mm}$ before they were dissected and analyzed by IHC for EGFR expression ${ }^{4}$. In accordance to the previously acquired in vitro data on cells, staining of tumor tissue with a mouse monoclonal anti-EGFR antibody clone SP9 revealed a minor, light brownish stain for a low EGFR expression in MDA-MB-231 and a positive, intense brownish stain for a high EGFR expression in both, MDA-MB-468 and A431 tumors (Figure 22). Interestingly, in MDA-MB-231 and MDA-MB-468 tumors, EGFR expression was found to be high on the border between tumor and surrounding tissue and to be distinctly decreased with distance to the border to the inner side of the tumor. MDA-MB-231 tumors had a very narrow border of EGFR expressing cells surrounding necrotic tissue whereas A431 tumors were highly EGFR positive throughout the entire tumor.

\footnotetext{
${ }^{4}$ EGFR staining on tumor tissue sections was performed by Bettina Jeep and Sabine Wolfgramm.
} 


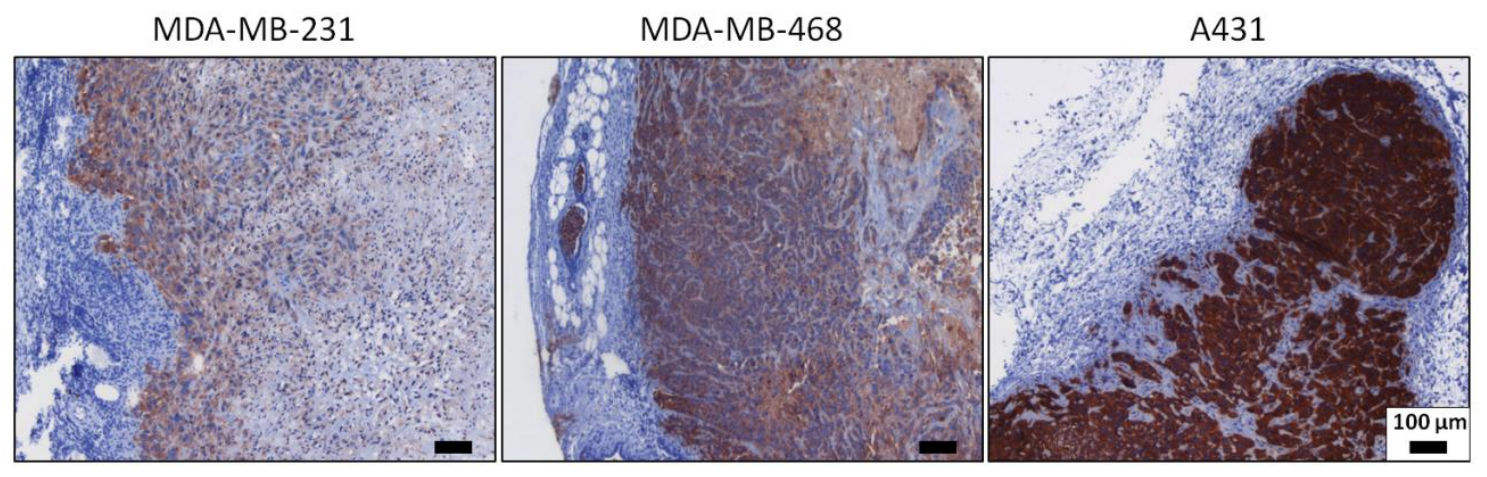

Figure 22: Validation of EGFR expression levels in tumors by IHC

Paraffin tissue sections $(2 \mu \mathrm{m})$ of orthotopic MDA-MB-231 (tumor volume: $200 \mathrm{~mm}^{3}$ ) and MDA-MB-468 $\left(45 \mathrm{~mm}^{3}\right)$ as well as subcutaneous A431 $\left(28 \mathrm{~mm}^{3}\right)$ tumors were analyzed by IHC for EGFR expression. Staining of tumor tissue with a mouse monoclonal anti-EGFR antibody clone SP9 revealed minor staining of tumor cells for a low EGFR expression in MDA-MB-231 (left) and a positive, intense brown stain for a high EGFR expression in MDA-MB-468 (middle) and A431 tumors (right). In MDA-MB-231 and MDA-MB468 tumors, EGFR expression was high on the border between tumor and surrounding tissue and distinctly decreased with distance to the border to the inner side of the tumor. MDA-MB-231 tumors were characterized by a very narrow border of EGFR expressing cells surrounding necrotic tissue. A431 tumors were highly EGFR positive throughout the entire tumor. EGFR was stained with DAB and nuclei were counterstained with hematoxylin. Brightfield microscopy was performed with 10x magnification. The scale bar represents $100 \mu \mathrm{m}$.

The expression of EGFR on MDA-MB-231, MDA-MB-468 and A431 cell lines as well as on the tumors was determined by Western blotting, flow cytometry and IHC. In all experiments, high EGFR levels were determined for MDA-MB-468 and A431 cells in contrast to low EGFR expression levels observed in MDA-MB-231 cells. Especially the verification of the specific binding of Cetuximab to EGFR on tumor cells indicates that targeting of EGFR in combination with Cetuximab might be a suitable approach for in vivo tumor visualization by SPECT.

\subsection{Radiolabeling}

\section{Derivatization of full IgGs with HyNic}

Full IgG antibodies such as Cetuximab and the human IgG1 isotype control were modified with the bifunctional chelator $\mathrm{HyNic}$ to enable radiolabeling with reduced ${ }^{99 \mathrm{~m}} \mathrm{Tc}(\mathrm{II})$. Since HyNic was conjugated to the terminal $\varepsilon$-amino groups of lysines, the impact of the modification on the binding properties of Cetuximab to EGFR was determined by flow cytometry. MDA-MB-468 tumor cells were incubated with 
Cetuximab and Cetuximab-HyNic concentrated from $10 \mathrm{nM}$ to $3.12 \mathrm{pM}$, detected by incubation with Protein G-AF488 and subsequent measurement by flow cytometry. For analysis, the measured MFIs were fitted to a one side total binding model. Equilibrium binding constants $K_{d}$ of $417 \mathrm{pM}$ and $442 \mathrm{pM}$ were determined for Cetuximab and Cetuximab-HyNic, respectively. Interestingly, lower MFIs were detected after incubation of the modified Cetuximab-HyNic at a saturation level at $2.5 \mathrm{nM}$ indicating either fewer antibodies bound to the EGFR on the cell surface, or fewer molecules Protein $\mathrm{G}$ bound to the conjugated Cetuximab-HyNic compared to the unlabeled Cetuximab. The latter is more likely since the modification with HyNic might interfere with the binding of Protein $\mathrm{G}$ and $\mathrm{K}_{\mathrm{d}}$ remained unaltered after modification.

A

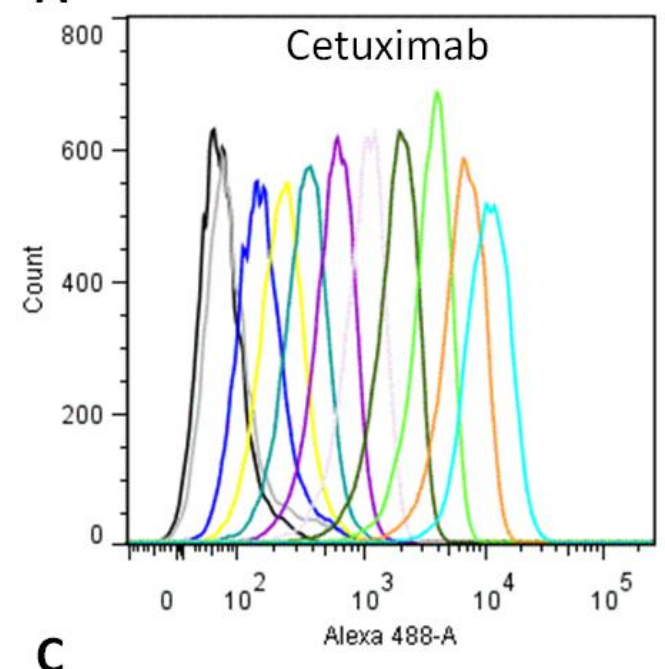

C

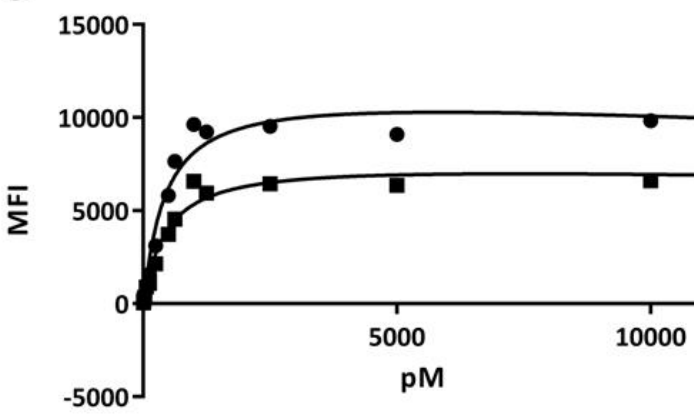

B
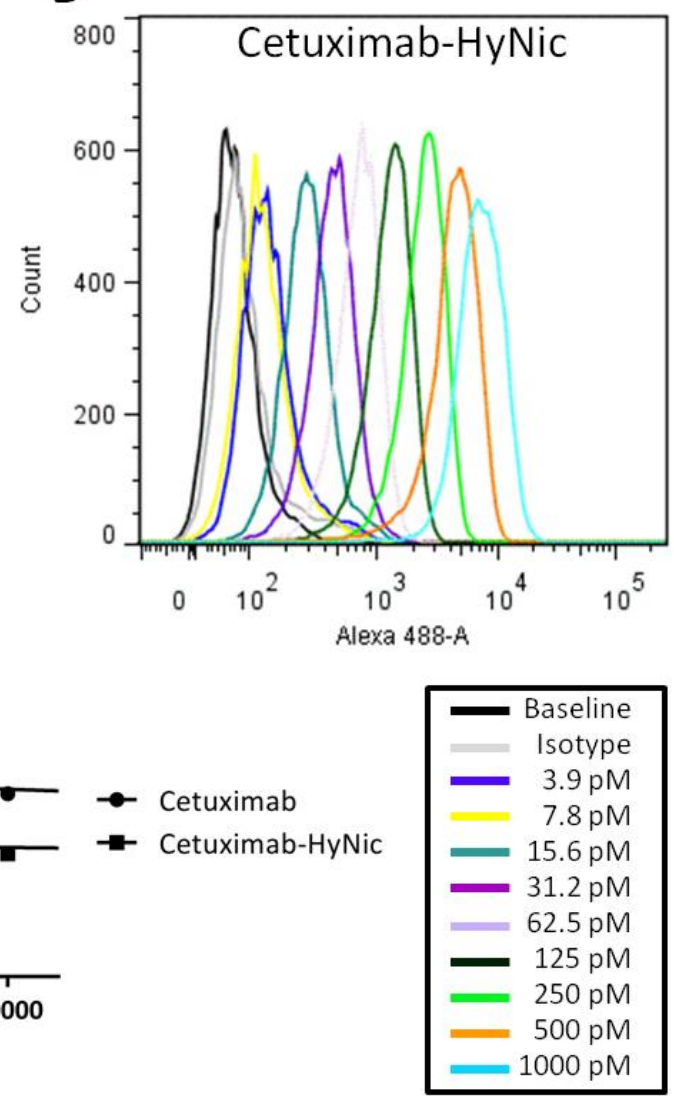

Figure 23: Modification of Cetuximab did not alter the $K_{d}$

Equilibrium binding constants $K_{d}$ of $(A)$ Cetuximab and of (B) Cetuximab-HyNic were determined in a titration assay on MDA-MB-468 cells by flow cytometry. For analysis, the measured MFIs were (C) fitted to a one side total binding model. Equilibrium binding constants $K_{d}$ of $417 \mathrm{pM}$ and $442 \mathrm{pM}$ were determined for Cetuximab and Cetuximab-HyNic, respectively. MDA-MB-468 tumor cells were incubated with Cetuximab and Cetuximab-HyNic in concentrations from $10 \mathrm{nM}$ to $3.12 \mathrm{pM}$. Antibodies were revealed by subsequent incubation with Protein G-AF488. Lower MFIs were detected after incubation of the modified Cetuximab-HyNic at a saturation level of $2.5 \mathrm{nM}$. $2 \times 10^{4}$ cells per sample were recorded and MFIs are displayed as histograms. For reasons of clarity, only concentrations from baseline (i.e. incubation ProteinG-AF488 only) to $1 \mathrm{nM}$ are displayed. 


\section{Radiolabeling of HyNic-modified antibodies}

Labeling of HyNic modified antibodies, Cetuximab and isotype control, with ${ }^{99 \mathrm{~m}} \mathrm{Tc}$ sodium pertechnetate $\left({ }^{99 \mathrm{~m}} \mathrm{Tc}-\mathrm{NaTcO}_{4}\right)$ and tricine as coligand resulted in a specific activity of $1700 \mathrm{MBq} / \mathrm{nmol} \pm 105(\mathrm{~N}=25)$ with a yield of $44.1 \pm 10.1 \%$ and a purity of 97.3\% for ${ }^{99 \mathrm{~m}}$ Tc-Cetuximab (Table 18 ).

\section{Radiolabeling of anti-EGFR nanobodies via 6xHis-tag}

The anti-EGFR D10 and the irrelevant control nanobody F5 were labeled sitespecifically with $\left[{ }^{99 m} \mathrm{Tc}(\mathrm{CO})_{3}\right]^{+}$via the $6 x$ His tags of the nanobodies. A specific activity of $183 \mathrm{MBq} / \mathrm{nmol} \pm 35$ and $182 \mathrm{MBq} / \mathrm{nmol} \pm 51$ and a radiochemical yield of $48.8 \% \pm 7.0$ and $58.2 \% \pm 6.7$ were obtained for the anti-EGFR nanobody ${ }^{99 m}$ Tc-D10 $(N=12)$ and for the control nanobody ${ }^{99 \mathrm{~m}} \mathrm{Tc}-\mathrm{F} 5(\mathrm{~N}=10)$, respectively. After purification, radiochemical purities, i.e. the amount of protein-bound activity, of $97.7 \% \pm 1.2\left({ }^{99 \mathrm{~m}} \mathrm{Tc}-\mathrm{D} 10\right)$ and $98.2 \% \pm 1.1\left({ }^{99 \mathrm{~m}} \mathrm{Tc}-\mathrm{F} 5\right)$ were determined by ITLC (Table 18).

Stability of each radiolabeled antibody ${ }^{99 \mathrm{~m}} \mathrm{Tc}$-Cetuximab and nanobodies ${ }^{99 \mathrm{~m}} \mathrm{Tc}-\mathrm{D} 10$ and ${ }^{99 \mathrm{~m}}$ Tc-F5 was tested by instant thin layer chromatography (ITLC) after incubation in mouse serum at $37^{\circ} \mathrm{C}$ for different durations. More than $90 \%$ and $95 \%$ of the activity remained bound to the nanobodies and to the full IgG antibody after $4 \mathrm{~h}$ incubation, respectively.

Table 18: Parameters of radiolabeling

\begin{tabular}{lcc}
\hline & nanobody ( ${ }^{99 m}$ Tc-D10) & IgG $_{1}$ antibody ( ${ }^{99 m}$ Tc-Cetuximab) \\
\hline Labeling method & {$\left[{ }^{99 \mathrm{~m}} \mathrm{Tc}(\mathrm{CO})_{3}\right]^{+}$on $6 x$ His tag } & $\begin{array}{c}\text { modification with HyNic / tricine as } \\
\text { coligand }\end{array}$ \\
Site-specific & Yes & No \\
Specific activity & $183 \pm 35 \mathrm{MBq} / \mathrm{nmol}$ & $1700 \pm 105 \mathrm{MBq} / \mathrm{nmol}$ \\
Radiochemical yield & $48.8 \% \pm 7.0$ & $44.1 \% \pm 10.1$ \\
Radiochemical purity & $97.7 \% \pm 1.2$ & $97.3 \% \pm 0.4$ \\
\hline
\end{tabular}

Binding properties of the unlabeled Cetuximab to EGFR were analyzed on A431 tumor cells expressing high EGFR levels using flow cytometry and were compared to the radiolabeled ${ }^{99 \mathrm{~m}} \mathrm{Tc}$-Cetuximab after decay of the radioactivity. Figure 24 depicts a slight 
decrease of the MFIs of Cetuximab-HyNic (green line) and ${ }^{99 \mathrm{~m}}$ Tc-Cetuximab (red line) compared to results obtained by Cetuximab alone (blue line). All derivatives show a distinct increase in MFI in the range of two orders of magnitude compared to baseline (Protein G-AF488 only, black line) proving the binding of Cetuximab to EGFR expressed on A431 cells. The slight decrease observed for the MFIs of the modified Cetuximab derivatives can be explained by the modification of the Fc portion of the antibody with HyNic, that might hamper the binding of Protein $G$ to the Fc portion.

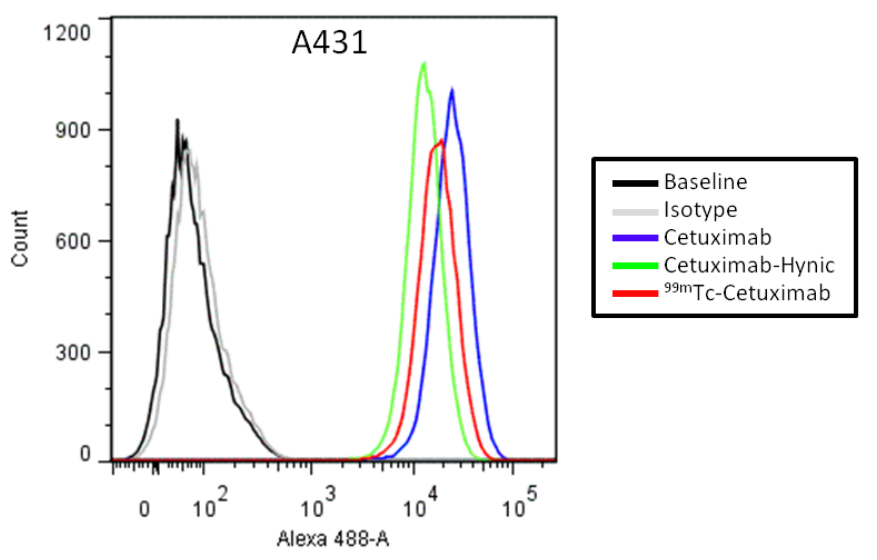

Figure 24: Radiolabeled Cetuximab binds to EGFR

Binding capacities of Cetuximab (blue line), Cetuximab-HyNic (green line) and radiolabeled ${ }^{99 \mathrm{~m}} \mathrm{Tc}$ Cetuximab (red line) to EGFR expressed on human mammary carcinoma cells MDA-MB-468 were investigated by flow cytometry and compared to the human $\operatorname{lgG}_{1}$ isotype control (grey line). All antibodies were revealed by incubation with Protein $G$ coupled to AlexaFluor 488. No pronounced alteration of the binding properties to EGFR were caused by the radiolabeling procedure. However, a slight decrease of both, MFIs of modified Cetuximab compared to unmodified Cetuximab was detected and might be due to an impaired binding of Protein $G$ to the Fc portion of the modified antibody. MFIs of IgG1 isotype matched the MFI values of baseline (i.e. incubation with Protein G-AF488 only), ruling out any unspecific binding of Cetuximab to the tumor cells. $2 \times 10^{4}$ cells per sample were recorded and the MFIs are displayed as histograms.

\section{Detection of EGFR with anti-EGFR nanobody D10 by flow cytometry}

In analogy to the analysis of the full $\lg _{1}$ Cetuximab, binding properties of the unlabeled nanobodies D10 and F5 to endogenously expressed EGFR on A431, MDAMB-231 and MDA-MB-468 tumor cells were compared to results of the radiolabeled nanobodies ${ }^{99 m} \mathrm{Tc}-\mathrm{D} 10$ and ${ }^{99 \mathrm{~m}} \mathrm{Tc}-\mathrm{F} 5$ using flow cytometry after decay of the radioactivity. In all three cell lines no differences in the MFIs between the radiolabeled ${ }^{99 \mathrm{~m}} \mathrm{Tc}-\mathrm{D} 10$ and ${ }^{99 \mathrm{~m}} \mathrm{Tc}-\mathrm{F} 5$ (red line) and unlabeled (blue line) nanobodies D10 and F5 
were measured. This demonstrates that the radiolabeling procedure caused no alteration of the binding properties of the nanobody to EGFR (Figure 25). As expected, irrelevant nanobodies F5 and ${ }^{99 m}$ Tc-F5 were negative on all cell lines (light blue and red lines) ruling out an unspecific binding of nanobodies to the investigated tumor cells. The MFI of the anti-EGFR nanobody D10 correlated well with the receptor density on the cell surface of A431 and MDA-MB-468 cells, both characterized by a high level of EGFR expression (Figure 25B+C). No detectable EGFR expression could be detected on MDA-MB-231 cells (Figure 25A) representing the detection limit for the anti-EGFR nanobody D10 since no increase of the MFI compared to baseline could be detected under these conditions.
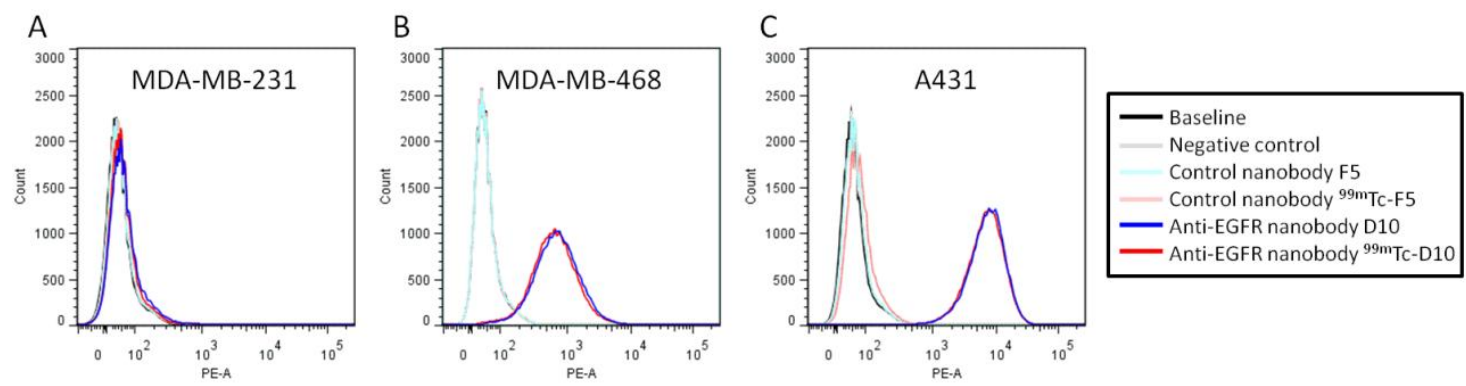

Figure 25: Assessment of binding capacities of radiolabeled nanobodies

Binding capacities of anti-EGFR nanobody D10 (blue line) and radiolabeled ${ }^{99 m}$ Tc-D10 (red line) to EGFR expressed on (A) mammary carcinoma cells MDA-MB-231 and (B) MDA-MB-468 as well as (C) epidermoid carcinoma cells $A 431$ were investigated by flow cytometry and compared to the results obtained by the irrelevant control nanobody F5 (light blue line) and by the radiolabeled ${ }^{99 m}$ Tc-F5 (light red line). All nanobodies were revealed by consecutive incubation with an anti-myc antibody and goatanti-mouse antibody coupled to PE. No alteration of the binding properties to EGFR caused by the radiolabeling procedure were observed. MFIs of control nanobody F5 matched the MFI values of background and negative control (i.e. incubation with anti-myc antibody and goat-anti-mouse-PE only), ruling out any unspecific binding to the tumor cells. $5 \times 10^{4}$ cells per sample were recorded and the MFIs are displayed as histogram.

\subsection{SPECT scans}

\subsubsection{Initial SPECT scans}

Labeling of nanobodies and antibodies with generator-based ${ }^{99 \mathrm{~m}} \mathrm{Tc}$-sodium pertechnetate (oxidation state $+\mathrm{VII}$ ) always involves a reduction to remove oxygen atoms and permit the establishment of covalent bonds between the nitrogen atoms of 
the modified antibody or the histidine of the nanobodies with the Tc-core. The reduction with tin chloride to generate Tc (oxidation state + II) suitable for the labeling of HyNic-modified antibodies can lead to the formation of colloids. However, the addition of tricine as a coligand prevents the formation of colloids and stabilizes the Tc-atom on the nitrogen of the HyNic chelator. In order to rule out unspecific signals from unbound activity or aggregates during the labeling process, initial SPECT scans with all chemicals used for the radiolabeling, such as ${ }^{99 \mathrm{~m}} \mathrm{Tc}_{\mathrm{NaTcO}}$, Tc-colloids and tin (Sn)-reduced Tc with tricine as coligand, were carried out in healthy non-tumor bearing nude mice. Approximately $100 \mathrm{MBq}$ of the respective tracer were injected i.v. and SPECT scans were performed at different time points from $5 \mathrm{~min}$ to $24 \mathrm{~h}$ post injection (Figure 26). After the last scan at $24 \mathrm{~h}$ post injection the mice were sacrificed and the remaining activity in the organs was measured in a gamma counter.

Shortly, 5 min after administration of ${ }^{99 \mathrm{~m}} \mathrm{Tc}^{-N_{a T c O}}$ an uptake in the thyroid and the stomach could be observed. In the course of investigation, activity was detected in the bladder after $25 \mathrm{~min}$ and in the intestine with increasing signals from 90 min to $6 \mathrm{~h}$. After $24 \mathrm{~h}$ activities could be detected in the stomach and intestine (Figure 26). Ex vivo biodistribution analysis revealed that the activity determined in the intestine was derived from the chymen. Since the food was not supposed to be radioactive, the empty stomach and the stomach content were analyzed separately, detecting high activities in both, empty stomach and stomach content. This suggested an uptake in the stomach by the sodium/iodine symporter of ${ }^{99 \mathrm{~m}} \mathrm{Tc}-\mathrm{NaTcO}_{4}$ and a subsequent passage in the lumen and intestine (Figure 27). A similar distribution pattern, uptake via the stomach, was observed after the injection of tin reduced ${ }^{99 \mathrm{~m}} \mathrm{Tc}-\mathrm{NaTcO}_{4}$ with tricine as coligand that was produced analog to the labeling procedure of the HyNicmodified antibodies. A very different distribution pattern was achieved by the use of Tc-colloids prior to the addition of the coligand tricine that prevents the formation of Tc-colloids. The administration of Tc-colloids resulted in a high uptake in the liver and spleen of $45.2 \% \mathrm{ID} / \mathrm{g} \pm 6.7$ and $21 \% \mathrm{ID} / \mathrm{g} \pm 1.4$, respectively after $24 \mathrm{~h}$ post injection determined by ex vivo biodistribution analysis. After $24 \mathrm{~h}$ all tracers were completely removed from the blood pool resulting in remaining activities in the blood ranging from $0.01 \%$ ID/g to $0.05 \%$ ID/g (Figure 27 ). 
${ }^{99 \mathrm{~m}} \mathrm{Tc}-\mathrm{NaTcO}_{4}$

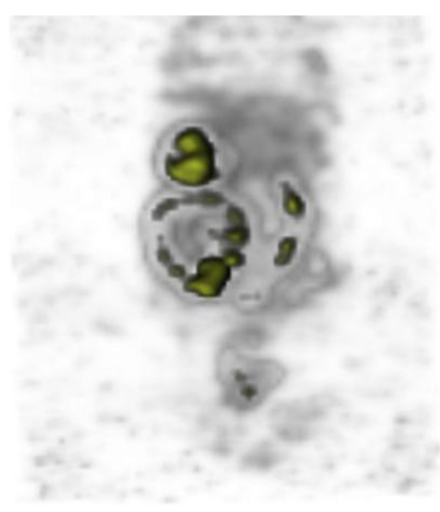

Sn reduced ${ }^{99 \mathrm{~m}} \mathrm{Tc}+$ Tricine

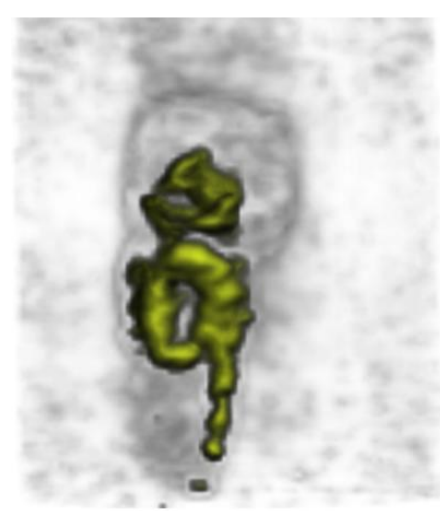

${ }^{99 \mathrm{~m} T c}$ colloids

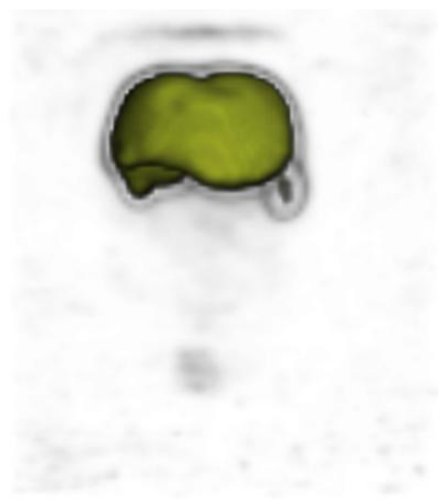

Figure 26: In vivo SPECT scans of ${ }^{99 \mathrm{~m}} \mathrm{Tc}$ tracers

Initial SPECT scans after injection of different chemicals used for the radiolabeling, ${ }^{99 \mathrm{~m}} \mathrm{Tc}^{-\mathrm{NaTcO}_{4}}$ (left), tin (Sn)-reduced Tc with tricine as coligand (middle) and Tc colloids (right) were carried out in healthy non-tumor bearing nude mice. Approximately $100 \mathrm{MBq}$ of the respective tracer were injected i.v. and SPECT scans were performed $24 \mathrm{~h}$ post injection. After application of ${ }^{99 \mathrm{~m}} \mathrm{Tc}-\mathrm{NaTcO}_{4}$ and $\mathrm{Sn}$ reduced Tc with tricine as coligand activities in the stomach and intestine could be detected, whereas the application of Tc colloids resulted in pronounced liver and spleen signals. Since data are presented as 3D maximum intensity projection, the signals in the different images are not comparable.
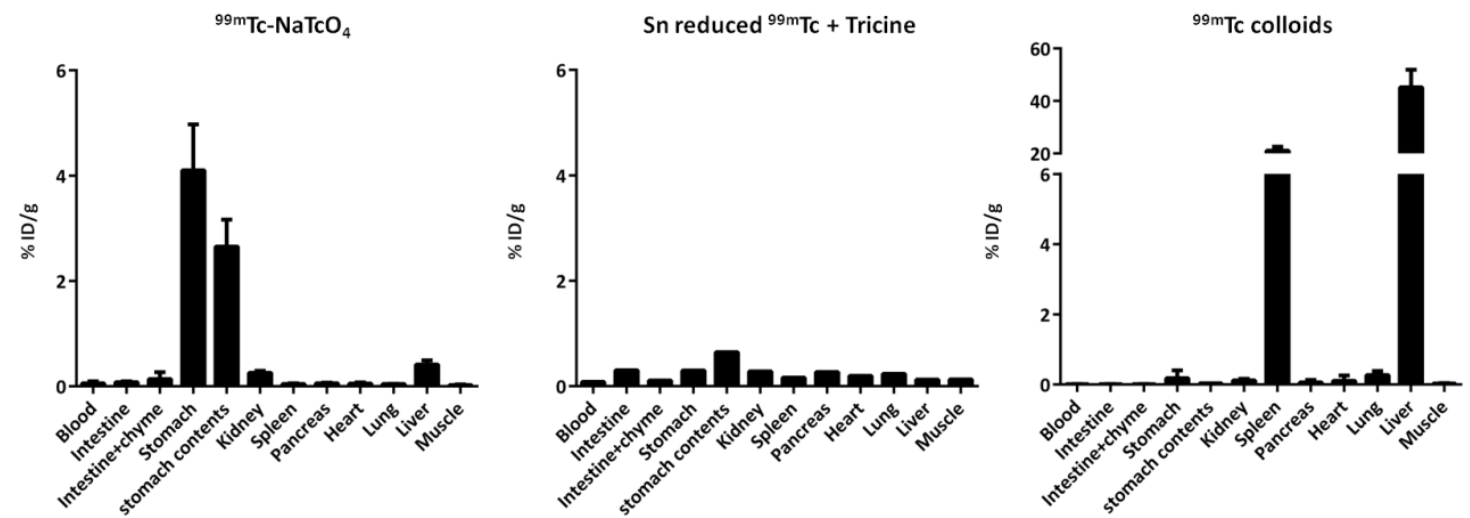

Figure 27: Ex vivo biodistribution analysis of ${ }^{99 \mathrm{~m}}$ Tc-tracers

The uptakes of the intermediates for Cetuximab labeling, ${ }^{99 \mathrm{~m}} \mathrm{Tc}-\mathrm{NaTcO}_{4}$, tin reduced ${ }^{99 \mathrm{~m}} \mathrm{Tc}$-technetium with coligand tricine and ${ }^{99 \mathrm{~m}} \mathrm{Tc}$ colloids to the organs were assessed by ex vivo biodistribution analysis $24 \mathrm{~h}$ post i.v. injection to non-tumor bearing nude mice. For ${ }^{99 \mathrm{~m}} \mathrm{Tc}^{-\mathrm{NaTcO}_{4}}(\mathrm{~N}=3$, left) high uptakes in the stomach $(4.1 \% \mathrm{ID} / \mathrm{g} \pm 0.9)$ and in the stomach content $(2.6 \% \mathrm{ID} / \mathrm{g} \pm 0.5)$ was observed. Except for liver $(0.4 \% \mathrm{ID} / \mathrm{g} \pm 0.1)$ and intestine with chyme $(0.1 \% \mathrm{ID} / \mathrm{g} \pm 0.1)$ the uptake of ${ }^{99 \mathrm{~m}} \mathrm{Tc}-\mathrm{NaTcO}_{4}$ to all other tissues was below $0.1 \% \mathrm{ID} / \mathrm{g}$. After injection of tin reduced ${ }^{99 \mathrm{~m}} \mathrm{Tc}$-technetium with coligand tricine in a mouse (middle) uptakes below $0.6 \% \mathrm{ID} / \mathrm{g}$ to all organs were determined. Application of ${ }^{99 \mathrm{~m}} \mathrm{Tc}$ colloids $(\mathrm{N}=3$, right) resulted in a high uptake in the liver and spleen of $45.2 \% \mathrm{ID} / \mathrm{g} \pm 6.7$ and $21 \% \mathrm{ID} / \mathrm{g} \pm 1.4$, respectively after $24 \mathrm{~h}$ post injection. After $24 \mathrm{~h}$ all tracers were completely removed from the blood pool resulting in determined activities of the blood with values ranging from $0.01 \% \mathrm{ID} / \mathrm{g}$ to $0.05 \% \mathrm{ID} / \mathrm{g}$. Data are shown as mean \pm SD in \% ID/g. 


\subsubsection{Tumor visualization with ${ }^{99 \mathrm{~m}} \mathrm{Tc}$-Cetuximab}

Blood clearance of ${ }^{99 m} T c$-Cetuximab

Since the labeling of Cetuximab with reduced ${ }^{99 m}$ Tc-pertechnetate was successful, ${ }^{99 m}$ Tc-Cetuximab was used for the visualization of primary MDA-MB-231 and MDA-MB468 mammary carcinomas that developed after orthotopic cell implantation. Before using the ${ }^{99 m}$ Tc-Cetuximab in SPECT, the clearance from blood pool was determined in a non-tumor bearing mouse to estimate the best time point for SPECT scans, i.e. when the remaining activity in the blood reached its plateau (Figure 28). A serum half-life of $180 \mathrm{~min}$ with a plateau of $6.1 \% \mathrm{ID} / \mathrm{g} \pm 0.89$ were identified for ${ }^{99 \mathrm{~m}} \mathrm{Tc}$-Cetuximab allowing imaging from approx. $16 \mathrm{~h}$ post injection. For organizational matters SPECT scans were performed with ${ }^{99 \mathrm{~m}} \mathrm{Tc}$-Cetuximab approx. $24 \mathrm{~h}$ post injection.

\section{${ }^{99 m}$ Tc-Cetuximab}

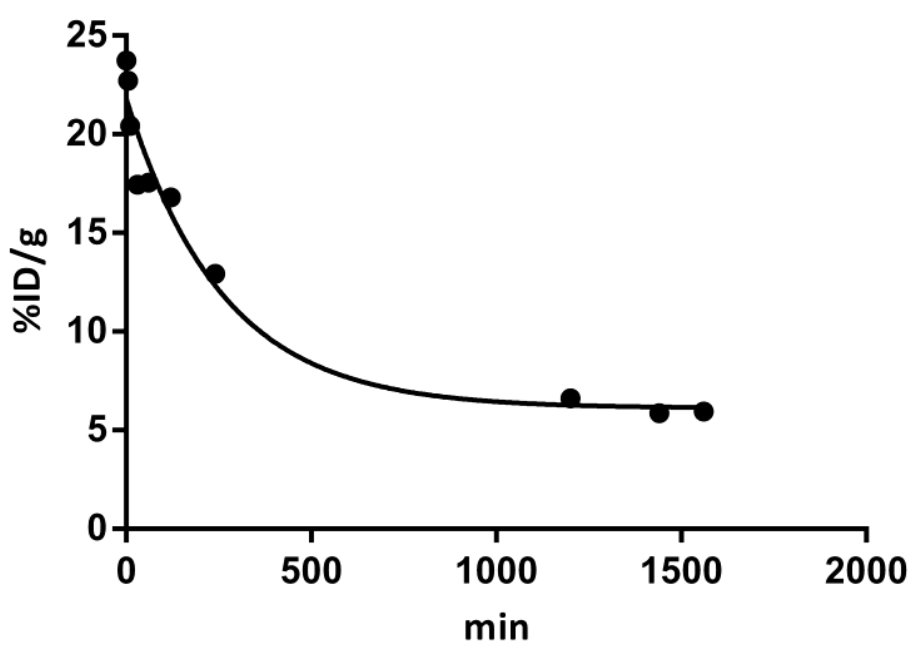

Figure 28: Blood clearance of ${ }^{99 \mathrm{~m}} \mathrm{Tc}$-Cetuximab

The blood clearance and half-life in blood were determined in a non-tumor bearing mouse for $\operatorname{lgG}_{1}$ ${ }^{99 \mathrm{~m}}$ Tc-Cetuximab (half-life $180 \mathrm{~min}, \mathrm{~N}=1$ ) by non-linear regression applying a of a one phase exponential decay fitting. A remaining activity of $6.1 \% \mathrm{ID} / \mathrm{g} \pm 0.89$ was determined as plateau for ${ }^{99 \mathrm{~m}} \mathrm{Tc}$-Cetuximab. 
Validation of the specific in vivo tumor visualization with ${ }^{99 m} T c$-Cetuximab

The specificity of the binding of ${ }^{99 \mathrm{~m}} \mathrm{Tc}$-Cetuximab to EGFR in vivo was validated by use of an ${ }^{99 \mathrm{~m}} \mathrm{Tc}$-isotype antibody. $15 \mu \mathrm{g}(98 \mathrm{pmol}, 103 \mathrm{MBq} \pm 14)$ of the radiolabeled ${ }^{99 \mathrm{~m}} \mathrm{Tc}$ isotype antibody were injected i.v. in MDA-MB-468 tumor bearing mice $(\mathrm{N}=3)$ and in vivo SPECT scans were performed $24 \mathrm{~h}$ post injection. The next day, the same mice received $15 \mu \mathrm{g}$ (98 pmol, $95 \mathrm{MBq} \pm 5$ ) of the radiolabeled ${ }^{99 \mathrm{~m}} \mathrm{Tc}$-Cetuximab i.v. and underwent in vivo SPECT scans $24 \mathrm{~h}$ post injection (Figure 29A). A significant tumor uptake of ${ }^{99 \mathrm{~m}} \mathrm{Tc}$-Cetuximab of $6.4 \% \mathrm{ID} / \mathrm{cm}^{3} \pm 2.0(p<0.05)$ was determined compared to unspecific tissue uptake (area on the contra lateral side, consisting presumably of muscle, fat and bone) of $0.6 \% \mathrm{ID} / \mathrm{cm}^{3} \pm 0.2$ (Figure 29B). The ${ }^{99 \mathrm{~m}} \mathrm{Tc}$-isotype antibody revealed a significant tumor uptake of $2.1 \% \mathrm{ID} / \mathrm{cm}^{3} \pm 0.1(\mathrm{p}<0.005)$ compared to unspecific tissue uptake of $0.75 \% \mathrm{ID} / \mathrm{cm}^{3} \pm 0.05$. Nevertheless, the tumor uptake of ${ }^{99 m}$ Tc-Cetuximab was significantly higher compared to the ${ }^{99 m}$ Tc-isotype $(p<0.05)$ concluding a specific uptake of ${ }^{99 \mathrm{~m}}$ Tc-Cetuximab to MDA-MB-468 tumors. Values for unspecific uptake of ${ }^{99 m} \mathrm{Tc}-\operatorname{lgG} 1$ and ${ }^{99 \mathrm{~m}} \mathrm{Tc}$-Cetuximab in tissues did not differ significantly. 


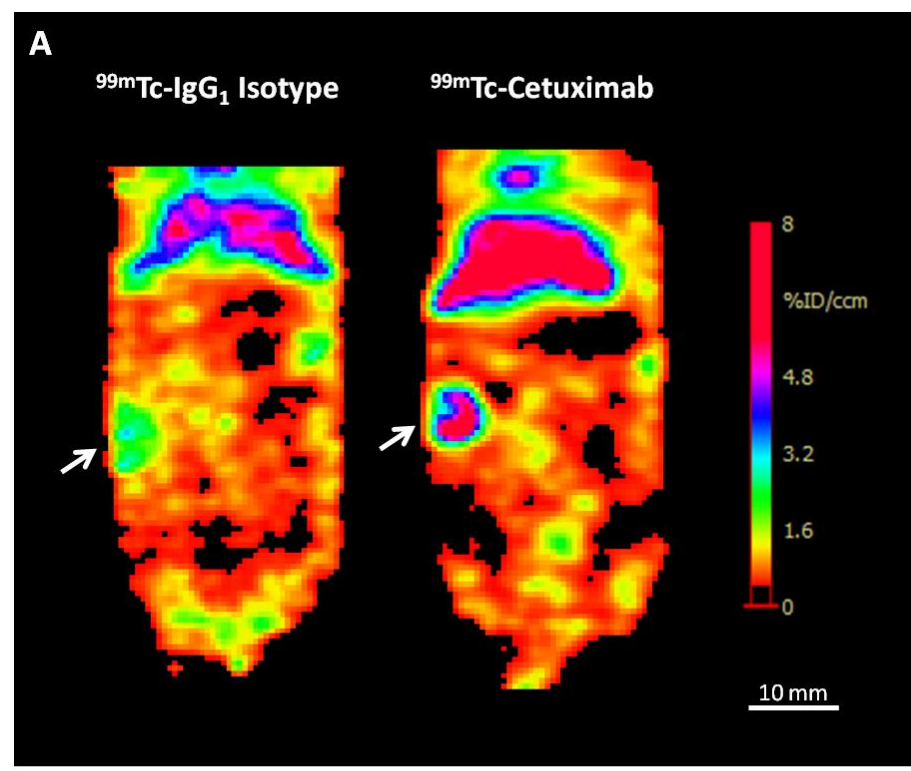

B

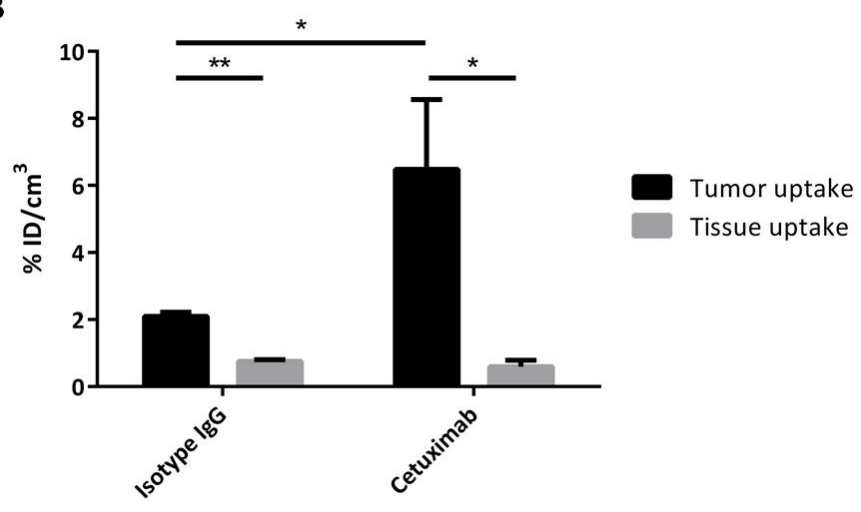

Figure 29: Uptake of ${ }^{99 m}$ Tc-Cetuximab to MDA-MB-468 tumors was specific

In vivo validation of specific binding of ${ }^{99 \mathrm{~m}}$ Tc-Cetuximab to EGFR by SPECT. MDA-MB-468 tumor bearing mice $(N=3)$ received $(A) 15 \mu \mathrm{g}(98 \mathrm{pmol}, 103 \mathrm{MBq} \pm 14)$ of the radiolabeled ${ }^{99 \mathrm{~m}}$ Tc-isotype antibody and (B) $15 \mu \mathrm{g}\left(98 \mathrm{pmol}, 95 \mathrm{MBq} \pm 5\right.$ ) of the radiolabeled ${ }^{99 \mathrm{~m}}$ Tc-Cetuximab i.v. on consecutive days. In vivo SPECT scans were performed $24 \mathrm{~h}$ post each injection. A significant tumor uptake of ${ }^{99 \mathrm{~m}} \mathrm{Tc}$-Cetuximab of $6.4 \% \mathrm{ID} / \mathrm{cm}^{3} \pm 2.0$ was determined compared to unspecific tissue uptake (area on the contra lateral side, consisting presumably of muscle, fat and bone) of $0.6 \% \mathrm{ID} / \mathrm{cm}^{3} \pm 0.2$. The ${ }^{99 \mathrm{~m}} \mathrm{Tc}$-isotype antibody revealed a significant tumor uptake of $2.1 \% \mathrm{ID} / \mathrm{cm}^{3} \pm 0.1$ compared to an unspecific tissue uptake of $0.75 \% \mathrm{ID} / \mathrm{cm}^{3} \pm 0.05$. The tumor uptake of ${ }^{99 \mathrm{~m}} \mathrm{Tc}$-Cetuximab was significantly higher compared to the ${ }^{99 \mathrm{~m}}$ Tc-isotype concluding a specific uptake of ${ }^{99 \mathrm{~m}}$ Tc-Cetuximab to MDA-MB-468 tumors. Unspecific tissue uptakes of both radiolabeled antibodies did not differ significantly. $360^{\circ}$ SPECT scans were performed in 10 frames with an acquisition of $180 \mathrm{sec}$ per frame; total scan time were 30 min. Tumor derived signals were segmented and compared to an area of the same volume of the contra lateral side. Data are shown as mean \pm SD in $\% \mathrm{ID} / \mathrm{cm}^{3}$. Tumor and tissue uptakes were compared applying a ratio paired t test. *: $p<0.05 ; * *$ : $p<0.005$. Bar represents $10 \mathrm{~mm}$. 
Visualization of tumors by in vivo SPECT and biodistribution analysis applying ${ }^{99 m}$ Tc-Cetuximab

After demonstrating specific tumor visualization of of high EGFR expressing MDA-MB468 tumors by the use of ${ }^{99 \mathrm{~m}} \mathrm{Tc}$-Cetuximab in combination with in vivo SPECT, the approach was adapted to the low EGFR expressing MDA-MB-231 tumors. A dose finding study figured out $10 \mu \mathrm{g}(65 \mathrm{pmol})$ as best concentration for tumor visualization applying multi-pinhole SPECT. In this study in vivo SPECT scans and ex vivo biodistribution analysis was performed on different cohorts.

Mice bearing the high and low EGFR expressing MDA-MB-468 and MDA-MB-231 tumors, respectively received each 65 pmol $(10 \mu \mathrm{g} ; 87 \mathrm{MBq} \pm 12){ }^{99 \mathrm{~m}} \mathrm{Tc}$-Cetuximab i.v. For biodistribution analysis two MDA-MB-468 tumor bearing mice received $65 \mathrm{pmol}$ $(10 \mu \mathrm{g} ; 82 \mathrm{MBq} \pm 4)^{99 \mathrm{~m}} \mathrm{Tc}-\operatorname{lgG}_{1}$ isotype for biodistribution analysis. In vivo SPECT scans (tumor volumes: $115.2 \mathrm{~mm}^{3} \pm 73.8 ; \mathrm{N}=10$ ) or ex vivo biodistribution analysis (tumor weights: $32 \mathrm{mg} \pm 20 ; \mathrm{N}=7$ ) of MDA-MB-468 tumor bearing mice were performed 24 post probe injection. In vivo SPECT scans revealed a high tumor uptake of ${ }^{99 \mathrm{~m}} \mathrm{Tc}$ Cetuximab with values of $5.5 \% \mathrm{ID} / \mathrm{cm}^{3} \pm 2.2$ compared to an unspecific tissue uptake (area of the contra lateral side) of $0.7 \% \mathrm{ID} / \mathrm{cm}^{3} \pm 0.2$ resulting in a tumor to tissue ratio of $7.8 \pm 3.2$ (Table 19). Besides the distinct tumor derived signal a high accumulation in the liver could be detected assuming hepatic excretion of ${ }^{99 \mathrm{~m}}$ Tc-Cetuximab. Furthermore, unspecific accumulation in the armpits of the forelegs suggest unspecific uptake in the lymph nodes, especially within the axial or brachial lymph nodes.

A high tumor uptake of ${ }^{99 \mathrm{~m}} \mathrm{Tc}$-Cetuximab of $14.6 \% \mathrm{ID} / \mathrm{g} \pm 6.9$ was determined ex vivo for MDA-MB-468 tumors compared to a remaining activity of $5.5 \%$ ID/g \pm 1.9 in the blood and an muscle uptake of $0.5 \% \mathrm{ID} / \mathrm{g} \pm 0.1$ resulting in tumor to blood and tumor to muscle ratios of $3.1 \pm 2.5$ and $30.7 \pm 18.3$, respectively (Figure 31 and Table 20).

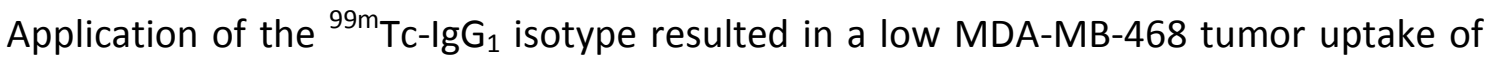
$1.1 \% \mathrm{ID} / \mathrm{g} \pm 0.2$ determined by ex vivo biodistribution analysis. Unspecific uptake to the muscle and remaining activity in the blood were comparable to ${ }^{99 \mathrm{~m}} \mathrm{Tc}$-Cetuximab (Figure 31 and Table 19). 
Table 19: In vivo determined tumor and tissue uptake of ${ }^{99 \mathrm{~m}} \mathrm{Tc}$-Cetuximab

\begin{tabular}{|c|c|c|c|c|c|}
\hline Tumor model & $\mathbf{N}$ & $\begin{array}{l}\text { Volume } \\
\mathrm{mm}^{3}\end{array}$ & $\begin{array}{c}\text { Tumor uptake } \\
\% \mathrm{ID} / \mathrm{cm}^{3}\end{array}$ & $\begin{array}{l}\text { Tissue uptake } \\
\% \mathrm{ID} / \mathrm{cm}^{3}\end{array}$ & $\begin{array}{l}\text { Tumor to } \\
\text { tissue ratio }\end{array}$ \\
\hline MDA-MB-468 & 10 & $115.2 \pm 73.8$ & $5.49 \pm 2.20$ & $0.74 \pm 0.25$ & $7.8 \pm 3.2$ \\
\hline MDA-MB-231 & 5 & $1247 \pm 1199$ & $2.13 \pm 0.37$ & $0.56 \pm 0.25$ & $4.3 \pm 1.8$ \\
\hline \multicolumn{6}{|c|}{$\begin{array}{l}\text { In vivo tumor and tissue uptakes were determined } 24 \mathrm{~h} \text { post }{ }^{99 \mathrm{~m}} \text { Tc-Cetuximab intravenous injection in } \\
\text { tumor bearing mice by in vivo SPECT scans. Volumes of tumor derived signals were displayed in } \mathrm{mm}^{3} \text {. } \\
\text { Tissue uptake was determined by segmentation of a region with the same size as the tumor containing } \\
\text { tissue only on the contra lateral side and used for determination of the in vivo tumor to tissue ratios. } \\
\text { Data are shown as mean } \pm \text { standard deviation in } \% \mathrm{ID} / \mathrm{cm}^{3} \text {. }\end{array}$} \\
\hline
\end{tabular}



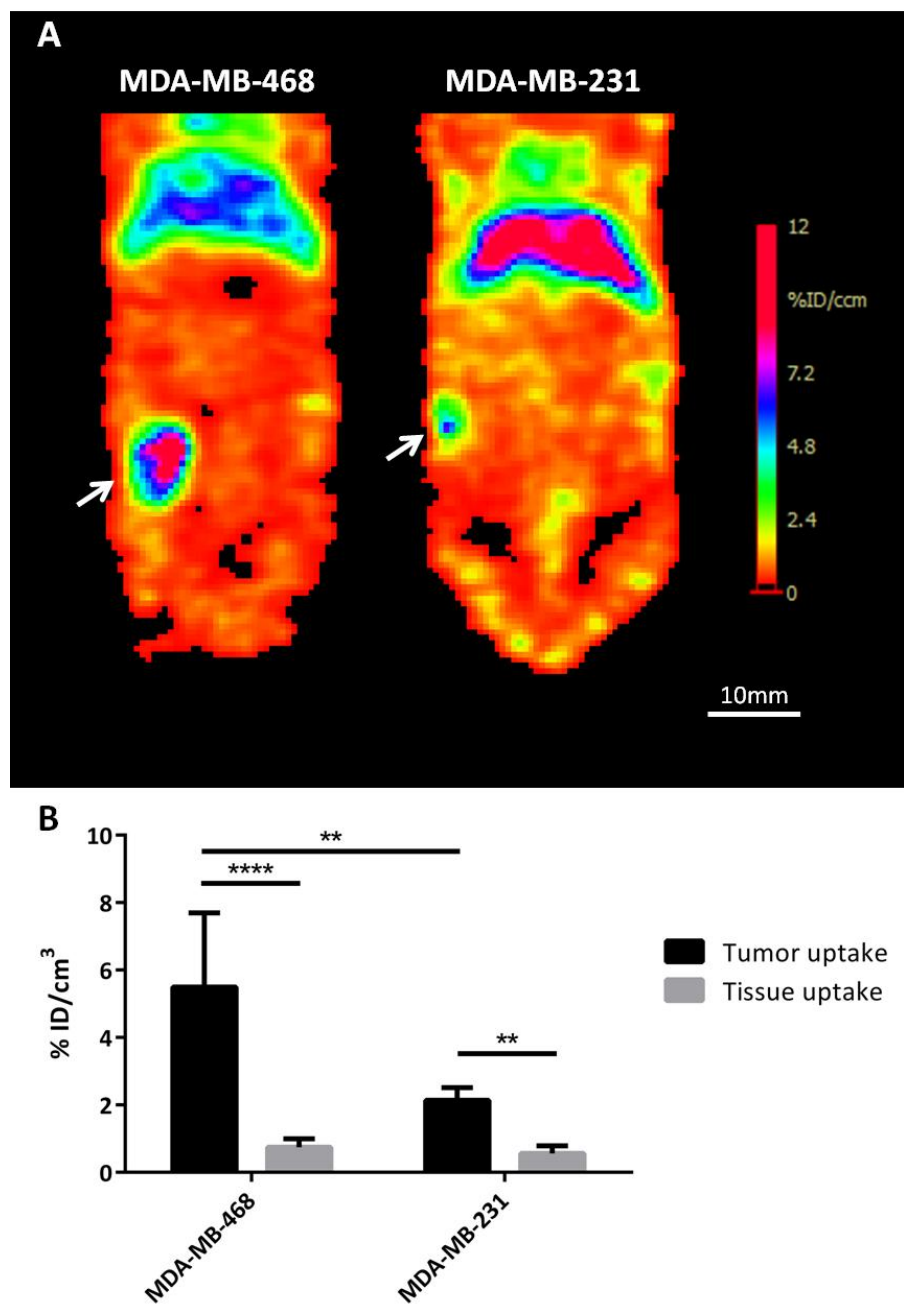

Figure 30: Tumor uptake of ${ }^{99 \mathrm{~m}}$ Tc-Cetuximab was dependent on EGFR expression

Mice bearing the high and low EGFR expressing MDA-MB-468 ( $N=10)$ and MDA-MB-231 ( $=5)$ tumors, respectively received $65 \mathrm{pmol}(10 \mu \mathrm{g} ; 87 \mathrm{MBq} \pm 12){ }^{99 \mathrm{~m}}$ Tc-Cetuximab i.v. (A) In vivo SPECT scans were performed 24 post injection and revealed a significant tumor uptake of ${ }^{99 \mathrm{~m}} \mathrm{Tc}$-Cetuximab of $5.5 \% \mathrm{ID} / \mathrm{cm}^{3} \pm 2.2$ and $2.1 \% \mathrm{ID} / \mathrm{cm}^{3} \pm 0.4$ to MDA-MB-468 and MDA-MB-231 tumors compared to tissue uptakes (area of the contra lateral side) of $0.7 \% \mathrm{ID} / \mathrm{cm}^{3} \pm 0.2$ (MDA-MB-468) and $0.6 \% \mathrm{ID} / \mathrm{cm}^{3} \pm 0.2$ (MDA-MB-231). Furthermore, the uptake to MDA-MB-468 tumors was significantly higher than to MDA-MB-231 tumors but unspecific tissue uptakes of ${ }^{99 \mathrm{~m}} \mathrm{Tc}$-Cetuximab did not differ significantly. A high accumulation in the liver could be detected, assuming hepatic excretion of ${ }^{99 \mathrm{~m}} \mathrm{Tc}$-Cetuximab. Also, accumulation in the armpits of the forelegs was detected suggesting unspecific uptake of ${ }^{99 \mathrm{~m}} \mathrm{Tc}$ Cetuximab to the lymph nodes. $360^{\circ}$ SPECT scans were performed in 10 frames with an acquisition of $180 \mathrm{sec}$ per frame; total scan time were $30 \mathrm{~min}$. Tumor derived signals were segmented and compared to an area of the same volume of the contra lateral side. Data are presented as mean \pm SD in $\% \mathrm{ID} / \mathrm{cm}^{3}$. Tumor and tissue uptakes were compared applying a ratio paired $t$ test. An unpaired two-tailed t test was used for comparison between MDA-MB-468 and MDA-MB-231. ${ }^{* *}: p<0.05$; $^{* * * *}: \mathrm{p}<0.0001$. 
Mice bearing low EGFR expressing MDA-MB-231 tumors underwent in vivo SPECT scans (tumor volumes: $1247 \mathrm{~mm}^{3} \pm 1199 ; \mathrm{N}=5$ ) or ex vivo biodistribution analysis (tumor weights: $164 \mathrm{mg} \pm 200 ; \mathrm{N}=8$ ) $24 \mathrm{~h}$ post injection. Compared to high EGFR expressing MDA-MB-468 tumors, in vivo SPECT scans revealed a relatively low tumor uptake of ${ }^{99 \mathrm{~m}} \mathrm{Tc}$-Cetuximab to MDA-MB-231 tumors of $2.1 \% \mathrm{ID} / \mathrm{cm}^{3} \pm 0.4$ compared to an unspecific tissue uptake (area of the contra lateral side) of $0.6 \% \mathrm{ID} / \mathrm{cm}^{3} \pm 0.2$ resulting in an in vivo tumor to tissue ratio of $4.4 \pm 1.8$ (Figure 30 and Table 19). Noteworthy, the MDA-MB-231 tumors were approx. 10 fold larger compared to MDAMB-468 tumors. Similar to the results obtained in MDA-MB-468 tumor bearing mice, a high accumulation in the liver and unspecific accumulations in the armpits of the forelegs were detected.

A tumor uptake of ${ }^{99 \mathrm{~m}} \mathrm{Tc}$-Cetuximab of $6.2 \% \mathrm{ID} / \mathrm{g} \pm 2.7$ was determined ex vivo for MDA-MB-231 tumors compared to a remaining activity of $5.2 \% \mathrm{ID} / \mathrm{g} \pm 1.3$ in the blood and an muscle uptake of $0.5 \% \mathrm{ID} / \mathrm{g} \pm 0.1$ resulting in tumor to blood and tumor to muscle ratios of $1.2 \pm 0.4$ and $11.2 \pm 4.4$, respectively (Figure 31 and Table 20).

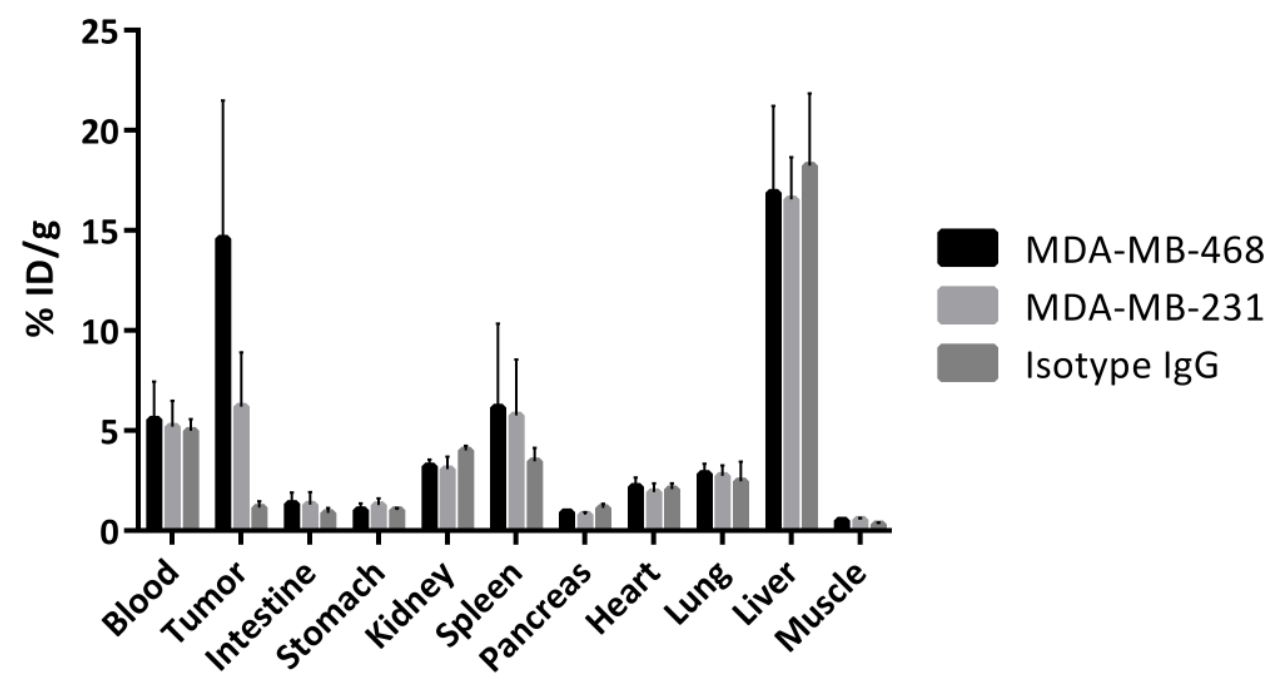

Figure 31: Ex vivo biodistribution analysis of ${ }^{99 \mathrm{~m}} \mathrm{Tc}$-Cetuximab in tumor bearing mice

Ex vivo biodistribution was carried out $24 \mathrm{~h}$ post i.v. injection of $65 \mathrm{pmol}(10 \mu \mathrm{g})$ in MDA-MB-468 $(\mathrm{N}=7)$ or MDA-MB-231 ( $\mathrm{N}=8)$ tumor bearing mice. The ${ }^{99 \mathrm{~m}} \mathrm{Tc}$-Isotype IgG was administered to MDA-MB-468 tumor bearing mice $(\mathrm{N}=2)$. The tumor uptake of ${ }^{99 \mathrm{~m}} \mathrm{Tc}$-Cetuximab was dependent on the EGFR expression level with values of $14.6 \% \mathrm{ID} / \mathrm{g} \pm 6.9$ for the high EGFR expressing MDA-MB-468 and $6.2 \% \mathrm{ID} / \mathrm{g} \pm 2.7$ for the low EGFR expressing MDA-MB-231 tumor. Administration of the isotype control IgG resulted in an low uptake of $1.1 \% \mathrm{ID} / \mathrm{g} \pm 0.3$ in a MDA-MB-468 tumor. Both antibodies had an equally high remaining activity in the blood $(5.5 \% \mathrm{ID} / \mathrm{g} \pm 1.9)$ and were removed from the body via hepatic excretion (liver uptake: $16.8 \% \mathrm{ID} / \mathrm{g} \pm 4.3$ ). Data are presented as mean \pm SD in \% ID/g. 
Table 20: Ex vivo determined tumor and tissue uptake of ${ }^{99 \mathrm{~m}}$ Tc-Cetuximab

\begin{tabular}{|c|c|c|c|c|c|c|c|c|}
\hline \multirow[b]{2}{*}{ Tumor model } & \multirow[b]{2}{*}{ Antibody } & \multirow[b]{2}{*}{$\mathbf{N}$} & \multirow[b]{2}{*}{$\begin{array}{l}\text { Tumor weight } \\
\text { mg }\end{array}$} & \multicolumn{3}{|c|}{ Uptake } & \multicolumn{2}{|c|}{ Ratio } \\
\hline & & & & $\begin{array}{l}\text { Tumor } \\
\% \text { ID/g }\end{array}$ & $\begin{array}{l}\text { Blood } \\
\% \text { ID } / g\end{array}$ & $\begin{array}{l}\text { Muscle } \\
\% \text { ID/g }\end{array}$ & Tumor to blood & Tumor to tissue \\
\hline MDA-MB-468 & ${ }^{99 m}$ Tc-Cetuximab & 7 & $32 \pm 20$ & $14.60 \pm 6.89$ & $5.54 \pm 1.90$ & $0.50 \pm 0.12$ & $3.1 \pm 2.5$ & $30.7 \pm 18.3$ \\
\hline MDA-MB-231 & ${ }^{99 m}$ Tc-Cetuximab & 8 & $164 \pm 200$ & $6.19 \pm 2.71$ & $5.20 \pm 1.28$ & $0.54 \pm 0.08$ & $1.2 \pm 0.4$ & $11.2 \pm 4.4$ \\
\hline MDA-MB-468 & ${ }^{99 m} \mathrm{Tc}^{-I g G_{1}}$ Isotype & 2 & $22 \pm 7$ & $1.14 \pm 0.22$ & $4.99 \pm 0.41$ & $0.32 \pm 0.06$ & $0.2 \pm 0.03$ & $3.4 \pm 0.04$ \\
\hline
\end{tabular}

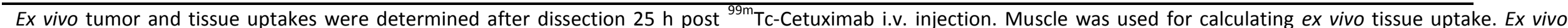
uptakes are expressed as mean \pm SD in $\% \mathrm{ID} / \mathrm{g}$. 


\subsubsection{Tumor visualization with anti EGFR nanobody ${ }^{99 m}$ Tc-D10}

Similar to the experiments with ${ }^{99 m}$ Tc-Cetuximab the orthotopic MDA-MB-468 and MDA-MB-231 human mammary carcinoma mouse models were used to assess the efficacy of anti-EGFR nanobody ${ }^{99 \mathrm{~m}} \mathrm{Tc}$-D10 to detect mammary tumors with a different EGFR expression level. First, the blood clearance was determined in non-tumor bearing mice with a serum half-life of $4.9 \mathrm{~min}$ with a plateau of $0.26 \% \mathrm{ID} / \mathrm{g} \pm 0.24$ was determined for ${ }^{99 \mathrm{~m}} \mathrm{Tc}$-D10 allowing imaging from approximately $45 \mathrm{~min}$ post injection (Figure 32).

anti-EGFR nanobody ${ }^{99 \mathrm{~m}}$ Tc-D10

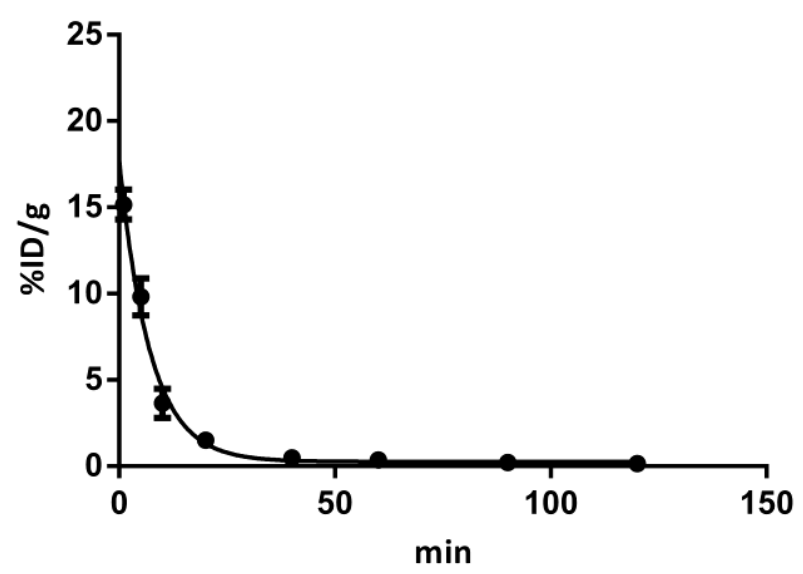

Figure 32: Blood clearance of ${ }^{99 \mathrm{~m}} \mathrm{Tc}-\mathrm{D} 10$

The blood clearance and half-life in blood were determined in non-tumor bearing mice for anti-EGFR nanobody ${ }^{99 \mathrm{~m}} \mathrm{Tc}-\mathrm{D} 10$ (half-life of $4.9 \mathrm{~min} ; \mathrm{N}=2$ ) by non-linear regression applying a of a one phase exponential decay fitting. A remaining activity of $0.26 \% \mathrm{ID} / \mathrm{g} \pm 0.24$ was determined as plateau for the nanobody ${ }^{99 \mathrm{~m}} \mathrm{TC}-\mathrm{D} 10$.

Mice bearing high EGFR expressing MDA-MB-468 (Figure 20 and Figure 21) tumors with a mean volume of $56.5 \mathrm{~mm}^{3} \pm 21.2\left(\mathrm{~N}=5\right.$; range $\left.35.1-89.4 \mathrm{~mm}^{3}\right)$ were used for in vivo tumor detection with anti-EGFR nanobody ${ }^{99 \mathrm{~m}}$ Tc-D10 by SPECT. All MDA-MB-468 tumors were detected reliably by in vivo SPECT scans 45 min after administration of 17 pmol anti-EGFR nanobody ${ }^{99 \mathrm{~m}} \mathrm{Tc}$-D10 with a tumor to tissue (area of the contra lateral side) ratio of $42.8 \pm 27.0$ (Figure 33D). Ex vivo analyses of biodistribution in MDA-MB468 tumor bearing mice revealed a tumor uptake of $1.3 \%$ ID/g \pm 0.27 (Figure 33B) with a tumor to blood ratio of $5.4 \pm 1.4$ and a tumor to tissue ratio of $12.5 \pm 7.3$ (Figure 33C, 
Table 21). An uptake of $192.5 \% \mathrm{ID} / \mathrm{g} \pm 30.9$ and $3.4 \% \mathrm{ID} / \mathrm{g} \pm 1.4$ of ${ }^{99 \mathrm{~m}} \mathrm{Tc}-\mathrm{D} 10$ to the kidneys and the liver, respectively was determined ex vivo (Figure 33B). MDA-MB-231 tumors with a very low expression of EGFR showed no detectable uptake of the antiEGFR nanobody ${ }^{99 \mathrm{~m}} \mathrm{TC}$-D10 (Figure 34). Neither in vivo SPECT nor ex vivo biodistribution analyses demonstrate an uptake of ${ }^{99 \mathrm{~m}} \mathrm{Tc}-\mathrm{D} 10$ in MDA-MB-231 tumors with a mean volume of $124 \mathrm{~mm}^{3} \pm 102\left(\mathrm{~N}=5\right.$; range $21.1-247.5 \mathrm{~mm}^{3}$ ) (Figure 33A and C). The lack of ${ }^{99 \mathrm{~m}} \mathrm{Tc}-\mathrm{D} 10$ to accumulate within the tumor indicated no unspecific binding or tumor uptake of the probe.

A

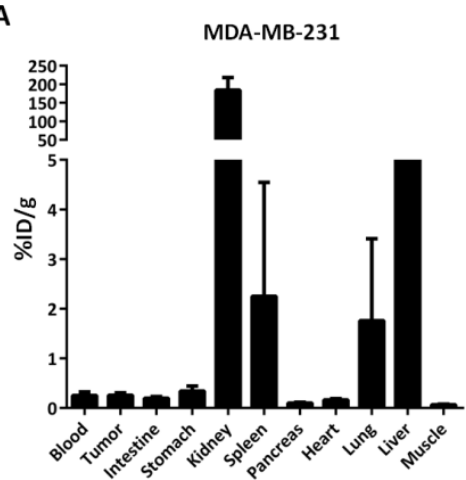

C

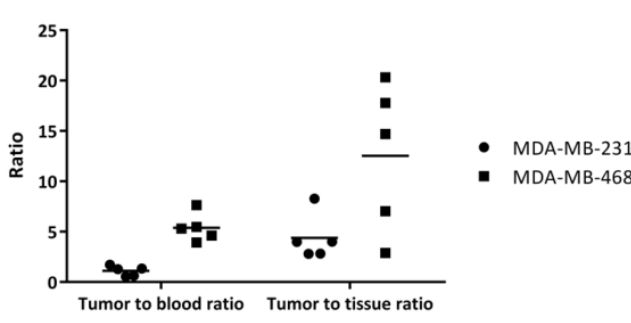

B
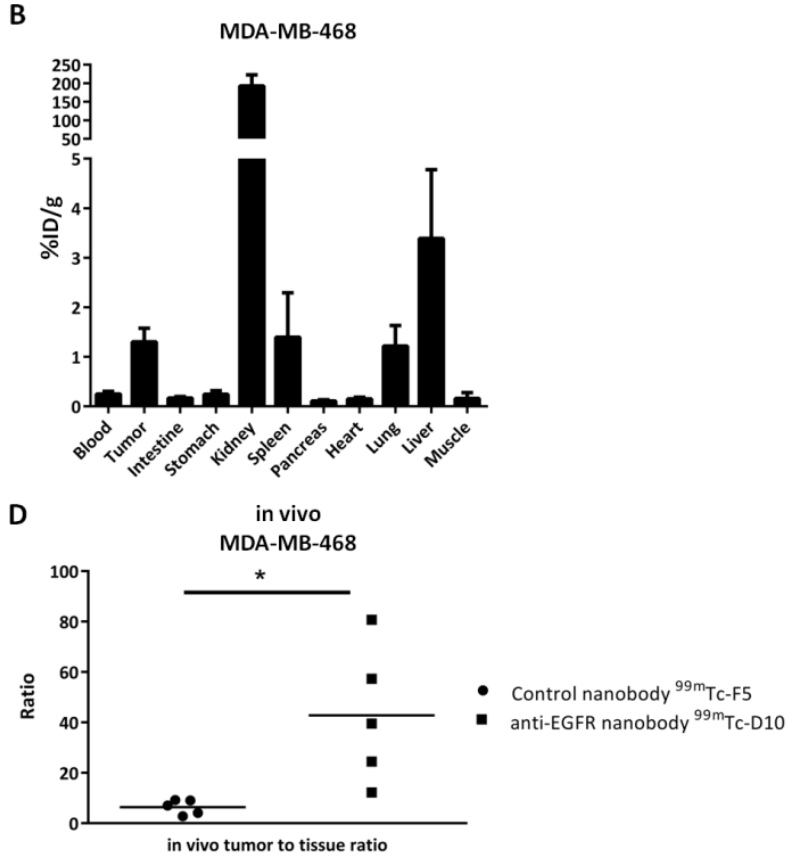

Figure 33: Tumor uptake of ${ }^{99 \mathrm{~m}} \mathrm{Tc}$-D10 was dependent on EGFR expression

MDA-MB-231 and MDA-MB-468 tumor bearing mice received $17 \mathrm{pmol}(2.3 \mathrm{MBq})$ anti-EGFR nanobody ${ }^{99 \mathrm{~m}} \mathrm{TC}-\mathrm{D} 10$ and $17 \mathrm{pmol}$ of irrelevant nanobody ${ }^{99 \mathrm{~m}} \mathrm{TC}-\mathrm{F5}$ (2.4 MBq) intravenously. Ex vivo biodistribution analysis 100 min post injection of anti-EGFR nanobody ${ }^{99 m}$ Tc-D10 are shown in (A) MDA-MB-231 (N = 5) and (B) MDA-MB-468 (N=5) tumor bearing mice. (C) Ex vivo tumor to blood ratios and tumor to tissue (muscle) ratios of nanobody ${ }^{99 \mathrm{~m}} \mathrm{Tc}-\mathrm{D} 10$ are presented. (D) In vivo SPECT scans were performed in MDAMB-468 tumor bearing mice 45 min post injection of either nanobody ${ }^{99 \mathrm{~m}} \mathrm{Tc}$-D10 or control nanobody ${ }^{99 \mathrm{~m}} \mathrm{TC}$-F5. The tumor volume was segmented and compared to an equally sized contra lateral region. *P $<0.05$, unpaired two-tailed t-Test, for in vivo tumor to tissue ratio nanobody ${ }^{99 \mathrm{~m}} \mathrm{Tc}$-D 10 vs. control nanobody ${ }^{99 \mathrm{~m}} \mathrm{TC}-\mathrm{F} 5$. 


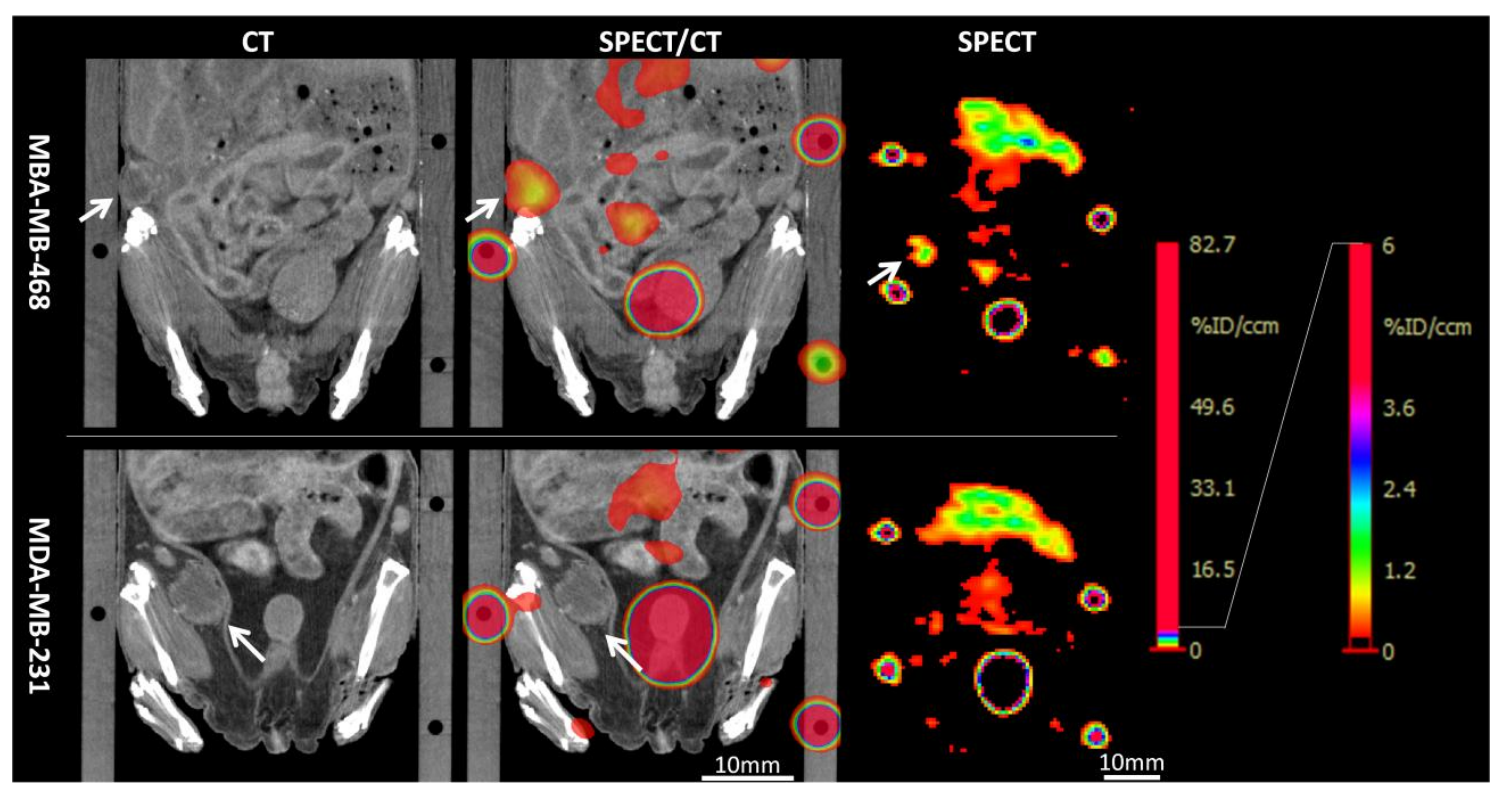

Figure 34: Tumor visualization of mammary MDA-MB-468 and MDA-MB-231 tumors

Representative SPECT and CT images of mice are shown that bear an orthotopic MDA-MB-468 tumor (volume $64 \mathrm{~mm}^{3}$ ) or a MDA-MB-231 tumor (volume $129 \mathrm{~mm}^{3}$ ) and received $17 \mathrm{pmol}(2.4-3.1 \mathrm{MBq}$ ) anti-EGFR nanobody ${ }^{99 \mathrm{~m}}$ Tc-D10 by i.v. administration. SPECT was performed 45 min post injection. Contrast-enhanced CT using Ultravist370 (left panel) and SPECT scans (right panel) were performed on different modalities and images were aligned by hand according to ${ }^{99 \mathrm{~m}}$ Tc-pertechnetate landmarks (middle panel). Tumors are indicated by white arrows. A high tumor accumulation with a low background was achieved with anti-EGFR nanobody ${ }^{99 \mathrm{~m}} \mathrm{Tc}-\mathrm{D} 10$ in the MDA-MB-468 tumor model (upper panel). No accumulation of nanobody ${ }^{99 \mathrm{~m}}$ Tc-D10 was detectable in the MDA-MB-231 tumor model with a very low EGFR expression (lower panel). 
Table 21: Tumor and tissue uptake of ${ }^{99 \mathrm{~m}} \mathrm{Tc}-\mathrm{D} 10$ and ${ }^{99 \mathrm{~m}} \mathrm{Tc}$-Cetuximab

\begin{tabular}{|c|c|c|c|c|c|c|c|c|c|c|c|}
\hline \multirow[b]{2}{*}{ Tumor model } & \multirow[b]{2}{*}{ Antibody } & \multirow[b]{2}{*}{$\mathrm{N}$} & \multirow[b]{2}{*}{$\begin{array}{c}\text { Tumor size } \\
\mathrm{mm}^{3}\end{array}$} & \multirow[b]{2}{*}{$\begin{array}{c}\text { Tumor } \\
\text { weight } \\
\text { mg }\end{array}$} & \multicolumn{2}{|c|}{ Tumor uptake } & \multicolumn{2}{|c|}{ Tissue uptake } & \multicolumn{3}{|c|}{ Ratios } \\
\hline & & & & & $\begin{array}{l}\text { ex vivo } \\
\% \text { ID/g }\end{array}$ & $\begin{array}{l}\text { in vivo } \\
\% \mathrm{ID} / \mathrm{cm}^{3}\end{array}$ & $\begin{array}{l}\text { ex vivo } \\
\% \mathrm{ID} / \mathrm{g}\end{array}$ & $\begin{array}{l}\text { in vivo } \\
\% \mathrm{ID} / \mathrm{cm}^{3}\end{array}$ & $\begin{array}{l}\text { Tumor to } \\
\text { blood } \\
\text { ex vivo }\end{array}$ & $\begin{array}{l}\text { Tumor to } \\
\text { tissue } \\
\text { ex vivo }\end{array}$ & $\begin{array}{l}\text { Tumor to } \\
\text { tissue } \\
\text { in vivo }\end{array}$ \\
\hline A431 & $\begin{array}{c}{ }^{99 \mathrm{~m}} \mathrm{Tc}- \\
\text { Cetuximab }\end{array}$ & 5 & $\begin{array}{c}40.0 \pm \\
38.9\end{array}$ & $\begin{array}{c}50 \pm \\
34\end{array}$ & $6.5 \pm 2.0$ & $2.1 \pm 0.8$ & $0.43 \pm 0.1$ & $0.5 \pm 0.2$ & $0.9 \pm 0.3$ & $15.2 \pm 4.1$ & $8.4 \pm 7.2$ \\
\hline A431 & ${ }^{99 \mathrm{~m}}$ Tc-D10 & 6 & $\begin{array}{c}26.6 \pm \\
16.7\end{array}$ & $\begin{array}{c}43 \pm \\
30\end{array}$ & $2.3 \pm 0.7$ & $1.0 \pm 0.6$ & $0.14 \pm 0.1$ & $0.03 \pm 0.01$ & $12.1 \pm 3.5$ & $25.6 \pm 18.8$ & $36.2 \pm 20.9$ \\
\hline MDA-MB-468 & ${ }^{99 \mathrm{~m}}$ Tc-D10 & 5 & $\begin{array}{c}56.5 \pm \\
21.2\end{array}$ & $\begin{array}{c}94 \pm \\
51\end{array}$ & $1.3 \pm 0.3$ & $0.6 \pm 0.2$ & $0.15 \pm 0.1$ & $0.02 \pm 0.02$ & $5.4 \pm 1.4$ & $12.5 \pm 7.3$ & $42.8 \pm 27.1$ \\
\hline MDA-MB-231 & ${ }^{99 \mathrm{~m}} \mathrm{Tc}-\mathrm{D} 10$ & 5 & $\begin{array}{c}124.0 \pm \\
102.3\end{array}$ & $\begin{array}{c}251 \pm \\
205\end{array}$ & $0.25 \pm 0.1$ & n.a. & $0.06 \pm 0.02$ & $0.02 \pm 0.01$ & $1.1 \pm 0.5$ & n.a. & n.a. \\
\hline
\end{tabular}

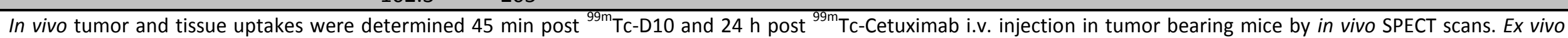

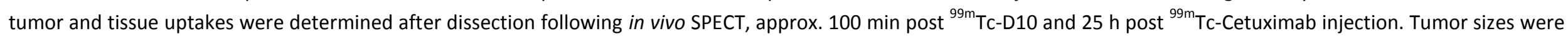

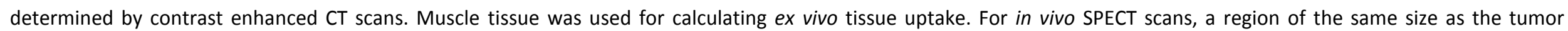

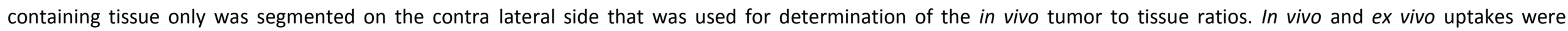
expressed as percentage of injected dose per cubic centimeter $\left(\% \mathrm{ID} / \mathrm{cm}^{3}\right)$ and gram $(\% \mathrm{ID} / \mathrm{g})$, respectively. Data are shown as mean \pm standard deviation. $\mathrm{n} . \mathrm{a} .=$ not applicable. 


\subsubsection{Visualization of very small tumor lesions}

Mice bearing human A431 epidermoid subcutaneous tumor xenografts of small size with a mean tumor volume of $26 \mathrm{~mm}^{3}\left(\mathrm{~N}=6\right.$; range $7-54 \mathrm{~mm}^{3}$, Table 21) received the anti-EGFR nanobody ${ }^{99 \mathrm{~m}} \mathrm{Tc}-\mathrm{D} 10$ that accumulated in the tumor and generated a high tumor to tissue (muscle) contrast. In vivo SPECT revealed a high contrast within the tumor with tumor to tissue (area of the contra lateral side) ratios of $36.2 \pm 20.9$ in 5 out of 6 animals (Figure 35D and Figure 36). Besides tumor derived signals, the kidneys and the liver were clearly visible by SPECT imaging. The specific tumor uptake was validated in SPECT scans in the same mice prior to ${ }^{99 m} \mathrm{Tc}-\mathrm{D} 10$ administration by applying the control nanobody $\mathrm{F} 5$ that showed in all mice no tumor uptake (Figure 35D and Figure 36). Following in vivo SPECT imaging, the uptake of anti-EGFR nanobody ${ }^{99 \mathrm{~m}}$ Tc-D10 in A431 tumors was confirmed by ex vivo biodistribution analyses in all 6 animals with a tumor uptake of $2.27 \% \mathrm{ID} / \mathrm{g} \pm 0.68$ (Figure $35 \mathrm{~A}$ ) resulting in a tumor to blood ratio of $12.1 \pm 3.5$ and a tumor to tissue (muscle) ratio of $25.6 \pm 18.8$ (Figure 35C and Table 21). Kidneys and liver had uptakes of $160.7 \%$ ID/g \pm 17.9 and $2.1 \%$ ID/g \pm 0.3 , respectively. This results in tumor to kidney and tumor to liver ratios of $0.014 \pm 0.004$ and $1.1 \pm 0.4$, respectively, demonstrating that the nanobody ${ }^{99 \mathrm{~m}} \mathrm{Tc}-\mathrm{D} 10$ was excreted via the kidney (Table 21). The absent signals in the SPECT scans in one tumor bearing animal out of six can be explained by the very small tumor volume of $7 \mathrm{~mm}^{3}$ and the limited resolution of the used multi-pinhole collimators being above $2 \mathrm{~mm}$, which do not resolve those small structures.

The efficacy of in vivo tumor detection with anti-EGFR nanobody ${ }^{99 \mathrm{~m}} \mathrm{Tc}-\mathrm{D} 10$ was compared to the $\operatorname{lgG}_{1}{ }^{99 \mathrm{~m}} \mathrm{Tc}$-Cetuximab that was used to visualize $\mathrm{A} 431$ tumor lesions with comparable mean tumor volumes of $40 \mathrm{~mm}^{3}\left(\mathrm{~N}=5\right.$; range $\left.7-90 \mathrm{~mm}^{3}\right) 45 \mathrm{~min}$ post injection. In vivo SPECT scans with ${ }^{99 \mathrm{~m}} \mathrm{Tc}$-Cetuximab showed no uptake of ${ }^{99 \mathrm{~m}} \mathrm{Tc}$ Cetuximab in the small tumor lesions at the early time point. However, in a second scan $24 \mathrm{~h}$ post injection, a tumor uptake in 3 out of 5 animals could be detected with a tumor to tissue ratio of $8.4 \pm 7.2$, much less compared to results obtained with the nanobody ${ }^{99 \mathrm{~m}}$ Tc-D10 (36.2 \pm 20.9$)$ (Figure 35D). This discrepancy can be explained by a slow blood clearance and a serum half-life of approximately $180 \mathrm{~min}$ of ${ }^{99 \mathrm{~m}} \mathrm{Tc}$ - 
Cetuximab compared to the short serum half-life of ${ }^{99 \mathrm{~m}} \mathrm{Tc}-\mathrm{D} 10$ being less than $5 \mathrm{~min}$. As with ${ }^{99 \mathrm{~m}} \mathrm{Tc}-\mathrm{D} 10$, the absent signals in SPECT scans in A431 tumors of two out of five mice receiving ${ }^{99 m}$ Tc-Cetuximab, might be explained by the very small volumes of these tumors, ranging from 7 to $12 \mathrm{~mm}^{3}$ with a diameter of $2 \mathrm{~mm}$ being below the resolution of the SPECT system.

Ex vivo biodistribution analysis revealed a tumor uptake of $6.5 \% \mathrm{ID} / \mathrm{g} \pm 2.0$ (Figure 35B) for ${ }^{99 \mathrm{~m}} \mathrm{Tc}$-Cetuximab resulting in a tumor to blood ratio of only $0.9 \pm 0.3$ and a tumor to tissue ratio of $15.2 \pm 4.1$ (Figure $35 \mathrm{C}$ ). This is only one tenth of the tumor to blood ratio and half of the tumor to tissue ratio obtained with the anti-EGFR nanobody ${ }^{99 \mathrm{~m}} \mathrm{Tc}-\mathrm{D} 10$.
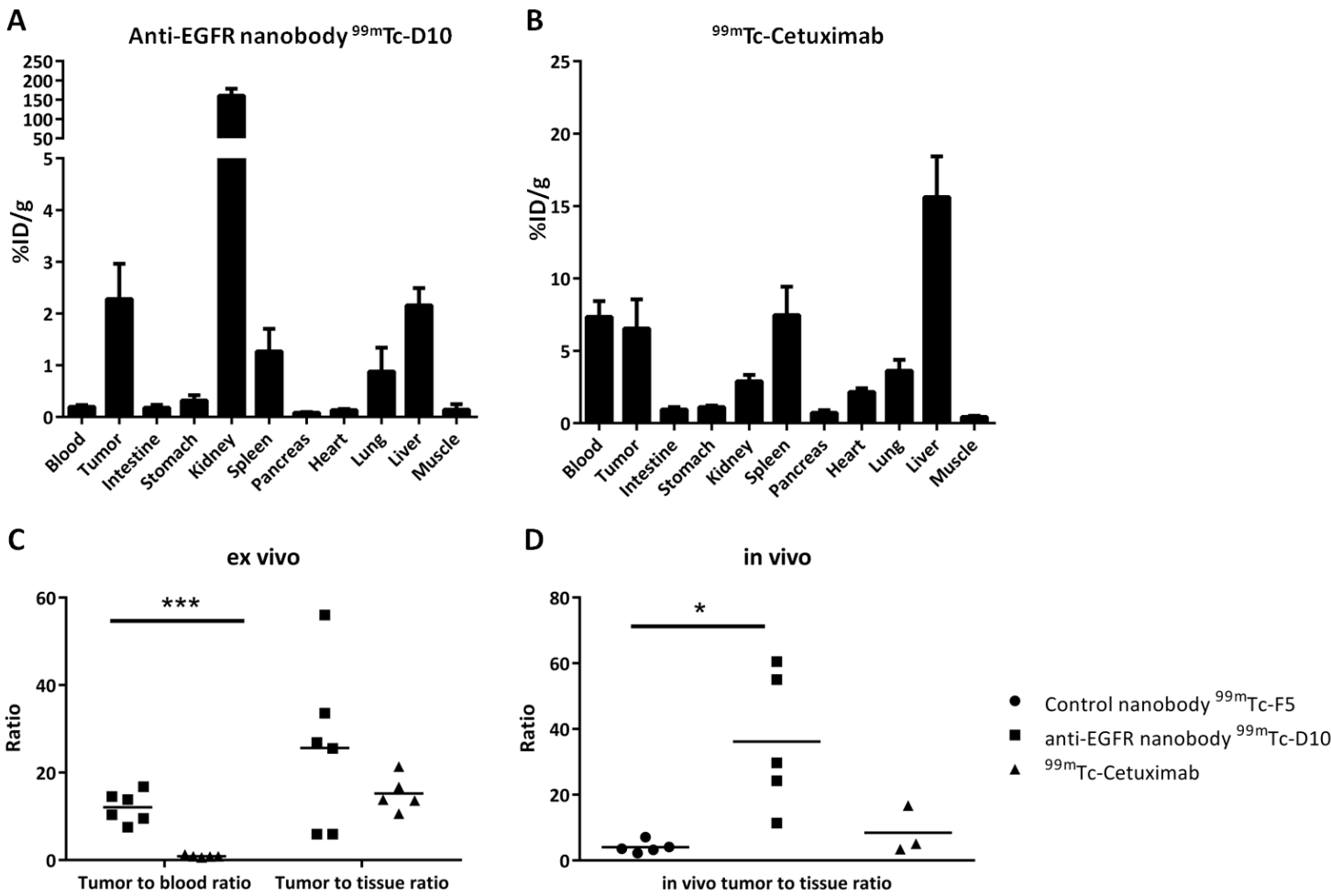

Figure 35: Uptake of anti-EGFR nanobody ${ }^{99 \mathrm{~m}} \mathrm{Tc}$-D10 in A431 tumors

A431 tumor bearing mice received either $17 \mathrm{pmol}(2.6-5.1 \mathrm{MBq})$ radiolabeled anti-EGFR nanobody ${ }^{99 m}$ Tc-D10 $(\mathrm{N}=6)$ or $9 \mathrm{pmol}(9.4-15.6 \mathrm{MBq}){ }^{99 \mathrm{~m}} \mathrm{Tc}-$ Cetuximab $(\mathrm{N}=5)$ intravenously. Ex vivo biodistribution (A) 100 min post injection of anti-EGFR nanobody ${ }^{99 m}$ Tc-D10 and (B) 25 h post injection of ${ }^{99 \mathrm{~m}}$ Tc-Cetuximab are shown. (C) Ex vivo tumor to blood ratios and tumor to tissue (muscle) ratios after nanobody ${ }^{99 \mathrm{~m}}$ Tc-D10 application (100 min post injection) compared to ${ }^{99 \mathrm{~m}}$ Tc-Cetuximab (25 $\mathrm{h}$ post injection) are presented. (D) In vivo SPECT scans were performed 45 min post nanobody ${ }^{99 m}$ Tc-D10 and irrelevant nanobody ${ }^{99 \mathrm{~m}} \mathrm{Tc}-\mathrm{F} 5$ injection and $24 \mathrm{~h}$ post ${ }^{99 \mathrm{~m}} \mathrm{Tc}$-Cetuximab injection. The area of the tumor was segmented and compared to an equally sized contra lateral region. Ratios were calculated as ratio of tumor uptake and tissue uptake. Due to the resolution of $2 \mathrm{~mm}$ of the SPECT system, one tumor of the cohort receiving ${ }^{99 \mathrm{~m}} \mathrm{Tc}$-D10 and two tumors of the cohort receiving ${ }^{99 \mathrm{~m}} \mathrm{Tc}$-Cetuximab could not be detected in vivo. ${ }^{*} \mathrm{P}<0.05$, unpaired two-tailed $\mathrm{t}$-Test, for in vivo tumor to tissue ratio: nanobody ${ }^{99 \mathrm{~m}} \mathrm{Tc}-$ D10 vs. irrelevant nanobody ${ }^{99 \mathrm{~m}} \mathrm{Tc}-\mathrm{F} 5$ and nanobody ${ }^{99 \mathrm{~m}} \mathrm{Tc}-\mathrm{D} 10$ vs. ${ }^{99 \mathrm{~m}} \mathrm{Tc}-$ Cetuximab. ${ }^{* * *} \mathrm{P}<0.001$, unpaired two-tailed t-Test, for ex vivo tumor to blood ratio: nanobody ${ }^{99 \mathrm{~m}}$ Tc-D10 vs. ${ }^{99 \mathrm{~m}} \mathrm{Tc}$-Cetuximab. 


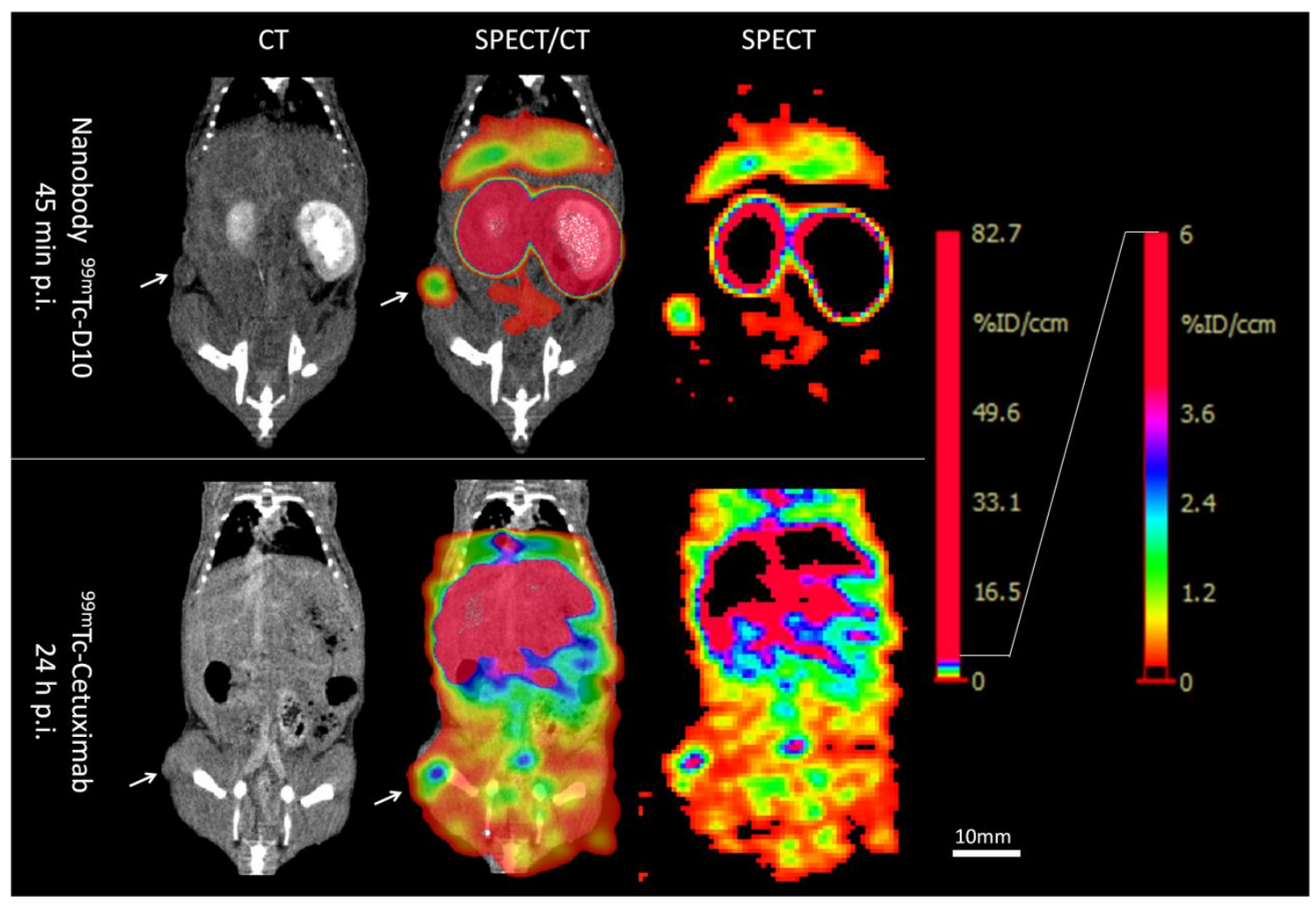

Figure 36: In vivo tumor visualization of small human A431 tumors with ${ }^{99 \mathrm{~m}}$ Tc-D10

Representative SPECT images are shown of A431 tumor bearing mice that received $17 \mathrm{pmol}$ (3.3 MBq; tumor volume $22 \mathrm{~mm}^{3}$ ) radiolabeled anti-EGFR nanobody ${ }^{99 \mathrm{~m}} \mathrm{Tc}-\mathrm{D} 10$ or $9 \mathrm{pmol}$ (10.5 MBq; tumor volume $\left.16 \mathrm{~mm}^{3}\right)^{99 \mathrm{~m}}$ Tc-Cetuximab i.v. SPECT imaging was performed 45 min post ${ }^{99 \mathrm{~m}}$ Tc-D10 and $24 \mathrm{~h}$ post ${ }^{99 \mathrm{~m}}$ TcCetuximab administration. Contrast-enhanced CT (left panel) and SPECT scans (right panel) were performed on different modalities and images were aligned by hand according to ${ }^{99 \mathrm{~m}} \mathrm{Tc}$-pertechnetate landmarks ( $<30 \mathrm{kBq}$ ) (middle panel). Tumors are indicated by white arrows. Note, that a high tumor accumulation with a low background was achieved with anti-EGFR nanobody ${ }^{99 \mathrm{~m}}$ Tc-D10. 


\section{Discussion}

The detection of tumors in an early phase of tumor development is an important achievement to improve the overall prognosis of the patient. Besides accurate information of tumor load and spread, the retrieval of the expression of biomarkers on the tumor cell surface at the earliest time point is a prerequisite for a successful targeted therapeutic approach. In this study, functional SPECT imaging with the antiEGFR nanobody ${ }^{99 m}$ Tc-D10 and the anti-EGFR antibody ${ }^{99 m}$ Tc-Cetuximab targeting the human tumor biomarker EGFR were shown to be promising approaches in the preclinical setting in order to acquire information on tumor localization as well as expression of tumor associated proteins in vivo. The generation and characterization of novel anti-Kv10.1 nanobodies targeting the human voltage-gated potassium channel Kv10.1 was a first step in the development of an in vivo anti-Kv10.1 based specific tumor detection by applying multi-pinhole SPECT.

\subsection{Generation of specific anti-Kv10.1 nanobodies C4 and D9}

In this study, two anti-Kv10.1 nanobodies, C4 and D9, were identified as promising candidates for the detection of Kv10.1 in vivo and proven to specifically detect Kv10.1 in transfected HEK cells and the recombinant fusion protein in different in vitro assays like ELISA, IP and Western blotting. The ability of nanobodies C4 and D9 to specifically bind to the potassium channel Kv10.1 was proven by IP in Kv10.1 and empty plasmid transfected HEK cells and validated by the detection of the precipitate with a different anti-Kv10.1 antibody, 9391. The here determined affinities of the nanobodies C4 and D9 to $\mathrm{H} 1 \mathrm{X}$ in the nanomolar range are comparable to many other published nanobodies targeting less challenging epitopes like receptors on the cellular surface $[50,96-98]$. An interpretation of the results obtained by flow cytometry on intact cells to detect the native potassium channel Kv10.1 by the nanobodies C4 and D9 was not possible yet with Kv10.1 transfected HEK cells due to a low transfection efficiency. This experiment has to be repeated on cells with an improved transfection efficiency and a more suitable reporter system. 
The generation of selective and specific antibodies against ion channels for diagnosis and treatment of hematological malignancies is a very important yet challenging task. Unlike conventional targets used for antibody-based tumor detection like receptors on the cellular surface, ion channels usually cannot be purified for immunization, because membrane proteins require a lipid environment to maintain their native structure [99, 100]. Further immunization techniques like the injection of whole cells or parts of cell membranes expressing high amounts of the antigen of interest do not apply for ion channels due to the low expression level on the cell surface [97, 99]. Therefore, linear peptides like the recombinant fusion protein $\mathrm{H} 1 \mathrm{X}$ are generally used for immunization to overcome this issue. Many antibodies were raised against linear epitopes of ion channels mapping a short sequence of the channel of interest $[13,20,101]$. Since research is directed towards the identification of novel therapeutic antibodies targeting ion channels, the peptide sequences represent a part of the E3 loop in voltage-gated potassium channels, i.e. the third extracellular domain which contains the pore region. However, only very few antibodies are capable to bind to the native channel [100]. A concept to avoid the immunization with a linear epitope is the use of virus-like particles to capture and concentrate structurally intact membrane proteins within lipoparticles direct from the cell surface without mechanical disruption or detergents [99].

The use of nanobodies targeting ion channels might evade the problem with the immunization with a linear epitope because of the small size and close proximity of the CDRs. Recently, a nanobody targeting the potassium channel Kv1.3 was presented by Ablynx, the leading nanobody company [102]. The use of nanobodies, like the antiKv10.1 nanobodies C4 and D9, for the targeting of ion channels is straightforward, since nanobodies, due to their small size of only $2.5 \times 4 \mathrm{~nm}$, can bind sterical demanding epitopes like pore regions that remain inaccessible for antibodies $[50,98]$. A further advantage of nanobodies is the possibility of easy modification strategies by molecular cloning and subsequent expression in bacterial cultures. Compared to cell cultures which are needed for the expression of antibodies, the costs of nanobody expression in $E$. coli are reduced to a mere fraction. 


\subsection{Is Kv10.1 a suitable target for molecular imaging?}

The specific detection and visualization of a tumor requires the generation of sufficient contrast to distinguish between tumor and surrounding healthy tissue. The suitability of a newly developed imaging probe for tumor visualization can be initially assessed in vitro by flow cytometry. Assessing the binding capacities of the nanobody intended for in vivo use on the tumor cells that will be used for tumor cell implantation in mice, as was performed for the targeting of EGFR, allows an estimation of the suitability of the nanobody for imaging. In the case of EGFR, A431 and MDA-MB-468 cells with a receptor density of more than $1 \times 10^{6}$ EGFR per cell were reliably detected with D10 and ${ }^{99 m}$ Tc-D10 $[94,95]$. No binding, i.e. no increase of the MFI compared to baseline, could be detected after incubation of D10 with MDA-MB-231 cells, that were reported to express approx. $2 \times 10^{5}$ EGFR per cell [94]. No such information is available for the number Kv10.1 ion channels expressed in the membrane of tumor cells. So far, the expression of Kv10.1 was assessed by quantitative Real-time polymerase chain reaction ( $q R T-P C R$ ) and quantified in pTracer-Kv10.1 transfected Chinese hamster ovary $(\mathrm{CHO})$ cells, sarcoma tumor cell lines as well as control transfected $\mathrm{CHO}$ cells as negative control. For the transfected $\mathrm{CHO}$ cells, 39,000 copies (mRNA of KCHN1) per 1000 cells were published, i.e. on average 39 copies per cell compared 0.3 copies per 1000 cells in the negative control [20]. For sarcoma tumor cells, that were described as clearly positive, 140 copies per 1000 cells were reported [20]. However, the transcription of a gene does not have to necessarily correlate with the amount of expressed protein or the functional Kv10.1 potassium channel on the cell surface.

A solution to overcome a relatively weak target protein expression is to increase the affinity by generation of a biparatopic nanobody [103]. In contrast to bivalent IgG antibodies that consist of two antigen-binding sites, a nanobody as monomer can only bind to a single paratope. Usually, antibodies have a higher affinity compared to nanobodies, because the bivalency generates avidity, i.e. the accumulated strength of multiple affinities or functional affinity $[89,104]$. Therefore, the generation of a bivalent nanobody that binds two paratopes in the pore of a tetrameric ion channel appears very promising. Due to the fact, that the pore is assembled out of four Kv10.1 subunits, a dimer, trimer or tetramer of C4 or D9 could be generated. 
The suitability of Kv10.1 as imaging target depends further on the protein expression within the tumor. The work with the anti-EGFR nanobody and antibody on EGFR expressing tumors revealed intratumoral heterogeneity as a challenge for in vivo imaging. In recent years, Kv10.1 was associated with tumor development and described as potential tumor biomarker with robust expression [11-13, 20, 26, 29, 105108]. A real advantage of using a probe targeting Kv10.1 is the absent expression of Kv10.1 in healthy tissue, that could result in a perfect tumor to tissue contrast, assuming no Kv10.1 potassium channels are present on the cells of healthy tissue and available anti-Kv10.1 nanobodies with high affinity towards the ion channel.

For now, the question if Kv10.1 is a suitable imaging target remains unanswered. On the one hand the low expression of the potassium channel on the surface of cells will be challenging for a successful in vivo tumor detection. On the other hand the absent Kv10.1 expression in the surrounding tissue could provide an optimal contrast. However, applying an engineered nanobody with a high affinity towards Kv10.1, a tumor visualization by targeting Kv10.1 could be conceivable.

\subsection{Nanobodies as versatile tools in biology and medicine}

Nanobodies in general and more particularly the anti-Kv10.1 nanobodies C4 and D9 can also be used as versatile tools for various applications in molecular biology and histology like conventional antibodies as well. However, the straightforward production in $E$. coli and an easy modification on gene level allow an efficient and economic production. For the anti-Kv10.1 nanobodies C4 and D9, it is planned to establish a toolkit for fluorescence microscopy, flow cytometry and IHC within the next months. Therefore, the nanobodies will be engineered with a free C-terminal cysteine that allows site-specific labeling of the thiol function, allowing the labeling of maleimide-conjugated fluorescent dyes and enzymes such as AP or HRP. The benefit of the use of the nanobodies as a platform will be the efficient production in a large scale, straightforward purification via IMAC and less time for repeated quality controls. 
A further potential of nanobodies was recently shown by the development of novel diagnostic markers for the detection of EGFR, Her2 and carcinoembryonic antigen on tumor biopsies using nanobodies conjugated to quantum dots [56, 109].

Finally, nanobodies hold great promise for therapy of various diseases. In 2014, a clinical trial was granted by the European Medial Agency for the treatment of human respiratory syncytial virus with a nebulized trivalent nanobody (EMEA-001553-PIP0113). In oncology, nanobodies are investigated for the treatment of solid cancers as bispecific molecules, that bind to a target on a tumor and simultaneously engaging components of the immune system, or as carriers for cytotoxic drugs to reduce side effects $[50,110,111]$. The anti-Kv10.1 nanobodies C4 and D9 have to be evaluated for a possible therapeutic effect. The binding in the pore of Kv10.1 might lead to a selective blockade of the potassium channel, making a therapeutic application conceivable.

\subsection{Nanobodies and antibodies as tools for molecular imaging}

The application of antibodies and nanobodies in vivo for the detection and visualization of tumors applying in vivo SPECT was assessed on well characterized EGFR expressing tumor models with the recently described anti-EGFR nanobody ${ }^{99 m}$ Tc-D10 as well as the anti-EGFR antibody ${ }^{99 m}$ Tc-Cetuximab to establish a SPECT imaging infrastructure in order to be able to adapt the gained knowledge to the application of anti-Kv10.1 nanobodies [96].

\subsubsection{Tumor visualization with ${ }^{99 \mathrm{~m}} \mathrm{Tc}$-Cetuximab by SPECT}

Applying the radiolabeled full $\operatorname{lgG}_{1}$ anti-EGFR antibody ${ }^{99 m}$ Tc-Cetuximab for the visualization of human MDA-MB-231 and MDA-MB-468 mammary carcinomas with different EGFR expression levels, tumor uptakes of $2.1 \% \mathrm{ID} / \mathrm{cm}^{3} \pm 0.4$ and $5.5 \% \mathrm{ID} / \mathrm{cm}^{3}$ \pm 2.2 compared to tissue uptakes of $0.6 \% \mathrm{ID} / \mathrm{cm}^{3} \pm 0.2$ and $0.7 \% \mathrm{ID} / \mathrm{cm}^{3} \pm 0.2$ were determined $24 \mathrm{~h}$ post i.v. injection by in vivo SPECT, respectively. Thus, the in vivo determined tumor to tissue ratio was $7.8 \pm 3.2$ for MDA-MB-468 and $4.4 \pm 1.8$ for MDA-MB-231. The relations in the tumor and tissue uptakes of ${ }^{99 m}$ Tc-Cetuximab to the 
two different mammary tumors were confirmed by ex vivo biodistribution analyses. Uptakes of $14.6 \% \mathrm{ID} / \mathrm{g} \pm 6.9$ and $6.2 \% \mathrm{ID} / \mathrm{g} \pm 2.7$ of ${ }^{99 \mathrm{~m}} \mathrm{Tc}$-Cetuximab to MDA-MB-468 and MDA-MB-231 tumors were determined, respectively, compared to tissue (muscle) uptakes of $0.5 \% \mathrm{ID} / \mathrm{g} \pm 0.1$ for both tumor bearing cohorts. Such high tumor uptakes of ${ }^{99 \mathrm{~m}}$ Tc-Cetuximab as observed with MDA-MB-468 tumors match published data showing values of $15-20 \% \mathrm{ID} / \mathrm{g}$ in tumor tissue for the same and A431 tumor models with a comparable EGFR expression level [112, 113]. Unspecific binding of ${ }^{99 \mathrm{~m}} \mathrm{Tc}$ Cetuximab was assessed by use of an ${ }^{99 \mathrm{~m}} \mathrm{Tc}-\operatorname{lgG}_{1}$ isotype control antibody. A significant uptake of ${ }^{99 m}$ Tc-isotype to MDA-MB-468 tumors with values of $2.1 \% \mathrm{ID} / \mathrm{cm}^{3} \pm 0.1$ in comparison to a tissue uptake of $0.75 \% \mathrm{ID} / \mathrm{cm}^{3} \pm 0.05$ was determined by in vivo SPECT scans. In vivo SPECT scans with ${ }^{99 \mathrm{~m}}$ Tc-Cetuximab in the same animals on consecutive days showed a significantly higher tumor uptake of ${ }^{99 \mathrm{~m}} \mathrm{Tc}$-Cetuximab $\left(6.4 \% \mathrm{ID} / \mathrm{cm}^{3} \pm\right.$ 2.0). These results prove the specific uptake of ${ }^{99 m} \mathrm{Tc}$-Cetuximab to MDA-MB-468 tumors. The unspecific tumor uptake of ${ }^{99 \mathrm{~m}} \mathrm{Tc}-\lg G_{1}$ was unfortunately not assessed on MDA-MB-231 tumor bearing mice, thus prohibiting a direct comparison. However, the in vivo tumor uptake of ${ }^{99 \mathrm{~m}} \mathrm{Tc}$-Cetuximab to MDA-MB-231 tumors with values of $2.1 \% \mathrm{ID} / \mathrm{cm}^{3} \pm 0.4$ might not be considered as specific, compared to the ${ }^{99 \mathrm{~m}} \mathrm{Tc}$-isotype uptake of $2.1 \% \mathrm{ID} / \mathrm{cm}^{3} \pm 0.1$ to MDA-MB-468 tumors. The relatively high tumor uptake of the ${ }^{99 m}$ Tc-isotype is remarkable because no binding to MDA-MB-468 cells, i. e. no increase of the MFI compared to baseline, was observed by flow cytometry with the isotype antibody. An explanation for the uptake of the isotype antibody in the tumor tissue might be the enhanced permeation and retention (EPR) effect of larger macromolecules such as polymers or proteins [114-117]. EPR is a form of passive drug targeting due to the molecular weight of the molecule. This phenomenon occurs on the basis of different physiological properties of tumor tissue compared to normal tissue, since tumors perform angiogenesis which is connected with extensive vascularization to provide sufficient supply of nutrients and oxygen to the tumor [116]. The endothelial cells of these newly formed blood vessels are highly fenestrated compared to most other capillaries, thus allowing an easier diffusion of macromolecules from the blood pool to the tumor compared to healthy tissue [116, 117]. Additionally, an impaired lymphatic system of the tumors compared to healthy tissue prevents retention of the diffused macromolecules leading to an enrichment in 
the tumor [116]. Macromolecules with a molecular weight above $20 \mathrm{kDa}$ are prone to the EPR effect and the probability of this effect increases with increasing mass [115, 117]. Thus, the EPR effect applies most likely for full IgG antibodies with a molecular weight of approximately $150 \mathrm{kDa}$ and is diametric to a specific tumor targeting with full antibodies. An unspecific accumulation of the imaging probe hampers the use for a specific visualization and targeting, albeit this effect might be desirable for the treatment of tumor lesions.

Another challenge for the visualization of tumors with radiolabeled antibodies is the observation of a relatively high background activity after $24 \mathrm{~h}$ as shown in this study with uptakes of approx. $0.6 \% \mathrm{ID} / \mathrm{g}$ and $6 \% \mathrm{ID} / \mathrm{g}$ to muscle and blood, respectively and organ uptakes between 1 and 5\% ID/g. This relatively high background seen with ${ }^{99 \mathrm{~m}} \mathrm{Tc}$ Cetuximab has two disadvantages. The majority of the antibody is distributed throughout the body which reduces the contrast for in vivo imaging. Another issue is the generation of high signals in vivo in lymph nodes, especially in the axillary and superficial nodes as observed with ${ }^{99 \mathrm{~m}} \mathrm{Tc}$-Cetuxiumab and ${ }^{99 \mathrm{~m}} \mathrm{Tc} \operatorname{lgG}^{1}$ as well as with radiolabeled BSA. Own preliminary results show that parts of the injected BSA were cleared via the kidneys and via the liver accompanied with distinct signals in the axiallary and superficial lymph nodes $24 \mathrm{~h}$ post i.v. injection (data not shown). This can be explained by the drainage of unbound proteins in the interstitial fluid (the lymph) via lymph capillaries and vessels ending in lymph nodes which are finally emptied in the subclavian veins [118-120]. This unspecific accumulation of imaging probes in lymph nodes can be misleading if the detection of metastases, which often accumulate in axillary lymph nodes, is desired [120-122].

In this study, a serum half-life of approx. $3 \mathrm{~h}$ was determined for ${ }^{99 \mathrm{~m}} \mathrm{Tc}$-Cetuximab. The long circulation of the antibody in the blood pool, can be explained by the relatively large size of the $\operatorname{lgG}_{1}{ }^{99 m} \mathrm{Tc}$-Cetuximab, $152 \mathrm{kDa}$, and its removal from the body via hepatic excretion, manifested by a high liver uptake of approx. $20 \%$ ID/g , and the recycling process of antibodies afforded by the neonatal Fc receptor (FcRn) that is expressed in the cells of the liver and gut as well as in adult endothelia cells [89]. The FcRn binds the Fc portion of IgGs and become internalized by endocytosis. The acidic endosomes release the internalized $\lg G$ at the cell surface to the basic milieu of the blood and thereby prevent the IgG from lysosomal degradation [123, 124]. This long 
serum long half-life is detrimental for a tracer for tumor detection because it prevents a diagnostic tumor visualization shortly after injection of the radiolabeled probe. In this study, in vivo SPECT scans were started $24 \mathrm{~h}$ post i.v. ${ }^{99 \mathrm{~m}} \mathrm{Tc}$-Cetuximab injection. Since all molecules in this study were labeled with the isotope technetium-99m with a halflife of $6.01 \mathrm{~h}$, high amounts of radioactivity had to be injected to retain sufficient activity to the time of imaging after 4 half-lives. This issue can of course be circumvented by the use of other radionuclides with longer half-lives such as indium111 with a half-life of 2.8 days. Another drawback of a full IgG as diagnostic tracer for tumor visualization is the overall aim to achieve an application in man. Therefore, such a long lag time between injection and imaging appears not very convenient. Different concepts such as 'Pretargeting', involving a separate injection of an unlabeled cold antibody that is detected by a radiolabeled hapten are under clinical investigation [125-129].

\subsubsection{Application of anti-EGFR nanobody ${ }^{99 \mathrm{~m}}$ Tc-D10}

The application of the small-sized anti-EGFR nanobody ${ }^{99 \mathrm{~m}}$ Tc-D10 with a molecular weight of $15.5 \mathrm{kDa}$, proved that ${ }^{99 \mathrm{~m}} \mathrm{Tc}-\mathrm{D} 10$ is suitable to detect small tumor lesions expressing EGFR with high specificity and high contrast shortly after administration. These features are mandatory for tracers to enable tumor detection at earliest time points to improve the overall prognosis for the patient. The anti-EGFR nanobody ${ }^{99 \mathrm{~m}} \mathrm{Tc}$ D10 showed a tumor uptake even in very small A431 and MDA-MB-468 tumor lesions of up to $7 \mathrm{~mm}^{3}$ with an uptake of $2.18 \% \mathrm{ID} / \mathrm{g}$ already $100 \mathrm{~min}$ post injection as determined by ex vivo biodistribution analysis. In vivo SPECT imaging failed to detect very small lesions with a diameter below $2.5 \mathrm{~mm}$ corresponding to a volume of less than $15 \mathrm{~mm}^{3}$ since the multi-pinhole SPECT system used here only provides a resolution of above $2 \mathrm{~mm}$ illustrating the limitation of the imaging hardware. The fast blood clearance with a half-life in blood of only $4.9 \mathrm{~min}$ and a very low plateau of $0.26 \% \mathrm{ID} / \mathrm{g} \pm 0.24$ of the remaining activity in the blood pool allowed an early detection of EGFR expressing already after 45 min tumor lesions in vivo with an excellent contrast with a tumor to tissue ratio of up to 60 . 
Nanobodies are subject of investigation for several years and many studies were published targeting EGFR or HER2 overexpressing tumors with diameters of approximately $0.5-1 \mathrm{~cm}[7,53]$ or volumes of above $100 \mathrm{~mm}^{3}$ with tumor weights far beyond $100 \mathrm{mg}[7,8,53,130]$. These nanobodies have comparable affinities in the nanomolar range for their target receptors and were cleared within minutes to hours from the blood pool. The overall A431 tumor uptake values determined here in these study were 2-3 fold higher in vivo and ex vivo than the published ones obtained with the anti-EGFR nanobodies $[7,8,53]$. However, the reported blood activity (ex vivo) and tissue uptake (in vivo) were also higher, resulting in a comparable high contrast for the anti-EGFR nanobody ${ }^{99 \mathrm{~m}}$ Tc-D10 in vivo and high tumor to blood ratios ex vivo $[7,8,53]$. In contrast, in this study the EGFR on very small tumor lesions with weights below $100 \mathrm{mg}$ was targeted by ${ }^{99 \mathrm{~m}} \mathrm{Tc}-\mathrm{D} 10$. It is known that most tumors originate as small avascular structures and have to establish their own tumor derived blood vessels to grow beyond a few millimeters [131, 132]. Thus, tumors of small size might not to have high amounts of vasculature with fenestrated blood vessels that allow a quick extravasation of the nanobody and thereby a higher accumulation of probes within the tumor as observed in larger tumors [112, 113, 131-135]. A reason for the higher tumor uptake of previously published results using nanobodies might be the larger diameter $(0.5-1 \mathrm{~cm})$ or volume $\left(100-600 \mathrm{~mm}^{3}\right)$ of tumors used in these studies, leading to better vascularization of the tumor with more fenestrated blood vessels $[7,8]$. Others have shown that A431 tumors with a volume of at least $100 \mathrm{~mm}^{3}$ contain many small and mostly immature blood vessels that were homogenously distributed throughout the tumor [136]. Otherwise, an inverse relationship between tumor uptake and tumor weight was reported for EGFR targeting in A431 tumors with different anti-EGFR nanobodies [7]. The tendency of decreasing tumor uptake of nanobodies with increasing tumor weight was observed in tumors with weights ranging from $120 \mathrm{mg}$ up to around $800 \mathrm{mg}$. This effect can be explained on one hand by intratumoral heterogeneity, i.e. that different tumor cells of a tumor show distinct morphological and phenotypic profiles, including gene expression, metabolism and cellular morphology [137-139]. On the other hand the diameter of the blood vessels becomes narrower from the periphery to the center and might reduce the amount of nanobody reaching the center [136]. A third explanation might be the formation of necrotic 
tissue in the center that might prohibit binding of the nanobody. In contrast, in this study an increasing tumor uptake of nanobody ${ }^{99 \mathrm{~m}} \mathrm{Tc}-\mathrm{D} 10$ with increasing tumor volumes was observed, since small tumor lesions that might lack a tumor derived vasculature with fenestrated blood vessels were chosen for visualization [131, 132]. However, the observations made in this study and published data highlight the challenges in targeting and visualization of such small tumors.

This issue was further manifested by targeting of EGFR with ${ }^{99 m}$ Tc-Cetuximab in our setting resulting in a relatively low overall A431 tumor uptake of $6.5 \% \mathrm{ID} / \mathrm{g}$ compared to tumor uptakes of up to $20 \%$ ID/g observed by others $[112,113]$. The full $\operatorname{IgG}_{1}{ }^{99 \mathrm{~m}} \mathrm{Tc}$ Cetuximab led to a significantly lower tumor to blood ratio compared to nanobody ${ }^{99 m}$ Tc-D10, despite a more than 10 fold higher affinity towards EGFR (0.5 nM) [140]. Similarly, the ex vivo tumor to blood ratio was significantly impaired $(P<0.01)$ for ${ }^{99 \mathrm{~m}}$ Tc-Cetuximab after $25 \mathrm{~h}$ compared to ${ }^{99 \mathrm{~m}} \mathrm{Tc}-\mathrm{D} 10$ after $100 \mathrm{~min}$.

The anti-EGFR nanobody ${ }^{99 \mathrm{~m}}$ Tc-D10 was found to be predominantly removed by renal excretion as typical for small proteins with a molecular weight of $15 \mathrm{kDa}[7,8,10,141]$, a value under the $60 \mathrm{kDa}$ threshold of glomerular filtration in the kidneys [142-144]. A high kidney uptake of approx. $150 \% \mathrm{ID} / \mathrm{g}$ of ${ }^{99 \mathrm{~m}} \mathrm{Tc}-\mathrm{D} 10$ was observed and has previously been reported for many other nanobodies [7, 8, 47, 53, 130, 145-147]. The renal uptake is mediated by the Low Density Lipoprotein receptor-related protein 2 (LRP2 or megalin) in the proximal tubular cells [53]. Megalin is known to recover proteins from the urine by interaction on cationic domains via endocytosis or transcytosis to prevent proteinuria, i.e. the presence of an excess of serum proteins in the urine $[148,149]$. This unspecific accumulation might be problematic for imaging tumor lesions in close proximity to the kidneys and for the use of the nanobody as carrier for therapeutic applications such as the conjugation of beta emitters to nanobodies for radioimmunotherapy since the kidneys are very radiosensitive organs [150]. A possibility to reduce this renal uptake is the coinjection of cationic or polycationic amino acids or succinylated gelatine (gelofusine) [53, 142-144]. Recently, it was reported that the removal of the C-terminal 6xHis tag from an anti-PSMA nanobody also markedly reduced its renal uptake and could be combined with gelofusine and lysine to decrease the renal uptake below $4 \% \mathrm{ID} / \mathrm{g}$ at $3 \mathrm{~h}$ post injection [151]. A recently developed approach is the modification of a purified protein by a Sortase $A$ 
mediated exchange of the $6 x \mathrm{His}$ tag to a chelator of choice [152-154]. This transpepdidase reaction requires a cleavage site (LPETG motif) for Sortase $A$ in the nanobody, that can be easily introduced in the vector, upstream of the 6xHis tag and of the functionalized oligoglycine. Sortase A cleaves an amide bond in the cleavage motif that can be attacked by a primary amine of the oligoglycine [153]. This method would allow the immobilized metal affinity chromatography (IMAC) purification via the $6 x$ His tag of the nanobody and a subsequent functionalization for radiolabeling. However, in this study, the 6xHis tag of the nanobodies was used for radiolabeling. In the case of technetium-99m labeling, a polylysine peptide conjugated with HyNic could be introduced with the oligoglycine, allowing a site specific labeling of the nanobody.

High tumor to tissue ratios were obtained in vivo and ex vivo for ${ }^{99 m}$ Tc-D10 which is needed to define the tumor from the surrounding tissue. The variations of the ex vivo and in vivo tumor to tissue ratios were quite large compared to the data of ex vivo biodistribution analysis. The uptake of ${ }^{99 \mathrm{~m}} \mathrm{Tc}$-D10 in the muscle of A431 tumor bearing mice was very low with $0.14 \% \mathrm{ID} / \mathrm{g} \pm 0.1$, however, in regard to the low uptake values a distinct variance was observed ranging from $0.05 \% \mathrm{ID} / \mathrm{g}$ to $0.29 \% \mathrm{ID} / \mathrm{g}$. This variance was highlighted after calculation of the ratio causing the variations in the ex vivo and in vivo tumor to tissue ratios. For ${ }^{99 \mathrm{~m}} \mathrm{Tc}$-Cetuximab, this effect was less pronounced, since the muscle uptake was 3 -fold higher and varied less with $0.43 \% \mathrm{ID} / \mathrm{g} \pm 0.08$ compared to ${ }^{99 \mathrm{~m}} \mathrm{Tc}$-D10. Less variation of the uptake of ${ }^{99 \mathrm{~m}} \mathrm{Tc}$-Cetuximab in the muscle might be explained by the late time point of the in vivo SPECT scan at $24 \mathrm{~h}$ post ${ }^{99 \mathrm{~m}} \mathrm{Tc}$-Cetuximab injection and subsequent biodistribution. The variation of the ratios did not interfere with image interpretation and analysis since sufficient contrast between tumor and muscle was provided. A poor contrast would be achieved close to organs with an unspecific uptake similar or higher than the tumor uptake, making tumor visualization difficult to impossible.

\subsection{Summary and Conclusion}

In this project, a panel of ten individual anti-Kv10.1 nanobodies was generated by phage display and subsequent screening on the fusion protein H1X. Molecular 
characterization of the binding properties revealed two clones, C4 and D9, detecting specifically Kv10.1 transfected HEK cells and the fusion protein H1X.

For a later in vivo tumor visualization applying the anti-Kv10.1 nanobodies C4 and D9, a clinical SPECT system was set up and the infrastructure for the handling and housing of radioactive animals was established. This allowed the in vivo tumor visualization on EGFR expressing tumors with the clinically applied anti-EGFR antibody ${ }^{99 \mathrm{~m}} \mathrm{Tc}$-Cetuximab and demonstrated as proof of principle the successful application of multi-pinhole SPECT. Tumors expressing high levels of EGFR and a volume above $100 \mathrm{~mm}^{3}$ were reliably detected by in vivo SPECT, whereas tumors with a relatively low EGFR expression showed a tumor uptake of ${ }^{99 \mathrm{~m}} \mathrm{Tc}$-Cetuximab similar to the unspecific accumulation of the ${ }^{99 m}$ Tc-isotype antibody.

The anti-EGFR nanobody D10 was used in vivo for the first time. It could be shown that ${ }^{99 m}$ Tc-D10 represents a versatile tool for the specific detection of small EGFR overexpressing tumor lesions and the assessment of EGFR expression in tumors by in vivo SPECT. The identification of tumors at the earliest stage with ${ }^{99 \mathrm{~m}} \mathrm{Tc}$-D10 allowed the acquisition of high contrast images with a high tumor to background background ratio shortly after probe administration, i.e. allowing imaging at the same day. Therefore, ${ }^{99 \mathrm{~m}} \mathrm{Tc}-\mathrm{D} 10 \mathrm{might}$ be suitable in a clinical setting as non-invasive diagnostic tracer to not only detect small tumors but also to obtain information on the expression level of EGFR in heterogenic tumors at the time of diagnosis and during disease progression. 


\section{Outlook}

The anti-Kv10.1 nanobodies C4 and D9, that were generated in the course of this project, show very promising binding characteristics to this challenging target, but specific detection of the native ion channel remains to be validated. The successful application as in vivo tracers will depend on the tumor entity and the number of expressed potassium channels on the tumor cells as well as affinity of an engineered anti-Kv10.1 nanobody to its target protein. The application of the nanobodies as unmodified monomers might not lead to a successful tumor detection. However, genetically engineered bi- or trivalent nanobodies could facilitate this task.

The impact of the anti-Kv10.1 nanobodies on the potassium channel was not investigated yet. A possible impaired signaling due to the binding anti-Kv10.1 nanobodies C4 or D9 could refer to a therapeutic effect and an establishment of a specific Kv10.1 targeted therapy in future.

With the generation of the anti-Kv10.1 nanobodies C4 and D9, a versatile toolkit consisting of the two nanobodies with different fluorophores and enzymes for the detection of Kv10.1 are soon available for the integration in various laboratory methods like flow cytometry, immunofluorescence microscopy, histology, ELISA or Western blotting.

The anti-EGFR nanobody D10, which was applied in an in vivo SPECT approach for the first time, allowed a tumor visualization of small tumors with a high contrast shortly after administration. However, the high kidney uptake caused by the $6 \mathrm{xHis}$ tag remains problematic for further in vivo studies. Therefore, the nanobody needs to be reengineered and the use of different chelators might help to reduce the kidney uptake to continue the translation of D10 to larger tumor animal models. 


\section{Bibliography}

1. Stewart, B. W.; Wild, C.; International Agency for Research on Cancer; World Health Organization, World cancer report 2014. p xiv, 630 pages.

2. Krause, B. J.; Beyer, T.; Bockisch, A.; Delbeke, D.; Kotzerke, J.; Minkov, V.; Reiser, M.; Willich, N., [FDG-PET/CT in oncology. German Guideline]. Nuklearmedizin 2007, 46, 291-301.

3. Boellaard, R.; O'Doherty, M. J.; Weber, W. A.; Mottaghy, F. M.; Lonsdale, M. N.; Stroobants, S. G.; Oyen, W. J.; Kotzerke, J.; Hoekstra, O. S.; Pruim, J.; Marsden, P. K.; Tatsch, K.; Hoekstra, C. J.; Visser, E. P.; Arends, B.; Verzijlbergen, F. J.; Zijlstra, J. M.; Comans, E. F.; Lammertsma, A. A.; Paans, A. M.; Willemsen, A. T.; Beyer, T.; Bockisch, A.; Schaefer-Prokop, C.; Delbeke, D.; Baum, R. P.; Chiti, A.; Krause, B. J., FDG PET and $\mathrm{PET} / \mathrm{CT}$ : EANM procedure guidelines for tumour PET imaging: version 1.0. Eur J Nucl Med Mol Imaging 2010, 37, 181-200.

4. Voet, D.; Voet, J. G.; Pratt, C. W.; Beck-Sickinger, A., Lehrbuch der Biochemie. 2., aktualisierte und erw. Aufl. ed.; Wiley-VCH-Verl.: Weinheim, 2010; p XXVI, 1253 S.

5. D'Huyvetter, M.; Xavier, C.; Caveliers, V.; Lahoutte, T.; Muyldermans, S.; Devoogdt, N., Radiolabeled nanobodies as theranostic tools in targeted radionuclide therapy of cancer. Expert Opin Drug Deliv 2014, 11, 1939-1954.

6. Hassanzadeh-Ghassabeh, G.; Devoogdt, N.; De Pauw, P.; Vincke, C.; Muyldermans, S., Nanobodies and their potential applications. Nanomedicine (Lond) 2013, 8, 1013-1026.

7. Gainkam, L. O.; Huang, L.; Caveliers, V.; Keyaerts, M.; Hernot, S.; Vaneycken, I.; Vanhove, C.; Revets, H.; De Baetselier, P.; Lahoutte, T., Comparison of the biodistribution and tumor targeting of two $99 \mathrm{mTc}$-labeled anti-EGFR nanobodies in mice, using pinhole SPECT/micro-CT. J Nucl Med 2008, 49, 788-795.

8. Huang, L.; Gainkam, L. O.; Caveliers, V.; Vanhove, C.; Keyaerts, M.; De Baetselier, P.; Bossuyt, A.; Revets, H.; Lahoutte, T., SPECT imaging with 99mTc-labeled EGFR-specific nanobody for in vivo monitoring of EGFR expression. Mol Imaging Biol 2008, 10, 167175.

9. Pruszynski, M.; Koumarianou, E.; Vaidyanathan, G.; Revets, H.; Devoogdt, N.; Lahoutte, T.; Zalutsky, M. R., Targeting breast carcinoma with radioiodinated anti-HER2 Nanobody. Nucl Med Biol 2013, 40, 52-59.

10. Vaneycken, I.; Devoogdt, N.; Van Gassen, N.; Vincke, C.; Xavier, C.; Wernery, U.; Muyldermans, S.; Lahoutte, T.; Caveliers, V., Preclinical screening of anti-HER2 nanobodies for molecular imaging of breast cancer. FASEB J 2011, 25, 2433-2446.

11. Pardo, L. A.; Stuhmer, W., The roles of $\mathrm{K}(+)$ channels in cancer. Nat Rev Cancer 2014, 14, 39-48.

12. Wulff, H.; Castle, N. A.; Pardo, L. A., Voltage-gated potassium channels as therapeutic targets. Nat Rev Drug Discov 2009, 8, 982-1001.

13. Hemmerlein, B.; Weseloh, R. M.; Mello de Queiroz, F.; Knotgen, H.; Sanchez, A.; Rubio, M. E.; Martin, S.; Schliephacke, T.; Jenke, M.; Heinz Joachim, R.; Stuhmer, W.; Pardo, L. A., Overexpression of Eag1 potassium channels in clinical tumours. Mol Cancer 2006, 5, 41.

14. Martin, S.; Lino de Oliveira, C.; Mello de Queiroz, F.; Pardo, L. A.; Stuhmer, W.; Del Bel, E., Eag1 potassium channel immunohistochemistry in the CNS of adult rat and selected regions of human brain. Neuroscience 2008, 155, 833-844.

15. Martin, S.; Lino-de-Oliveira, C.; Joca, S. R.; Weffort de Oliveira, R.; Echeverry, M. B.; Da Silva, C. A.; Pardo, L.; Stuhmer, W.; Bel, E. D., Eag 1, Eag 2 and Kcnn3 gene brain expression of isolated reared rats. Genes Brain Behav 2010, 9, 918-924. 
16. Ludwig, J.; Weseloh, R.; Karschin, C.; Liu, Q.; Netzer, R.; Engeland, B.; Stansfeld, C.; Pongs, O., Cloning and functional expression of rat eag2, a new member of the ethera-go-go family of potassium channels and comparison of its distribution with that of eag1. Mol Cell Neurosci 2000, 16, 59-70.

17. Saganich, M. J.; Machado, E.; Rudy, B., Differential expression of genes encoding subthreshold-operating voltage-gated K+ channels in brain. J Neurosci 2001, 21, 46094624.

18. Meyer, R.; Schonherr, R.; Gavrilova-Ruch, O.; Wohlrab, W.; Heinemann, S. H., Identification of ether a go-go and calcium-activated potassium channels in human melanoma cells. J Membr Biol 1999, 171, 107-115.

19. Patt, S.; Preussat, K.; Beetz, C.; Kraft, R.; Schrey, M.; Kalff, R.; Schonherr, K.; Heinemann, S. H., Expression of ether a go-go potassium channels in human gliomas. Neurosci Lett 2004, 368, 249-253.

20. Mello de Queiroz, F.; Suarez-Kurtz, G.; Stuhmer, W.; Pardo, L. A., Ether a go-go potassium channel expression in soft tissue sarcoma patients. Mol Cancer 2006, 5, 42.

21. Wu, X.; Zhong, D.; Lin, B.; Zhai, W.; Ding, Z.; Wu, J., p38 MAPK regulates the expression of ether a go-go potassium channel in human osteosarcoma cells. Radiol Oncol 2013, 47, 42-49.

22. Lin, H.; Li, Z.; Chen, C.; Luo, X.; Xiao, J.; Dong, D.; Lu, Y.; Yang, B.; Wang, Z., Transcriptional and post-transcriptional mechanisms for oncogenic overexpression of ether a go-go K+ channel. PLoS One 2011, 6, e20362.

23. Downie, B. R.; Sanchez, A.; Knotgen, H.; Contreras-Jurado, C.; Gymnopoulos, M.; Weber, C.; Stuhmer, W.; Pardo, L. A., Eag1 expression interferes with hypoxia homeostasis and induces angiogenesis in tumors. J Biol Chem 2008, 283, 36234-36240.

24. Herrmann, S.; Ninkovic, M.; Kohl, T.; Lorinczi, E.; Pardo, L. A., Cortactin controls surface expression of the voltage-gated potassium channel K(V)10.1. J Biol Chem 2012, 287, 44151-44163.

25. Ninkovic, M.; Mitkovski, M.; Kohl, T.; Stuhmer, W.; Pardo, L. A., Physical and functional interaction of KV10.1 with Rabaptin-5 impacts ion channel trafficking. FEBS Lett 2012, 586, 3077-3084.

26. Hammadi, M.; Chopin, V.; Matifat, F.; Dhennin-Duthille, I.; Chasseraud, M.; Sevestre, H.; Ouadid-Ahidouch, H., Human ether a-gogo $\mathrm{K}(+)$ channel 1 (hEag1) regulates MDAMB-231 breast cancer cell migration through Orai1-dependent calcium entry. J Cell Physiol 2012, 227, 3837-3846.

27. Ousingsawat, J.; Spitzner, M.; Puntheeranurak, S.; Terracciano, L.; Tornillo, L.; Bubendorf, L.; Kunzelmann, K.; Schreiber, R., Expression of voltage-gated potassium channels in human and mouse colonic carcinoma. Clin Cancer Res 2007, 13, 824-831.

28. Asher, V.; Khan, R.; Warren, A.; Shaw, R.; Schalkwyk, G. V.; Bali, A.; Sowter, H. M., The Eag potassium channel as a new prognostic marker in ovarian cancer. Diagn Pathol 2010, 5, 78 .

29. Agarwal, J. R.; Griesinger, F.; Stuhmer, W.; Pardo, L. A., The potassium channel Ether a go-go is a novel prognostic factor with functional relevance in acute myeloid leukemia. Mol Cancer 2010, 9, 18.

30. Menendez, S. T.; Villaronga, M. A.; Rodrigo, J. P.; Alvarez-Teijeiro, S.; Garcia-Carracedo, D.; Urdinguio, R. G.; Fraga, M. F.; Pardo, L. A.; Viloria, C. G.; Suarez, C.; Garcia-Pedrero, J. M., Frequent aberrant expression of the human ether a go-go (hEAG1) potassium channel in head and neck cancer: pathobiological mechanisms and clinical implications. J Mol Med (Berl) 2012, 90, 1173-1184.

31. Toral, C.; Mendoza-Garrido, M. E.; Azorin, E.; Hernandez-Gallegos, E.; Gomora, J. C.; Delgadillo, D. M.; Solano-Agama, C.; Camacho, J., Effect of extracellular matrix on adhesion, viability, actin cytoskeleton and $\mathrm{K}+$ currents of cells expressing human ether a go-go channels. Life Sci 2007, 81, 255-265. 
32. Roy, J.; Vantol, B.; Cowley, E. A.; Blay, J.; Linsdell, P., Pharmacological separation of hEAG and hERG K+ channel function in the human mammary carcinoma cell line MCF7. Oncol Rep 2008, 19, 1511-1516.

33. Prenzel, N.; Fischer, O. M.; Streit, S.; Hart, S.; Ullrich, A., The epidermal growth factor receptor family as a central element for cellular signal transduction and diversification. Endocr Relat Cancer 2001, 8, 11-31.

34. Oda, K.; Matsuoka, Y.; Funahashi, A.; Kitano, H., A comprehensive pathway map of epidermal growth factor receptor signaling. Mol Syst Biol 2005, 1, 20050010.

35. Jost, M.; Kari, C.; Rodeck, U., The EGF receptor - an essential regulator of multiple epidermal functions. Eur J Dermatol 2000, 10, 505-510.

36. Alberts, B., Molekularbiologie der Zelle. In 5. Aufl. ed.; Wiley-VCH: Weinheim, 2011.

37. Downward, J.; Parker, P.; Waterfield, M. D., Autophosphorylation sites on the epidermal growth factor receptor. Nature 1984, 311, 483-485.

38. Klein, S.; Levitzki, A., Targeting the EGFR and the PKB pathway in cancer. Curr Opin Cell Biol 2009, 21, 185-193.

39. Tebbutt, N.; Pedersen, M. W.; Johns, T. G., Targeting the ERBB family in cancer: couples therapy. Nat Rev Cancer 2013, 13, 663-673.

40. Zhang, X.; Chang, A., Molecular predictors of EGFR-TKI sensitivity in advanced nonsmall cell lung cancer. Int J Med Sci 2008, 5, 209-217.

41. Liao, B. C.; Lin, C. C.; Yang, J. C., First-line management of EGFR-mutated advanced lung adenocarcinoma: recent developments. Drugs 2013, 73, 357-369.

42. Bria, E.; Milella, M.; Cuppone, F.; Novello, S.; Ceribelli, A.; Vaccaro, V.; Sperduti, I.; Gelibter, A.; Scagliotti, G. V.; Cognetti, F.; Giannarelli, D., Outcome of advanced NSCLC patients harboring sensitizing EGFR mutations randomized to EGFR tyrosine kinase inhibitors or chemotherapy as first-line treatment: a meta-analysis. Ann Oncol 2011, 22, 2277-2285.

43. Wheeler, D. L.; Dunn, E. F.; Harari, P. M., Understanding resistance to EGFR inhibitorsimpact on future treatment strategies. Nat Rev Clin Oncol 2010, 7, 493-507.

44. Geuna, E.; Montemurro, F.; Aglietta, M.; Valabrega, G., Potential of afatinib in the treatment of patients with HER2-positive breast cancer. Breast Cancer (Dove Med Press) 2012, 4, 131-137.

45. Schmitz, K. R.; Ferguson, K. M., Interaction of antibodies with ErbB receptor extracellular regions. Exp Cell Res 2009, 315, 659-670.

46. Caliceti, P.; Veronese, F. M., Pharmacokinetic and biodistribution properties of poly(ethylene glycol)-protein conjugates. Adv Drug Deliv Rev 2003, 55, 1261-1277.

47. Kijanka, M.; Dorresteijn, B.; Oliveira, S.; van Bergen en Henegouwen, P. M., Nanobodybased cancer therapy of solid tumors. Nanomedicine (Lond) 2015, 10, 161-174.

48. Vaneycken, I.; Govaert, J.; Vincke, C.; Caveliers, V.; Lahoutte, T.; De Baetselier, P.; Raes, G.; Bossuyt, A.; Muyldermans, S.; Devoogdt, N., In vitro analysis and in vivo tumor targeting of a humanized, grafted nanobody in mice using pinhole SPECT/micro-CT. J Nucl Med 2010, 51, 1099-1106.

49. Vincke, C.; Loris, R.; Saerens, D.; Martinez-Rodriguez, S.; Muyldermans, S.; Conrath, K., General strategy to humanize a camelid single-domain antibody and identification of a universal humanized nanobody scaffold. J Biol Chem 2009, 284, 3273-3284.

50. Siontorou, C. G., Nanobodies as novel agents for disease diagnosis and therapy. Int J Nanomedicine 2013, 8, 4215-4227.

51. Evazalipour, M.; D'Huyvetter, M.; Tehrani, B. S.; Abolhassani, M.; Omidfar, K.; Abdoli, S.; Arezumand, R.; Morovvati, H.; Lahoutte, T.; Muyldermans, S.; Devoogdt, N., Generation and characterization of nanobodies targeting PSMA for molecular imaging of prostate cancer. Contrast Media Mol Imaging 2014, 9, 211-220. 
52. Xavier, C.; Vaneycken, I.; D'Huyvetter, M.; Heemskerk, J.; Keyaerts, M.; Vincke, C.; Devoogdt, N.; Muyldermans, S.; Lahoutte, T.; Caveliers, V., Synthesis, preclinical validation, dosimetry, and toxicity of 68Ga-NOTA-anti-HER2 Nanobodies for iPET imaging of HER2 receptor expression in cancer. J Nucl Med 2013, 54, 776-784.

53. Gainkam, L. O.; Caveliers, V.; Devoogdt, N.; Vanhove, C.; Xavier, C.; Boerman, O.; Muyldermans, S.; Bossuyt, A.; Lahoutte, T., Localization, mechanism and reduction of renal retention of technetium-99m labeled epidermal growth factor receptor-specific nanobody in mice. Contrast Media Mol Imaging 2011, 6, 85-92.

54. Holz, J. B., The TITAN trial--assessing the efficacy and safety of an anti-von Willebrand factor Nanobody in patients with acquired thrombotic thrombocytopenic purpura. Transfus Apher Sci 2012, 46, 343-346.

55. Ablynx Clinical Trials. http://www.ablynx.com/rd-portfolio/clinical-programmes/ (27.8.),

56. Sukhanova, A.; Even-Desrumeaux, K.; Kisserli, A.; Tabary, T.; Reveil, B.; Millot, J. M.; Chames, P.; Baty, D.; Artemyev, M.; Oleinikov, V.; Pluot, M.; Cohen, J. H.; Nabiev, I., Oriented conjugates of single-domain antibodies and quantum dots: toward a new generation of ultrasmall diagnostic nanoprobes. Nanomedicine 2012, 8, 516-525.

57. Committee, N. R. C. U., Medical Isotope Production Without Highly Enriched Uranium. 2014/07/11 ed.; National Academy of Sciences: 2009.

58. Amato, I., NUCLEAR MEDICINE'S CONUNDRUM. Chemical \& Engineering News Archive 2009, 87, 58-64.

59. Schwochau, K., Technetium Radiopharmaceuticals-Fundamentals, Synthesis, Structure, and Development. Angewandte Chemie International Edition in English 1994, 33, 2258-2267.

60. Saha, G. B., Fundamentals of nuclear pharmacy. 6. ed.; Springer: New York, NY '[u.a.]\%o, 2010; p XVIII, $409 \mathrm{~S}$.

61. Liu, S.; Chakraborty, S., 99mTc-centered one-pot synthesis for preparation of 99mTc radiotracers. Dalton Trans 2011, 40, 6077-6086.

62. Liu, S., Bifunctional coupling agents for radiolabeling of biomolecules and targetspecific delivery of metallic radionuclides. Adv Drug Deliv Rev 2008, 60, 1347-1370.

63. Liu, S., The role of coordination chemistry in the development of target-specific radiopharmaceuticals. Chemical Society Reviews 2004, 33, 445-461.

64. Vallabhajosula, S., Molecular imaging : radiopharmaceuticals for PET and SPECT. Springer: Dordrecht ; London, 2009; p xix, 371 p.

65. Eberle, A. N.; Mild, G., Receptor-mediated tumor targeting with radiopeptides. Part 1. General principles and methods. J Recept Signal Transduct Res 2009, 29, 1-37.

66. Krause, W.; Alberto, R., Radiopharmaceuticals - from diagnostics to therapeutics. Springer: Berlin ^[u.a.]\%o, 2005; p VIII, 222 S.

67. Meszaros, L. K.; Dose, A.; Biagini, S. C.; Blower, P. J., Synthesis and evaluation of analogues of HYNIC as bifunctional chelators for technetium. Dalton Trans 2011, 40, 6260-6267.

68. Meszaros, L. K.; Dose, A.; Biagini, S. C. G.; Blower, P. J., Hydrazinonicotinic acid (HYNIC) - Coordination chemistry and applications in radiopharmaceutical chemistry. Inorganica Chimica Acta 2010, 363, 1059-1069.

69. Waibel, R.; Alberto, R.; Willuda, J.; Finnern, R.; Schibli, R.; Stichelberger, A.; Egli, A.; Abram, U.; Mach, J. P.; Pluckthun, A.; Schubiger, P. A., Stable one-step technetium$99 \mathrm{~m}$ labeling of His-tagged recombinant proteins with a novel Tc(I)-carbonyl complex. Nat Biotechnol 1999, 17, 897-901.

70. Egli, A.; Alberto, R.; Tannahill, L.; Schibli, R.; Abram, U.; Schaffland, A.; Waibel, R.; Tourwe, D.; Jeannin, L.; Iterbeke, K.; Schubiger, P. A., Organometallic 99mTc-aquaion labels peptide to an unprecedented high specific activity. J Nucl Med 1999, 40, 19131917. 
71. Schibli, R.; Katti, K. V.; Higginbotham, C.; Volkert, W. A.; Alberto, R., In vitro and in vivo evaluation of bidentate, water-soluble phosphine ligands as anchor groups for the organometallic fac-[99mTc(CO)3]+-core. Nucl Med Biol 1999, 26, 711-716.

72. Schibli, R.; La Bella, R.; Alberto, R.; Garcia-Garayoa, E.; Ortner, K.; Abram, U.; Schubiger, $P$. A., Influence of the denticity of ligand systems on the in vitro and in vivo behavior of $(99 \mathrm{~m}) \mathrm{Tc}(\mathrm{I})$-tricarbonyl complexes: a hint for the future functionalization of biomolecules. Bioconjug Chem 2000, 11, 345-351.

73. Seo, Y.; Mari, C.; Hasegawa, B. H., Technological development and advances in singlephoton emission computed tomography/computed tomography. Semin Nucl Med 2008, 38, 177-198.

74. Granov, A. M.; Tiutin, L. A.; Schwarz, T.; Ohio Library and Information Network., Positron emission tomography. In Springer: Berlin ; New York, 2013; p 1 online resource.

75. Schering Stiftung, Ernst Schering Foundation symposium proceedings. In Springer: Berlin; Heidelberg, pp Online-Ressource.

76. Hutton, B. F.; Hudson, H. M.; Beekman, F. J., A clinical perspective of accelerated statistical reconstruction. Eur J Nucl Med 1997, 24, 797-808.

77. Hudson, H. M.; Larkin, R. S., Accelerated image reconstruction using ordered subsets of projection data. IEEE Trans Med Imaging 1994, 13, 601-609.

78. Healthcare, $\quad S$ Biograph TruePoint PET/CT. http://www.healthcare.siemens.de/molecular-imaging/pet-ct/biograph-truepointpetct (29.08.),

79. Mediso nanoScan PET/CT. http://www.mediso.de/nanoscan-pet-ct.htnl (29.08.),

80. HZDI https://www.hzdr.de/FWS/FWSF/messtechnik/pet/petschema_en.gif (29.08.),

81. Schober, O.; Riemann, B.; Ohio Library and Information Network., Molecular imaging in oncology. In Recent results in cancer research v.187., Springer: Berlin ; London, 2013; p 1 online resource.

82. Beekman, F.; van der Have, F., The pinhole: gateway to ultra-high-resolution threedimensional radionuclide imaging. European Journal of Nuclear Medicine and Molecular Imaging 2007, 34, 151-161.

83. Mettler, F. A., Jr.; Huda, W.; Yoshizumi, T. T.; Mahesh, M., Effective doses in radiology and diagnostic nuclear medicine: a catalog. Radiology 2008, 248, 254-263.

84. DePuey, E. G. http://www.imagewisely.org/imaging-modalities/nuclearmedicine/articles/myocardial-perfusion-spect (29.08.),

85. Nuyts, J.; Vunckx, K.; Defrise, M.; Vanhove, C., Small animal imaging with multi-pinhole SPECT. Methods 2009, 48, 83-91.

86. Ivashchenko, O.; van der Have, F.; Goorden, M. C.; Ramakers, R. M.; Beekman, F. J., Ultra-high-sensitivity submillimeter mouse SPECT. J Nucl Med 2015, 56, 470-475.

87. Morton, C. L.; Houghton, P. J., Establishment of human tumor xenografts in immunodeficient mice. Nat Protoc 2007, 2, 247-250.

88. Flanagan, S. P., 'Nude', a new hairless gene with pleiotropic effects in the mouse. Genet Res 1966, 8, 295-309.

89. Murphy, K. P.; Travers, P.; Walport, M. J.; Janeway, C., Janeway Immunologie. 7. Aufl. ed.; Spektrum Akad. Verl.: Heidelberg, 2009; p XXVI, 1093 S.

90. Kubota, T., Metastatic models of human cancer xenografted in the nude mouse: the importance of orthotopic transplantation. J Cell Biochem 1994, 56, 4-8.

91. Kuo, T. H.; Kubota, T.; Watanabe, M.; Furukawa, T.; Teramoto, T.; Ishibiki, K.; Kitajima, M.; Hoffman, R. M., Early resection of primary orthotopically-growing human colon tumor in nude mouse prevents liver metastasis: further evidence for patient-like hematogenous metastatic route. Anticancer Res 1993, 13, 293-297. 
92. Kuo, T. H.; Kubota, T.; Watanabe, M.; Furukawa, T.; Kase, S.; Tanino, H.; Saikawa, Y.; Ishibiki, K.; Kitajima, M.; Hoffman, R. M., Site-specific chemosensitivity of human smallcell lung carcinoma growing orthotopically compared to subcutaneously in SCID mice: the importance of orthotopic models to obtain relevant drug evaluation data. Anticancer Res 1993, 13, 627-630.

93. Kuo, T. H.; Kubota, T.; Watanabe, M.; Furukawa, T.; Teramoto, T.; Ishibiki, K.; Kitajima, M.; Moossa, A. R.; Penman, S.; Hoffman, R. M., Liver colonization competence governs colon cancer metastasis. Proc Natl Acad Sci U S A 1995, 92, 12085-12089.

94. Reilly, R. M.; Kiarash, R.; Sandhu, J.; Lee, Y. W.; Cameron, R. G.; Hendler, A.; Vallis, K.; Gariepy, J., A comparison of EGF and MAb 528 labeled with 111 In for imaging human breast cancer. J Nucl Med 2000, 41, 903-911.

95. Haigler, H.; Ash, J. F.; Singer, S. J.; Cohen, S., Visualization by fluorescence of the binding and internalization of epidermal growth factor in human carcinoma cells A431. Proc Natl Acad Sci U S A 1978, 75, 3317-3321.

96. Nevoltris, D.; Lombard, B.; Dupuis, E.; Mathis, G.; Chames, P.; Baty, D., Conformational nanobodies reveal tethered epidermal growth factor receptor involved in EGFR/ErbB2 predimers. ACS Nano 2015, 9, 1388-1399.

97. Chames, P., Antibody engineering : methods and protocols. 2nd ed.; Humana Press: New York, 2012; p xvi, 734 p.

98. Muyldermans, S., Nanobodies: natural single-domain antibodies. Annu Rev Biochem 2013, 82, 775-797.

99. Rucker, J.; Payne, R.; Baxter, S.; Tucker, D.; Willis, S. H.; Doranz, B. J., Generation of Antibodies Against Ion Channels Using Virus-Like Particles. Biophysical Journal 2011, 100, 91a.

100. Wilkinson, T. C.; Gardener, M. J.; Williams, W. A., Discovery of functional antibodies targeting ion channels. J Biomol Screen 2015, 20, 454-467.

101. Conn, P. M., Essential ion channel methods. Elsevier/Academic Press: Amsterdam, 2010; p xxi, 606 p.

102. Ablynx Nanobodies against difficult targets - Tackling ion channels. http://www.ablynx.com/uploads/data/files/collaborations-in-ion-channel-drugdiscovery_19-june-2014.pdf (25.09.),

103. Fleetwood, F.; Klint, S.; Hanze, M.; Gunneriusson, E.; Frejd, F. Y.; Stahl, S.; Lofblom, J., Simultaneous targeting of two ligand-binding sites on VEGFR2 using biparatopic Affibody molecules results in dramatically improved affinity. Sci Rep 2014, 4, 7518.

104. Rudnick, S. I.; Adams, G. P., Affinity and avidity in antibody-based tumor targeting. Cancer Biother Radiopharm 2009, 24, 155-161.

105. Hartung, F.; Stuhmer, W.; Pardo, L. A., Tumor cell-selective apoptosis induction through targeting of K(V)10.1 via bifunctional TRAIL antibody. Mol Cancer 2011, 10, 109.

106. Pardo, L. A.; Stuhmer, W., Eag1: an emerging oncological target. Cancer Res 2008, 68, 1611-1613.

107. Rodriguez-Rasgado, J. A.; Acuna-Macias, I.; Camacho, J., Eag1 channels as potential cancer biomarkers. Sensors (Basel) 2012, 12, 5986-5995.

108. Restrepo-Angulo, I.; Sanchez-Torres, C.; Camacho, J., Human EAG1 potassium channels in the epithelial-to-mesenchymal transition in lung cancer cells. Anticancer Res 2011, 31, 1265-1270.

109. Rousserie, G.; Grinevich, R.; Brazhnik, K.; Even-Desrumeaux, K.; Reveil, B.; Tabary, T.; Chames, P.; Baty, D.; Cohen, J. H.; Nabiev, I.; Sukhanova, A., Detection of carcinoembryonic antigen using single-domain or full-size antibodies stained with quantum dot conjugates. Anal Biochem 2015, 478, 26-32.

110. Turini, M.; Chames, P.; Bruhns, P.; Baty, D.; Kerfelec, B., A FcgammaRIII-engaging bispecific antibody expands the range of HER2-expressing breast tumors eligible to antibody therapy. Oncotarget 2014, 5, 5304-5319. 
111. Majidi, J.; Barar, J.; Baradaran, B.; Abdolalizadeh, J.; Omidi, Y., Target therapy of cancer: implementation of monoclonal antibodies and nanobodies. Hum Antibodies 2009, 18, 81-100.

112. Sihver, W.; Pietzsch, J.; Krause, M.; Baumann, M.; Steinbach, J.; Pietzsch, H. J., Radiolabeled Cetuximab Conjugates for EGFR Targeted Cancer Diagnostics and Therapy. Pharmaceuticals (Basel) 2014, 7, 311-338.

113. Tijink, B. M.; Laeremans, T.; Budde, M.; Stigter-van Walsum, M.; Dreier, T.; de Haard, H. J.; Leemans, C. R.; van Dongen, G. A., Improved tumor targeting of anti-epidermal growth factor receptor Nanobodies through albumin binding: taking advantage of modular Nanobody technology. Mol Cancer Ther 2008, 7, 2288-2297.

114. Greish, K., Enhanced permeability and retention of macromolecular drugs in solid tumors: a royal gate for targeted anticancer nanomedicines. J Drug Target 2007, 15, 457-464.

115. Maeda, H.; Fang, J.; Inutsuka, T.; Kitamoto, Y., Vascular permeability enhancement in solid tumor: various factors, mechanisms involved and its implications. Int Immunopharmacol 2003, 3, 319-328.

116. Maeda, H.; Wu, J.; Sawa, T.; Matsumura, Y.; Hori, K., Tumor vascular permeability and the EPR effect in macromolecular therapeutics: a review. J Control Release 2000, 65, 271-284.

117. Haag, R.; Kratz, F., Polymer therapeutics: concepts and applications. Angew Chem Int Ed Eng/ 2006, 45, 1198-1215.

118. Lippert, H., Lehrbuch Anatomie 184 Tabellen. 5., völlig überarb. Aufl. ed.; Urban und Fischer: München ^[u.a.]\%o, 2000; p XII, 803 S.

119. Choi, I.; Lee, S.; Hong, Y. K., The new era of the lymphatic system: no longer secondary to the blood vascular system. Cold Spring Harb Perspect Med 2012, 2, a006445.

120. Shayan, R.; Achen, M. G.; Stacker, S. A., Lymphatic vessels in cancer metastasis: bridging the gaps. Carcinogenesis 2006, 27, 1729-1738.

121. Rahbar, H.; Partridge, S. C.; Javid, S. H.; Lehman, C. D., Imaging axillary lymph nodes in patients with newly diagnosed breast cancer. Curr Probl Diagn Radiol 2012, 41, 149158.

122. Scully, O. J.; Bay, B. H.; Yip, G.; Yu, Y., Breast cancer metastasis. Cancer Genomics Proteomics 2012, 9, 311-320.

123. Robbins, A. R.; Oliver, C.; Bateman, J. L.; Krag, S. S.; Galloway, C. J.; Mellman, I., A single mutation in Chinese hamster ovary cells impairs both Golgi and endosomal functions. $J$ Cell Biol 1984, 99, 1296-1308.

124. Tesar, D. B.; Bjorkman, P. J., An intracellular traffic jam: Fc receptor-mediated transport of immunoglobulin G. Curr Opin Struct Biol 2010, 20, 226-233.

125. Anderson, C. J.; Bulte, J. W.; Chen, K.; Chen, X.; Khaw, B. A.; Shokeen, M.; Wooley, K. L.; VanBrocklin, H. F., Design of targeted cardiovascular molecular imaging probes. J Nucl Med 2010, 51 Suppl 1, 3S-17S.

126. Schoffelen, R.; Sharkey, R. M.; Goldenberg, D. M.; Franssen, G.; McBride, W. J.; Rossi, E. A.; Chang, C. H.; Laverman, P.; Disselhorst, J. A.; Eek, A.; van der Graaf, W. T.; Oyen, W. J.; Boerman, O. C., Pretargeted immuno-positron emission tomography imaging of carcinoembryonic antigen-expressing tumors with a bispecific antibody and a 68Gaand $18 \mathrm{~F}$-labeled hapten peptide in mice with human tumor xenografts. Mol Cancer Ther 2010, 9, 1019-1027.

127. Sharkey, R. M.; Rossi, E. A.; McBride, W. J.; Chang, C. H.; Goldenberg, D. M., Recombinant bispecific monoclonal antibodies prepared by the dock-and-lock strategy for pretargeted radioimmunotherapy. Semin Nucl Med 2010, 40, 190-203.

128. Tekabe, Y.; Einstein, A. J.; Johnson, L. L.; Khaw, B. A., Targeting very small model lesions pretargeted with bispecific antibody with $99 \mathrm{mTc}$-labeled high-specific radioactivity polymers. Nucl Med Commun 2010, 31, 320-327. 
129. Karacay, H.; Sharkey, R. M.; McBride, W. J.; Rossi, E. A.; Chang, C. H.; Goldenberg, D. M., Optimization of hapten-peptide labeling for pretargeted immunoPET of bispecific antibody using generator-produced 68Ga. J Nucl Med 2011, 52, 555-559.

130. Oliveira, S.; van Dongen, G. A.; Stigter-van Walsum, M.; Roovers, R. C.; Stam, J. C.; Mali, W.; van Diest, P. J.; van Bergen en Henegouwen, P. M., Rapid visualization of human tumor xenografts through optical imaging with a near-infrared fluorescent antiepidermal growth factor receptor nanobody. Mol Imaging 2012, 11, 33-46.

131. Holash, J.; Maisonpierre, P. C.; Compton, D.; Boland, P.; Alexander, C. R.; Zagzag, D.; Yancopoulos, G. D.; Wiegand, S. J., Vessel cooption, regression, and growth in tumors mediated by angiopoietins and VEGF. Science 1999, 284, 1994-1998.

132. Holash, J.; Wiegand, S. J.; Yancopoulos, G. D., New model of tumor angiogenesis: dynamic balance between vessel regression and growth mediated by angiopoietins and VEGF. Oncogene 1999, 18, 5356-5362.

133. Medri, L.; Nanni, O.; Volpi, A.; Scarpi, E.; Dubini, A.; Riccobon, A.; Becciolini, A.; Bianchi, S.; Amadori, D., Tumor microvessel density and prognosis in node-negative breast cancer. Int J Cancer 2000, 89, 74-80.

134. Di Benedetto, M.; Starzec, A.; Vassy, R.; Perret, G. Y.; Crepin, M.; Kraemer, M., Inhibition of epidermoid carcinoma A431 cell growth and angiogenesis in nude mice by early and late treatment with a novel dextran derivative. Br J Cancer 2003, 88, 19871994.

135. Ullrich, R. T.; Jikeli, J. F.; Diedenhofen, M.; Bohm-Sturm, P.; Unruh, M.; Vollmar, S.; Hoehn, M., In-vivo visualization of tumor microvessel density and response to antiangiogenic treatment by high resolution MRI in mice. PLoS One 2011, 6, e19592.

136. Ehling, J.; Theek, B.; Gremse, F.; Baetke, S.; Mockel, D.; Maynard, J.; Ricketts, S. A.; Grull, H.; Neeman, M.; Knuechel, R.; Lederle, W.; Kiessling, F.; Lammers, T., Micro-CT imaging of tumor angiogenesis: quantitative measures describing micromorphology and vascularization. Am J Pathol 2014, 184, 431-441.

137. Shipitsin, M.; Campbell, L. L.; Argani, P.; Weremowicz, S.; Bloushtain-Qimron, N.; Yao, J.; Nikolskaya, T.; Serebryiskaya, T.; Beroukhim, R.; Hu, M.; Halushka, M. K.; Sukumar, S.; Parker, L. M.; Anderson, K. S.; Harris, L. N.; Garber, J. E.; Richardson, A. L.; Schnitt, S. J.; Nikolsky, Y.; Gelman, R. S.; Polyak, K., Molecular definition of breast tumor heterogeneity. Cancer Cell 2007, 11, 259-273.

138. Marusyk, A.; Polyak, K., Tumor heterogeneity: causes and consequences. Biochim Biophys Acta 2010, 1805, 105-117.

139. Jamal-Hanjani, M.; Quezada, S. A.; Larkin, J.; Swanton, C., Translational implications of tumor heterogeneity. Clin Cancer Res 2015, 21, 1258-1266.

140. Patel, D.; Lahiji, A.; Patel, S.; Franklin, M.; Jimenez, X.; Hicklin, D. J.; Kang, X., Monoclonal antibody cetuximab binds to and down-regulates constitutively activated epidermal growth factor receptor vIII on the cell surface. Anticancer Res 2007, 27, 3355-3366.

141. Vaneycken, I.; D'Huyvetter, M.; Hernot, S.; De Vos, J.; Xavier, C.; Devoogdt, N.; Caveliers, V.; Lahoutte, T., Immuno-imaging using nanobodies. Curr Opin Biotechnol 2011, 22, 877-881.

142. Behr, T. M.; Becker, W. S.; Sharkey, R. M.; Juweid, M. E.; Dunn, R. M.; Bair, H. J.; Wolf, F. G.; Goldenberg, D. M., Reduction of renal uptake of monoclonal antibody fragments by amino acid infusion. J Nucl Med 1996, 37, 829-833.

143. Behr, T. M.; Sharkey, R. M.; Juweid, M. E.; Blumenthal, R. D.; Dunn, R. M.; Griffiths, G. L.; Bair, H. J.; Wolf, F. G.; Becker, W. S.; Goldenberg, D. M., Reduction of the renal uptake of radiolabeled monoclonal antibody fragments by cationic amino acids and their derivatives. Cancer Res 1995, 55, 3825-3834.

144. Behr, T. M.; Goldenberg, D. M.; Becker, W., Reducing the renal uptake of radiolabeled antibody fragments and peptides for diagnosis and therapy: present status, future prospects and limitations. Eur J Nucl Med 1998, 25, 201-212. 
145. Devoogdt, N.; Xavier, C.; Hernot, S.; Vaneycken, I.; D'Huyvetter, M.; De Vos, J.; Massa, S.; De Baetselier, P.; Caveliers, V.; Lahoutte, T., Molecular imaging using Nanobodies: a case study. Methods Mol Biol 2012, 911, 559-567.

146. Orlova, A.; Tolmachev, V.; Pehrson, R.; Lindborg, M.; Tran, T.; Sandström, M.; Nilsson, F. Y.; Wennborg, A.; Abrahmsén, L.; Feldwisch, J., Synthetic Affibody Molecules: A Novel Class of Affinity Ligands for Molecular Imaging of HER2-Expressing Malignant Tumors. Cancer Research 2007, 67, 2178-2186.

147. van der Meel, R.; Oliveira, S.; Altintas, I.; Haselberg, R.; van der Veeken, J.; Roovers, R. C.; van Bergen en Henegouwen, P. M.; Storm, G.; Hennink, W. E.; Schiffelers, R. M.; Kok, R. J., Tumor-targeted Nanobullets: Anti-EGFR nanobody-liposomes loaded with anti-IGF-1R kinase inhibitor for cancer treatment. J Control Release 2012, 159, 281289.

148. Olson, G. E.; Winfrey, V. P.; Hill, K. E.; Burk, R. F., Megalin mediates selenoprotein P uptake by kidney proximal tubule epithelial cells. J Biol Chem 2008, 283, 6854-6860.

149. Motoyoshi, Y.; Matsusaka, T.; Saito, A.; Pastan, I.; Willnow, T. E.; Mizutani, S.; Ichikawa, I., Megalin contributes to the early injury of proximal tubule cells during nonselective proteinuria. Kidney Int 2008, 74, 1262-1269.

150. Hall, E. J.; Giaccia, A. J., Radiobiology for the radiologist. 6. ed.; Lippincott Williams \& Wilkins: Philadelphia, Pa. ^[u.a.]\%o, 2006; p IX, 546 S.

151. Chatalic, K. L.; Veldhoven-Zweistra, J.; Bolkestein, M.; Hoeben, S.; Koning, G. A.; Boerman, O. C.; de Jong, M.; van Weerden, W. M., A novel 111In-labeled anti-PSMA nanobody for targeted SPECT/CT imaging of prostate cancer. J Nucl Med 2015.

152. Bellucci, J. J.; Bhattacharyya, J.; Chilkoti, A., A noncanonical function of sortase enables site-specific conjugation of small molecules to lysine residues in proteins. Angew Chem Int Ed Engl 2015, 54, 441-445.

153. Bellucci, J. J.; Amiram, M.; Bhattacharyya, J.; McCafferty, D.; Chilkoti, A., Three-in-one chromatography-free purification, tag removal, and site-specific modification of recombinant fusion proteins using sortase $A$ and elastin-like polypeptides. Angew Chem Int Ed Engl 2013, 52, 3703-3708.

154. Strijbis, K.; Spooner, E.; Ploegh, H. L., Protein ligation in living cells using sortase. Traffic 2012, 13, 780-789. 


\section{Curriculum vitae}

Personal details

Name

Surname

Date

Place of birth

Nationality

Adress

Thomas

Krüwel

March 4 ${ }^{\text {th }}, 1984$

Hannover, Germany

German

Breite Str. 13A, 37077 Göttingen

\section{Career summary}

10/2011-present

Research assistant, Department of Interventional and Diagnostic Radiology, University Medical Center Göttingen, Göttingen, Germany

01/2010-09/2011 Research assistant, Department of Pharmaco- and Toxicogenomics, Hannover Medical School, Hannover, Germany

University authorizations

01/2014-present Radiation protection commissioner (Strahlenschutzbeauftragter; RöV), University Medical Center Göttingen, Göttingen, Germany

08/2012-present Radiation protection commissioner (Strahlenschutzbeauftragter, StrlSchV), University Medical Center Göttingen, Göttingen, Germany

Education

10/2011-present $\quad$ PhD program "Molecular Medicine", University Medical Center Göttingen, Göttingen, Germany

01/2010-09/2011 PhD student in Biology, Gottfried Wilhelm Leibniz University Hannover, Hannover, Germany

10/2007-09/2009 Master of Science, Life Science, Gottfried Wilhelm Leibniz University Hannover, Hannover, Germany Thesis: "Charakterisierung experimenteller c-ABL/c-Src duale 
Kinase Hemmstoffe in der Therapie des nicht-Kleinzelligen Adenokarzinoms der Lunge" Grade: summa cum laude

10/2004-07/2007 Bachelor of Science, Life Science, Gottfried Wilhelm Leibniz University Hannover, Hannover, Germany Thesis: "Vergleich und Charakterisierung von binären Vektoren mittels bioinformatischer Methoden" Grade: magna cum laude

11/2003-08/2004 Civil service at "Altenpflegeheim am Listholze", Deutsches Rotes Kreuz, Hannover, Germany

$06 / 2003$

Abitur, Albert-Einstein-Schule, Laatzen, Germany

Presentations at scientific meetings

$09 / 2015$

$03 / 2015$

$09 / 2013$

$05 / 2013$

Poster presentation "Visualization of EGFR expressing mammary carcinomas in mice by SPECT applying ${ }^{99 \mathrm{~m}} \mathrm{Tc}(\mathrm{CO})_{3^{-}}$ labeled single domain antibodies" at the "World Molecular Imaging Congress", Honolulu, HI, USA

Poster presentation "Preclinical evaluation of single-photon emission computed tomography (SPECT) using a EGFRtargeting single domain antibody (sdAb) in an orthotopic mammary carcinoma mouse model" at the "European Molecular Imaging Meeting", Tübingen, Germany Poster presentation "Preclinical evaluation of single-photon computed tomography (SPECT) targeting EGFR in orthotopic breast cancer tumor models" at the "World Molecular Imaging Congress", Savannah, GA, USA

Oral presentation "Application of single-photon computed tomography (SPECT) targeting EGFR in orthotopic glioma mouse model" at the "European Molecular Imaging Meeting", Torino, Italy

$03 / 2013$

Oral presentation "SPECT-based in vivo imaging of the ether-àgo-go voltage-gated potassium channel (Eag1; Kv10.1)" at the " $2^{\text {nd }}$ Ringberg Conference on Ion Channels and Cancer", Kreuth, Germany 